\title{
BLUETOOTH RECEIVER DESIGN BASED ON
} LAURENT'S DECOMPOSITION

\author{
by
}

NOHA IBRAHIM

B.Eng, American University of Beirut, 2003

\begin{abstract}
A THESIS SUBMITTED IN PARTIAL FULFILLMENT OF THE REQUIREMENTS FOR THE DEGREE OF

MASTER OF APPLIED SCIENCE

in
\end{abstract}

THE FACULTY OF GRADUATE STUDIES

(Electrical and Computer Engineering)

December, 2005

(C) Noha Ibrahim, 2005 


\section{Abstract}

Bluetooth is a widely used communication standard for wireless personal area networks (WPAN). The Bluetooth transmit signal is Gaussian frequency shift keying (GFSK) modulated. GFSK belongs to the family of continuous-phase modulation (CPM) signals, which achieve a good trade-off between power and bandwidth efficiency and, due to constant envelope modulation, allow for low-complexity transmitter implementation. Bluetooth devices often employ a simple discriminator receiver, which is highly suboptimum in terms of power efficiency compared to the optimum receiver. Other approaches proposed in the literature consider trellis-based detection using the Viterbi or forward-backward algorithm. These schemes achieve significant performance improvements over discriminator detectors while entailing a considerably higher computational complexity. The main challenges faced when designing a Bluetooth sequence detector is the varying modulation index, which results in a varying trellis structure, and the time-variant channel phase, making coherent detection which assumes perfect channel phase estimation an almost impossible task.

In this research work, we present a receiver design for Bluetooth transmission based on Laurent's decomposition of the Bluetooth transmit signal. The main features of this receiver are its low-complexity compared to alternative solutions, its excellent performance close to the theoretical limit, and its high robustness against frequency offsets, phase noise, and modulation index variations, which are characteristic for lowcost Bluetooth devices. In particular, we show that the devised noncoherent decision- 
feedback equalization receiver achieves a similar performance as a recently proposed 2-state noncoherent sequence detector, while it is advantageous in terms of complexity. The new receiver design is therefore highly attractive for a practical implementation. 


\section{Contents}

Abstract

ii

Contents

iv

List of Tables

vii

List of Figures

viii

Glossary

xii

Acknowledgements

xvii

1 Introduction 1

1.1 The Evolution of the Bluetooth Technology . . . . . . . . . . . . 2

1.2 Challenges and Motivation . . . . . . . . . . . . . . . . 4

1.3 Contributions ....................... 6

1.4 Thesis Outline ......................... 7

2 Background 9

2.1 The Bluetooth System . . . . . . . . . . . . . . . 9

2.1.1 Radio Front End . . . . . . . . . . . . . . . . . . 10

2.1.2 Physical Channel and Packet Definition . . . . . . . . . . . . 11

2.1.3 Physical Links . . . . . . . . . . . . . . . . . 12 
2.1.4 Modulation Scheme . . . . . . . . . . . . . . . . 13

2.1.5 Transmission Model . . . . . . . . . . . . . . 16

2.1 .6 Error Correction . . . . . . . . . . . . . . . 20

2.2 Bluetooth Receivers Literature Review . . . . . . . . . . . . . . 21

2.2 .1 Optimum Receiver . . . . . . . . . . . . . . . . 22

2.2 .2 Suboptimum Receivers . . . . . . . . . . . . . . 27

2.3 MLSD Lower Bound . . . . . . . . . . . . . . . . . . . . . . 31

3 Noncoherent Decision Feedback Equalizer Receiver Structure 33

3.1 Laurent's Representation of CPM Signals . . . . . . . . . . . . . . . . . 34

3.1.1 Laurent's Decomposition and Its Application to Bluetooth . . . 35

3.1.2 Optimum Coherent Receiver Based on Laurent's Decomposition 39

3.1.3 Main Pulse . . . . . . . . . . . . . . . . . . . . . 41

3.2 Filter Design . . . . . . . . . . . . . . . . . 43

3.3 Discrete-time Transmission Model . . . . . . . . . . . . . . 47

3.4 Reduced-State Sequence Estimation . . . . . . . . . . . . . . . . . . . . 48

3.5 Decision-Feedback Equalizer Structure . . . . . . . . . . . . . 50

3.5.1 Minimum Phase Channel . . . . . . . . . . . . . . 51

3.5 .2 DFE Decision Rule . . . . . . . . . . . . . . . 53

3.6 Noncoherent Detection . . . . . . . . . . . . . . . . . . . . 54

3.6.1 NDFE Decision Rule . . . . . . . . . . . . . . . 57

3.6.2 Frequency Offset Estimation . . . . . . . . . . . . . . 58

3.6 .3 Adaptive NDFE . . . . . . . . . . . . . . . . . 58

3.7 Summary of Proposed Receiver Structure . . . . . . . . . . . . . . 60

4 Performance Results and Discussion $\quad 62$

4.1 Coherent Detection . . . . . . . . . . . . . . . . 63

4.1 .1 Filter Selection ................. 63

4.1.2 State Reduction . . . . . . . . . . . . . . . 65 
4.2 Noncoherent Detection . . . . . . . . . . . . . . . . 66

4.2.1 Performance with Constant Channel Phase . . . . . . . . . . . . 67

4.2.2 Performance Using the Modified Phase Reference Under Constant and Varying Channel Conditions . . . . . . . . 75

4.2 .3 Adaptive NDFE . . . . . . . . . . . . . . . . . . 80

4.2.4 Performance in the Presence of Interference . . . . . . . . . . . 83

5 Conclusions $\quad 86$

Bibliography $\quad 89$ 


\section{List of Tables}

2.1 Interference performance $[51] \ldots \ldots \ldots \ldots \ldots$

2.2 The values of relatively-prime integers $m$ and $p$ corresponding to different modulation indices $h$ within the range specified by the Bluetooth

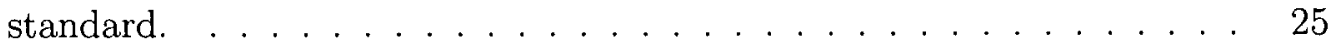

3.1 Duration of Laurent components $C_{P}(t) \ldots \ldots \ldots \ldots \ldots$ 


\section{List of Figures}

1.1 The required $10 \log _{10}\left(E_{b} / N_{0}\right)$ for $\mathrm{BER}=10^{-3}$ as a function of the modulation index $h \ldots \ldots \ldots \ldots \ldots$

2.1 General basic rate packet format [51]. . . . . . . . . . . . . . . 11

2.2 General enhanced data rate packet format [51] . . . . . . . . . . . 12

2.3 Block diagram of a CPM modulator. . . . . . . . . . . . . . 14

2.4 Frequency pulse shape $g(t)$ and phase pulse $q(t)$ for Gaussian frequency shift keying with time-bandwidth product $B T=0.5 \ldots \ldots . . . . .15$

2.5 Block diagram representing the ECB system model. . . . . . . . . . . . 19

2.6 Illustration of Repetition code. . . . . . . . . . . . . . . 21

2.7 Phase state trellis structure for Bluetooth with $h=1 / 3 . \ldots$. . . . . 26

2.8 LDI detector block diagram. . . . . . . . . . . . . . . . . . . . . . . . 29

2.9 Block diagram of the GFSK modulator using the Rimoldi/Huber\&Liu decomposition approach. ................... 31

3.1 Laurent pulses $C_{0}(t)$ and $C_{1}(t)$, and the main pulse $P(t)$ for Bluetooth GFSK signals with $L=2$ and $h=1 / 3 \ldots \ldots . \ldots . \ldots . . \ldots 39$

3.2 Block diagram of optimum coherent receiver based on Laurent's decomposition. . . . . . . . . . . . . . . . . . 40

3.3 The imaginary and real components of an exact Bluetooth signal $s(t)$ and an approximate signal $\hat{s}(t)$ generated using only the main pulse $P(t) .42$ 
3.4 Impulse response of SRC, $h_{\mathrm{SRC}}(t)$, before Hamming window (a), and after Hamming window (b). . . . . . . . . . . . . . . .

3.5 Magnitude frequency response for WMF, $H_{\mathrm{WMF}}(f)$, assuming $h=0.28$ and $h=0.35$, and for SRC filter, $H_{\mathrm{SRC}}(f)$, with $\rho=0.3 \ldots . . . .46$

3.6 The CIR of the (a) ISI channel $h_{c}[k]$ compared to (b) its minimum phase equivalent $h_{0}[k] \ldots \ldots \ldots \ldots \ldots$. . . . . . . . . . . . . . 48

3.7 Block diagram of decision-feedback equalizer. . . . . . . . . . . . 51

3.8 The roots of $H_{c}(z)$ compared to the roots of its minimum phase equivalent $H_{0}(z) \ldots \ldots \ldots \ldots \ldots$

3.9 Structure of the proposed Bluetooth receiver. . . . . . . . . . . . 60

4.1. Performance comparison of the SRC and WMF as receiver filters. Coherent detection with $h=1 / 3$ is assumed. . . . . . . . . . . . 64

4.2 Evaluation of the state reduction in the proposed receiver. Coherent detection with $h=1 / 3$ is assumed.

4.3 Performance of NDFE with $\alpha=0.8$ with FBF and FFF fixed at the receiver, or varying according to the value of $h$ at the transmitter. . . . 66

4.4 Evaluation of the performance of the proposed receiver with state reduction in case of noncoherent detection. . . . . . . . . . . . . 67

4.5 Performance of the NDFE using the $N$-metric compared to MLSD, LDI, and coherent DFE, and $h=1 / 3$ for all cases. . . . . . . . . . . . . 69

4.6 Performance of the NDFE using the $\alpha$-metric compared to MLSD, LDI, and coherent DFE, and $h=1 / 3$ for all cases. . . . . . . . . . .

4.7. Performance of the NDFE using the $N$-metric $h=1 / 3$ in the presence of phase jitter. . . . . . . . . . . . . . . . . . 70

4.8 Performance of NDFE using the $\alpha$-metric $h=1 / 3$ in the presence of phase jitter. . . . . . . . . . . . . . . 
4.9 Performance of the NDFE with $\alpha=0.8$ for different values of the modulation index compared to the MLSD bound, LDI, and MLM-LDI. . . . 72

4.10 Performance of the NDFE for varying $h$ with $\alpha=0.4,0.6,0.8$, and 0.9 . 73

4.11 Performance of NDFE for varying $h$ with $\alpha=0.6$ and 0.8 compared to

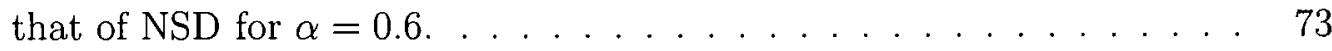

4.12 The required $10 \log _{10}\left(E_{b} / N_{0}\right)$ for $\mathrm{BER}=10^{-3}$ for NDFE with $\alpha=0.8$ with varying modulation index. . . . . . . . . . . . . 75

$4.1310 \log _{10}\left(E_{b} / N_{0}\right)$ required for $\mathrm{BER}=10^{-3}$ for different combinations of $\alpha$ and $\beta$ as a function of the modulation index with constant channel phase and $h$ known. . . . . . . . . . . . . . . 76

4.14 The required $10 \log _{10}\left(E_{b} / N_{0}\right)$ for $\mathrm{BER}=10^{-3}$ for $\mathrm{NDFE}$ with $\alpha=$ $0.6, \beta=0.9$ for varying $h$.

4.15 The required $10 \log _{10}\left(E_{b} / N_{0}\right)$ for BER $=10^{-3}$ for NDFE with $\alpha=$ $0.8, \beta=0.9$ for varying $h \ldots \ldots \ldots . \ldots \ldots$

4.16 The required $10 \log _{10}\left(E_{b} / N_{0}\right)$ for $\mathrm{BER}=10^{-3}$ in the presence of frequency offset and phase jitter.

4.17 The required $10 \log _{10}\left(E_{b} / N_{0}\right)$ for $\mathrm{BER}=10^{-3}$ in the presence of frequency offset and phase jitter.

$4.1810 \log _{10}\left(E_{b} / N_{0}\right)$ required for BER $=10^{-3}$ for ANDFE with phase reference (3.46). The channel phase is constant and the notation $\operatorname{ANDFE}\left(N_{e}, \mathcal{H}\right)$ applies. .

$4.1910 \log _{10}\left(E_{b} / N_{0}\right)$ required for $\mathrm{BER}=10^{-3}$ for $\mathrm{ANDFE}$ with various combinations of $\alpha$ and $\beta$ with phase reference (3.53). The channel phase is constant and the notation $\operatorname{ANDFE}\left(N_{e}, \mathcal{H}\right)$ applies. . . . . . . . .

$4.2010 \log _{10}\left(E_{b} / N_{0}\right)$ required for BER $=10^{-3}$ for ANDFE and ANSD with the favorable pair ( $\alpha=0.6, \beta=0.9$ ) and with constant channel phase. 
4.21 Performance of NDFE in the presence of interference for the WMF and the SRC filter. The channel phase is constant and the notation $\mathrm{IF}\left(\mathrm{SIR}, \Delta f_{c, i}\right)$ applies. . . . . . . . . . . . . . . . . 84

$4.2210 \log _{10}\left(E_{b} / N_{0}\right)$ required for BER $=10^{-3}$ for NDFE and ANDFE in the presence of interference. The channel phase is constant and the notation $\operatorname{IF}\left(\mathrm{SIR}, \Delta f_{c, i}\right)$ applies. . . . . . . . . . . . . . . . . 84 


\section{Glossary}

\section{List of Abbreviations}

(In alphabetical order)

ACL Asynchronous connectionless link

ANDFE Adaptive noncoherent decision-feedback equalizer

AMP Amplitude modulated pulses

ARQ Automatic retransmission query

AWGN Additive white Gaussian noise

BER Bit error rate

CPM Continuous phase modulation

CRC Cyclic redundancy check

DFE Decision-feedback equalizer

DM Medium data rate

DV Data voice

ECB Equivalent complex baseband

EDR Enhanced data rate

EV Extended voice

eSCO extended synchronous connection-oriented

FBF Feedback filter

FCC Federal Communications Commission

FEC Forward error correction 
FHS $\quad$ Frequency hop synchronization

FIR Finite impulse response

FFF Feedforward filter

GFSK Gaussian frequency shift keying

HEC Header error check

HV High-quality voice

ISI Intersymbol interference

ISM Industrial, scientific, medical

LDI Limiter-discriminator integrator

$\operatorname{MLS}(\mathrm{D} / \mathrm{E}) \quad$ Maximum-likelihood sequence (detector/estimator)

NDFE Noncoherent decision-feedback equalizer

NRSSE Noncoherent reduced-state sequence estimation

NSD Noncoherent sequence detector

PSK Phase shift keying

PSP Per-survivor processing

RSSE Reduced-state sequence estimation

SCO Synchronous connection-oriented

SIG Special interest group

SIR Signal-to-interference power ratio

SD Sequence detector

SRC Square-root raised cosine

UWB Ultra-wide band

VA Viterbi algorithm

WLAN Wireless local area network

WMF Whitened matched filter 


\section{Operators and Notation}

$t$ continuous time

[k] Discrete time

a Input binary data vector of $a[k] \in\{ \pm 1\}$

$b[k] \quad$ Zeroth complex symbols

$b_{P}[k] \quad$ Complex symbols

$B \quad 3-\mathrm{dB}$ bandwidth

$C_{P}(t) \quad$ Laurent pulses

$\exp \quad$ Exponential function

$E_{b} \quad$ Received energy per bit

$E_{s} \quad$ Signal energy per modulation interval

$f[k] \quad(\mathrm{N}) \mathrm{DFE}$ feedforward filter

$f_{c} \quad$ Carrier frequency

$f_{d} \quad$ Frequency deviation with respect to the carrier frequency

$g(t) \quad$ Frequency shaping pulse

$h \quad$ Modulation index

$h(t) \quad$ Gaussian low-pass filter

$h_{0}[k] \quad$ Minimum phase equivalent of $h_{c}[k]$, the (N)DFE feedback filter

$h_{c}[k] \quad$ Discrete-time channel impulse response (ISI channel)

$h_{S R C}(t) \quad$ Impulse response of the SRC filter

$h_{W M F}(t)$ Impulse response of the WMF

$H_{S R C}(f)$ Frequency response of the SRC filter

$H_{W M F}(f)$ Frequency response of the WMF

$\mathcal{H} \quad$ Number of modulation index hypotheses used in ANDFE

$i(t) \quad$ Equivalent complex baseband representation of interfering signal

$s_{R F}(t) \quad$ Passband representation of transmitted signal

$I_{0}($.$) \quad Modified Bessel function of first kind and zero order$ 
$K \quad$ Rician factor

$\log \quad$ Base-10 logarithm

ln Natural logarithm

$L \quad$ CPM memory

$m \quad$ the relatively prime numerator integer such that $h=m / p$

$n(t) \quad$ Additive white Gaussian noise

$N_{0} \quad$ One-sided noise power spectral density of the passband noise process

$N_{e} \quad$ Length of estimation period used in ANDFE

$p \quad$ the relatively prime denominator integer such that $h=m / p$

$P(t) \quad$ Laurent main pulse

$q(t) \quad$ Normalized phase pulse

$q_{\text {ref }} \quad$ Phase reference used in noncoherent detection

$Q(t) \quad$ Gaussian $Q$-function

$r(t) \quad$ Equivalent complex baseband representation of received signal

$r_{\mathrm{DFE}}[k] \quad$ output of feedforward filter

$\operatorname{rect}(t / T)$ rectangular pulse

$s(t) \quad$ Equivalent complex baseband representation of transmitted signal

$S_{n}(t) \quad$ Special sin functions used in Laurent's derivation process

$T \quad$ Symbol period

$\alpha \quad$ Phase estimation forgetting factor

$\beta \quad$ Frequency offset estimation forgetting factor

$\Delta f \quad$ Frequency offset between the transmitter and receiver oscillator

$\Delta f_{c, i} \quad$ frequency offset of the desired signal compared to the interfering signal

$\theta_{0} \quad$ Constant phase

$\rho \quad$ Roll-off factor of the SRC filter

$\phi_{0} \quad$ Constant phase rotation as a result of fading

$\phi(t) \quad$ Time-varying phase

$\varphi(t) \quad$ Phase function $\varphi(t)=2 \pi h q(t)$ 
$\psi(t, \boldsymbol{a}) \quad$ Information-carrying phase

$\Psi(t) \quad$ Generalized phase pulse function 


\section{Acknowledgements}

I would like to express my gratitude to my research supervisors, Dr. Lutz Lampe and Dr. Robert Schober, for their guidance and encouragement, and their valuable contribution to this research work. Their productive supervision throughout the past two years not only helped me broaden my scope of knowledge, but also increased my appreciation and dedication to this challenging research project, which has proven to be highly rewarding.

I also gratefully acknowledge the financial support in the forms of RA from Dr. Lutz Lampe and Dr. Robert Schober through NSERC grants STPGP 257684 and RGPIN 283152-04, and TA from the Electrical and Computer Engineering Department.

I would like to thank my colleagues in the communication theory group for sustaining a friendly work environment.

Finally, I would like to acknowledge the love and consistent support my family has provided me throughout my graduate studies in UBC. I would also like to thank my friends, especially Axel Davidian, for always being there, while being physically present at the other side of the globe. 


\section{Chapter 1}

\section{Introduction}

The pervasive use of mobile computing devices such as laptops and personal digital assistants (PDAs), and the evident success of cellular phones called for a wireless technology to connect these devices together. This technological vision became a reality with the introduction of the Bluetooth standard for wireless personal area networks (WPANs) which enables wireless communication among various electronic devices. Bluetooth has revolutionized the wireless world, for it provides low-power, low-cost, and short-range radio links with secure and reliable transmissions and global compatibility.

Bluetooth is an open standard, which enables manufacturers to take full advantage of the capabilities of the technology and build products according to its specifications, thus expanding the Bluetooth applications to diverse market areas. With seamless voice and data connections to virtually all mobile devices, the human imagination remains the only limit to application options. The Bluetooth technology specification, currently in its fourth version of the core specification, is developed by an industrybased association, the Bluetooth Special Interest Group (SIG), and will be outlined in detail in the next chapter.

The following section gives a brief history of the Bluetooth technology, its current 
status, and its future path. Section 1.2 states the motivation behind the present work and the challenges faced, and a summary of the various contributions is provided in Section 1.3. Finally, a brief description of the contents of the thesis concludes this chapter.

\subsection{The Evolution of the Bluetooth Technology}

In 1994, as the sales of cellular phones were increasing, Ericsson, one of leading telecommunications manufacturers, was investigating ways to add value to its phones in the crowded market. Consequently, Ericsson mobile research lab in Lund, Sweden, initiated a study to investigate the feasibility of a low-power, low-cost wireless technology to connect mobile phones and their accessories without the need of cumbersome cables. The study rapidly progressed, and as researchers realized the developed technology can be used to exchange data among numerous wireless and wired devices, the study quickly turned into a large project, which was given an internal code name, "Bluetooth". Bluetooth is the English derivative of the Viking word Blatand, and was named after the $10^{\text {th }}$ century Danish Viking King Harald Blatand who united Denmark and Norway during a time of fighting. Bluetooth developers considered the name appropriate for the technology since they anticipated it will unite the telecommunications world, just as Blatand united his world [5].

To allow Bluetooth to be an accepted industry standard, five major companies from three diverse business areas formed the Bluetooth Special Interest Group (SIG). The group was formally announced on May 20, 1998, and included two leading companies in mobile telephony, Ericsson and Nokia, two leading companies in laptop computing, IBM and Toshiba, and one leading company in digital signal processing, Intel. Today, the Bluetooth SIG has over 3,400 member companies all over the world [21]. In its mission statement, the Bluetooth SIG affirms that it "will support a collaborative 
environment and drive programs to develop and advance Bluetooth wireless technology in order to exceed personal connectivity expectations and meet the needs of a changing world". Versions 1.0, 1.0b, and 1.1 of the Bluetooth specifications were released in 1999, 2000, 2001, respectively. Bluetooth received additional support when the IEEE Standards Association approved the IEEE Std. 802.15.1, derived from the Bluetooth Specification, in March, 2002. The standard was published three months later, and introduced minor changes to the physical and medium access control (MAC) layers. Version 1.2 of the Bluetooth specification was formally ratified on November 2003, while the latest Bluetooth Core Specification Version 2.0+ Enhanced Data Rate (EDR) was released a year later, and provided increased data rates and lower power consumption.

The market for Bluetooth devices has been rapidly growing, and, as a result, the technology has met the significant milestone of five million Bluetooth units shipped per week. The number of Bluetooth-enabled devices doubled from 2003 to 2004, and is expected to reach 500 million units by the end of 2005. Moreover, a recent study conducted in Japan, United states, and United Kingdom has shown an increased customer awareness of Bluetooth.

Another emerging technology, ultra-wide band (UWB) appears to have great potential for the wireless applications which currently use Bluetooth. UWB transmits with very low power at extremely large bandwidths, but there are still some challenges before this technology can be realized. To meet the future market demands, and take advantage of the high data rates that UWB offers, the Bluetooth SIG announced in May, 2005 the collaboration of the Bluetooth and UWB developers. The goal is to employ UWB in the next-generation Bluetooth products, while maintaining backward compatibility with the existing products [1]. 


\subsection{Challenges and Motivation}

The objective of the present work is to design a cost-effective, power-efficient, and structurally-simple Bluetooth receiver. The optimum Bluetooth receiver has very high structural and computational complexity, for it consists of a bank of matched filters followed by a coherent ${ }^{1}$ maximum likelihood sequence detector (MLSD) with a number of states varying according to the modulation index. Since the modulation index in Bluetooth systems is allowed to vary in a relatively wide range $(0.28 \leq h \leq 0.35)$, the corresponding number of states in the optimum receiver. broadly varies with a minimum of 12 (as will be detailed in the next chapter), making it unfeasible. The practical and simple alternative, namely, the limiter-discriminator integrator (LDI) receiver, currently used for Bluetooth devices is a simple, low-cost receiver. However, it is highly suboptimal, for it suffers a performance loss of more than $6 \mathrm{~dB}$ over the optimum receiver, as shown in Fig. 1.1. The $6 \mathrm{~dB}$ gap suggests that the LDI receiver consumes four times as much signal power as the optimum receiver to achieve the same bit error rate (BER).

In the present work, we investigate the feasibility of an alternative Bluetooth receiver design, motivated by the large possible power efficiency gains that may be achieved over the conventional LDI receiver. This requires overcoming the following challenges faced when designing a Bluetooth receiver.

The varying modulation index results in a varying trellis structure with a large number of states, which poses a serious challenge when considering trellis-based detection. In addition, the metrics required for the detection of the maximum likelihood symbol sequence are also dependent on $h$. However, the trellis-based receivers achieve significant performance improvements over the conventional LDI receiver. Hence, we investigate

\footnotetext{
${ }^{1}$ Coherent detection assumes perfect channel phase estimate at the receiver.
} 


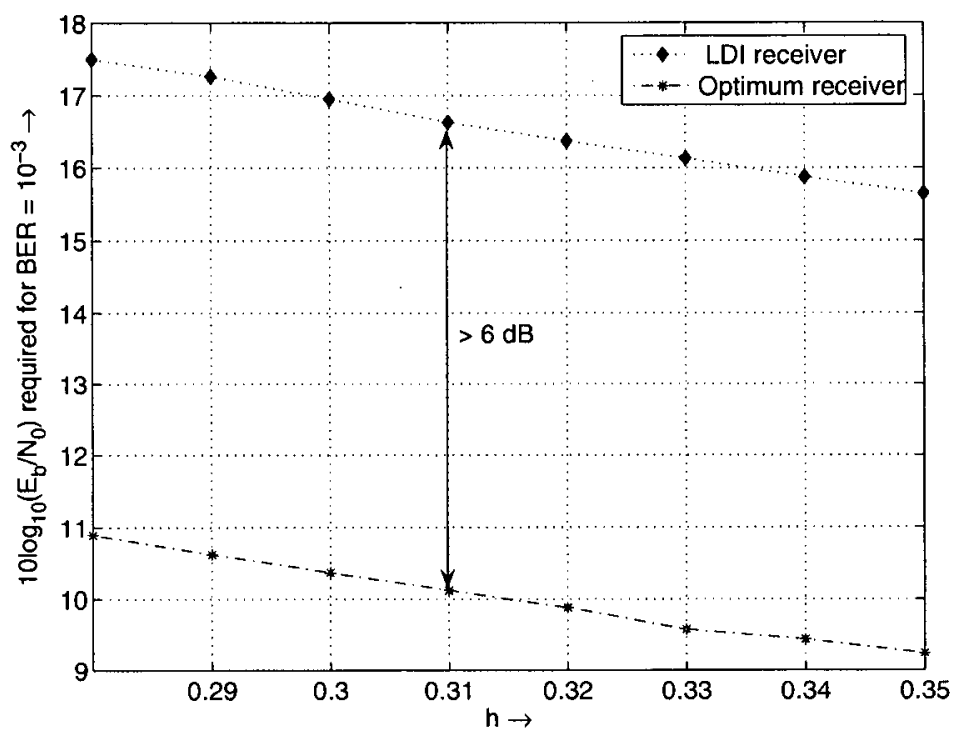

Figure 1.1: The required $10 \log _{10}\left(E_{b} / N_{0}\right)$ for $\mathrm{BER}=10^{-3}$ as a function of the modulation index $h$.

the design of a reduced-state sequence detector independent of the modulation index.

A carrier frequency deviation of up to $\pm 100 \mathrm{KHz}$ is allowed in Bluetooth systems [51], resulting in fast channel phase variations. Several coherent detectors which achieve significantly higher power efficiency compared to the LDI are available in the literature. However, perfect channel phase estimation is assumed at the receiver, which is impractical in Bluetooth systems. Therefore, we consider the suboptimum noncoherent detection schemes which perform implicit channel phase estimation.

Since Bluetooth operates in the license-free ISM band, it is vulnerable to interference from other Bluetooth and wireless local area network (WLAN) devices operating in the same frequency band. The design of the optimal receiver filter is dependent on the modulation indices, and, moreover, it is not guaranteed to accomplish strong outof-band interference suppression. The present work studies the design of a practical input receiver filter independent of $h$ and capable of accomplishing strong adjacent and co-channel interference suppression. 


\subsection{Contributions}

In the present work, we consider noncoherent sequence detection for Bluetooth signals based on Laurent's decomposition which models the GFSK signal as a superposition of amplitude modulation pulses, as will be described in Chapter 3 [32]. We then make the following contributions.

- As with Laurent's decomposition the actual nonlinear modulation scheme is transformed into a linear modulation over an intersymbol-interference (ISI) channel, we develop reduced-state trellis-based equalizers using the concepts of reducedstate sequence estimation (RSSE) [16] and per-survivor processing (PSP) [39], which will be presented in Chapter 3 . We also devise noncoherent versions of the RSSE and DFE receivers, which we refer to as noncoherent RSSE (NRSSE) and noncoherent DFE (NDFE).

- We propose the use of an off-the-shelf square-root raised cosine (SRC) filter as receiver input filter preceding symbol-rate sampling and RSSE, and we argue that this filter provides an almost sufficient statistic for the transmitted data.

- Using a seven-tap feedforward filter to obtain a minimum-phase channel impulse response, we show that decision-feedback equalization (DFE) achieves a performance close to the lower bound of maximum-likelihood sequence estimation (MLSE) ${ }^{2}$ [17]. This is a remarkable result, since complete state reduction incurred considerable performance degradation in case of an alternative noncoherent sequence detector (NSD) in the literature [31], [27]. We also note that a similar approach based on Laurent's decomposition required a 4-state trellis decoder to achieve a similar performance [43].

\footnotetext{
${ }^{2}$ In the context of the present work, "sequence estimation" and "sequence detection" are used synonymously.
} 
- We develop a simple frequency estimation method which provides high robustness to (extreme) frequency offsets and phase jitter.

- NRSSE and NDFE are extended to adapt the decision metric to the modulation index $h$ used at the transmitter side. This adaptive algorithm offers a simple solution to the problem of varying modulation index, and provides acceptable performance for all values of $h$.

- By means of simulation results for various transmission scenarios, we show that NDFE achieves almost the same performance as 2-state NSD. Due to its complexity advantage over NSD, the NDFE receiver is an attractive solution for a practical implementation.

\subsection{Thesis Outline}

This thesis is organized as follows.

Chapter 2 introduces the background information required for the implementation and evaluation of a Bluetooth receiver. Moreover, a literature review on the optimum and suboptimum Bluetooth receivers, as well as the MLSE lower bound are presented in order to provide solid grounds for comparison.

Chapter 3 describes in detail the structure of the proposed receiver. It starts by introducing Laurent's decomposition, which forms the basis of the present work. It points out the decomposition's major strengths and presents the optimum Bluetooth receiver based on the decomposition. After discussing the input receiver filter design, the corresponding discrete-time transmission model is introduced. The decision metric for coherent RSSE is then given, leading to the description of the structure of the extreme case of RSSE, the DFE. The minimum phase channel needed to achieve the maximum performance in reduced-state detectors is also discussed. The following 
section applies noncoherent detection to RSSE and DFE, through which phase and frequency estimation schemes are devised. The adaptive detector which estimates the modulation index at the receiver is then presented. We conclude this chapter by giving a brief summary of the proposed receiver structure.

Chapter 4 analyzes the performance of the proposed receiver in various scenarios and for various design parameters. After the evaluation of the proposed input receiver filter, the receiver performance with coherent and noncoherent detection is presented. Specifically, we study the effect of state reduction, and show the performance of the phase and frequency offset estimation methods under constant and varying channel conditions. We also show the effect of the varying modulation index, before the adaptive modulation index estimator is evaluated. The simulation of the receiver performance achieved in the presence of adjacent and co-channel interference concludes the chapter.

Chapter 5 concludes this dissertation by giving a brief summary of the key characteristics of the proposed receiver. 


\section{Chapter 2}

\section{Background}

This chapter provides the background information necessary for the design, analysis, and evaluation of the Bluetooth receiver presented thereafter. Bluetooth was designed to be a universal wireless technology, which operates at low power and can be implemented at low cost. Accordingly, the Bluetooth specifications [51] described in the first section of this chapter were carefully chosen so as to satisfy these requirements. Since the present work involves a receiver design, the second section provides a literature survey on the optimum and suboptimum Bluetooth receivers. These, in addition to the MLSE lower bound presented in Section 2.3 will serve as benchmarks when evaluating the proposed solution.

\subsection{The Bluetooth System}

This section elaborates on the Bluetooth specifications relevant for the present work. The radio specifications will be described first, followed by the physical channel and packet definition. The types of physical links available for data transmission as well as the modulation scheme used will then be presented. We conclude this section with an illustration of the channel model and an overview of the error correction schemes offered by Bluetooth. 


\subsubsection{Radio Front End}

Bluetooth operates in the $2.4 \mathrm{GHz}$ Industrial Scientific Medical (ISM) band, a frequency band that is globally available, license-free, and open to any radio system. The Bluetooth standard specifies a $1 \mathrm{MHz}$ bandwidth for each RF channel. However, there are variations in the range of frequencies available in the ISM band, and the Bluetooth specifications were defined to accommodate these differences. In most countries, where at least $80 \mathrm{MHz}$ of bandwidth is allocated to the ISM band, Bluetooth defines $79 \mathrm{RF}$ channels located at $(2402+k) \mathrm{MHz}, k=0,1, \ldots 78$. In other countries, such as France, Spain, and Japan, which allow the usage of only a reduced spectrum for the ISM band, 23 Bluetooth channels are defined, located at $(2454+k) \mathrm{MHz}, k=0,1, \ldots, 22$. Since Bluetooth devices do not have exclusive use of the frequency band, possible interference from other devices (garage door openers, baby monitors, microwave ovens, cordless phones, etc.) can be experienced. To minimize the effect of interference, a frequency-hopping spread spectrum approach is used. The radio hops through all the RF channels following a pseudo-random sequence [9], [13], [51]. Federal communications commission (FCC) regulations part 15.247 require that a device operates on a given channel for a maximum of 0.4 seconds within a 30 -second interval [34].

The Bluetooth core specification defines three transmit-power classes: $100 \mathrm{~mW}(20 \mathrm{dBm})$, $2.5 \mathrm{~mW}(4 \mathrm{dBm})$, and $1 \mathrm{~mW}(0 \mathrm{dBm})$, corresponding to Class 1,2 , and 3 devices, respectively. With Class 1 devices, the communication range may increase up to $100 \mathrm{~m}$. Power-save modes including Sniff, Hold, and Park (in decreasing order of power requirements) modes are used to reduce power consumption. Power control is used for devices with transmitter power higher than $4 \mathrm{dBm}$, and thus, necessary for Class 1 devices. For devices with lower transmitter power, power control is optional for optimizing the power consumption and the interference level [51]. 


\begin{tabular}{|l|l|l|}
\hline ACCESS CODE & HEADER & PAYLOAD \\
\hline
\end{tabular}

Figure 2.1: General basic rate packet format [51].

\subsubsection{Physical Channel and Packet Definition}

Bluetooth devices communicate with each other by forming piconets, which can accommodate up to eight devices. Several piconets can establish interconnections using bridge devices to form a larger network known as a scatternet. A piconet channel is divided into slots, each having a duration of $625 \mu \mathrm{sec}$. Users sharing the same channel are each assigned a time slot, and are time- and hop-synchronized to the channel to ensure reliable communication.

Information between Bluetooth devices is exchanged using packets. A packet may occupy one, three, or five time slots, and a frequency hop occurs only once the packet is complete. The hopping rate varies based on the number of slots occupied per packet, and reaches a maximum rate of 1600 hops/sec. The Bluetooth Standard defines sixteen different types of packets depending upon the application. In the Bluetooth specification Version $2.0+$ EDR, higher transmission rates (relative to the older Bluetooth specification versions) and corresponding packet formats were introduced. In addition to the basic rate of $1 \mathrm{Mbps}$, which uses the Gaussian frequency shift keying (GFSK) modulation scheme, an enhanced data rate with two new modulation modes was also defined. The primary modulation mode, $\pi / 4$-DQPSK (differential encoded quaternary phase shift keying), provides a data rate of $2 \mathrm{Mbps}$, while the secondary mode, 8-DPSK (differential encoded 8-ary phase shift keying), increases the data rate to up to $3 \mathrm{Mbps}$.

The general basic rate packet format is shown in Fig. 2.1. Each packet begins with an access code, unique for the channel. The access code consists of 4 Preamble bits, a 64-bit sync word and an optional 4-bit trailer appended to the sync word only if a header packet follows. The access code is used for synchronization, direct current (DC) 


\begin{tabular}{|c|c|c|c|c|c|}
\hline ACCESS CODE & HEADER & GUARD & SYNC & $\begin{array}{c}\text { ENHANCED DATA RATE } \\
\text { PAYLOAD }\end{array}$ & TRAILER \\
\hline
\end{tabular}

Figure 2.2: General enhanced data rate packet format [51].

offset compensation, and identification. A 72-bit header may follow the access code. The header contains important control information such as packet type, flow control, and header error control (HEC), and is encoded with a $1 / 3$ forward error correction (FEC) code. The payload field has a length varying between 0 and 2745 bits [51].

The general enhanced date rate packet format is shown in Fig. 2.2. Each packet consists of an access code, a header, a guard period, a synchronization sequence, an enhanced data rate payload, and a trailer. As noted in the figure, the access code and the header use GFSK, the same modulation scheme used in basic rate packets. The guard time then allows for transition into the higher data rate modulation scheme. Since this dissertation only involves the basic data rate modulation scheme, only GFSK will be elaborated in the following section.

\subsubsection{Physical Links}

Bluetooth uses two types of links for voice and data transmission, the synchronous, connection-oriented (SCO) links and the asynchronous connectionless links (ACL). Bluetooth devices can use either link, depending on the type of packets being transmitted. Further, a device can alternate between the two types of links during transmission, as long as an ACL link is established before using an SCO link. ACL links support point-to-multipoint connections with a maximum data rate of $732.2 \mathrm{Kbps}$ in an asymmetrical mode or $433.9 \mathrm{Kbps}$ in a symmetrical mode and are primarily used for file and data transfers. SCO links support point-to-point connections with a data rate of $64 \mathrm{Kbps}$ and are typically reserved for voice traffic. The SCO link was further improved in Version 1.2 of the Bluetooth specification, by defining a new extended 
SCO (eSCO) link. New packet types were defined for this link as extended voice (EV) packets, which are the original high quality voice (HV) packets with cyclic redundancy check (CRC) enabled to achieve higher reliability. The eSCO may be used for transparent synchronous user data and audio transmissions, and data rates of $384 \mathrm{Kbps}$ and $564 \mathrm{Kbps}$ can be achieved. These data rates are the same as those enabled by the third generation (3G) Universal Mobile Telecommunications Service (UMTS) systems, making the eSCO the most suitable link for audio and video streaming applications over 3G networks [6], [22].

\subsubsection{Modulation Scheme}

Gaussian frequency shift keying (GFSK), a special case of continuous phase modulation (CPM), is the modulation scheme used in the basic data rate mode of Bluetooth (cf. Section 2.1.2). CPM has been widely used in communications due to its power and bandwidth efficiency as well as its constant envelope. Constant envelope modulation schemes are known to allow for efficient power amplification. Further, CPM provides better spectral utilization than frequency shift keying (FSK) by introducing a continuous phase to smooth variations between symbols. The block diagram of a CPM modulator is shown in Fig. 2.3, where the frequency modulator consists of a voltage controlled oscillator (VCO). The resulting CPM passband signal is represented by [2]

$$
s_{R F}(t)=\sqrt{\frac{2 E_{s}}{T}} \cos \left(2 \pi f_{c} t+\psi(t, \boldsymbol{a})+\theta_{0}\right),
$$

where $E_{s}$ denotes the signal energy per modulation interval $T, f_{c}$ is the carrier frequency, $\theta_{0}$ is a constant phase which may be ignored without loss of generality, and $\psi(t, \boldsymbol{a})$ is the information-carrying phase

$$
\psi(t, \boldsymbol{a})=2 \pi h \sum_{k=-\infty}^{\infty} a[k] q(t-k T)
$$

Here, $a[k] \in\{ \pm 1\}$ is the random binary data, $h$ is the modulation index, and $q(t)$, commonly referred to as the normalized phase pulse, is defined as $q(t)=\int_{-\infty}^{t} g(\tau) \mathrm{d} \tau$. 


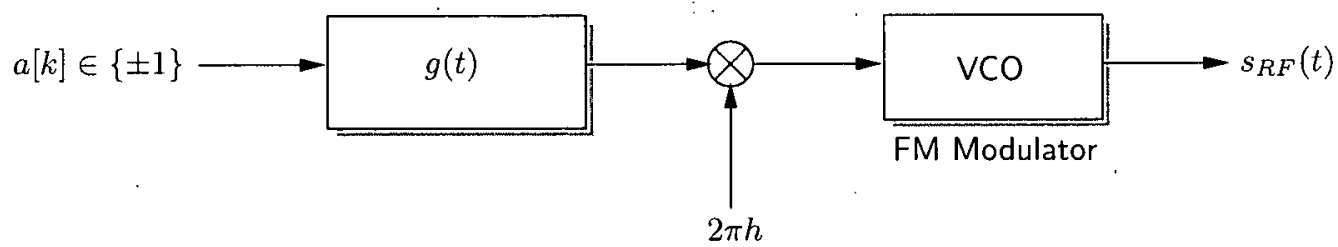

Figure 2.3: Block diagram of a CPM modulator.

The frequency shaping pulse $g(t)$ determines the smoothness of $\psi(t, \boldsymbol{a})$ and is assumed to be of finite duration $L T$, where $L$ is known as the CPM memory. A time-limited $g(t)$ leads to an appropriate representation of $q(t)$ as

$$
q(t)= \begin{cases}0 & t<0 \\ \int_{0}^{t} g(\tau) \mathrm{d} \tau & 0 \leq t \leq L T \\ 1 / 2 & t \geq L T\end{cases}
$$

When $L>1$, the frequency pulse exceeds the symbol interval $T$, leading to intersymbol interference (ISI). However, this approach, known as partial response signaling, is desirable in CPM since it introduces additional memory and allows for a narrower power spectrum with low spectral sidelobes. Varying the parameter $h$, and choosing different frequency shaping pulses, $g(t)$, results in various CPM schemes. Continuous phase frequency shift keying (CPFSK) signals, for example, use a rectangular pulse shape of duration $T$, but this causes sudden frequency transitions, resulting in a large bandwidth [42]. To achieve a more compact spectrum and smoother frequency transitions, the frequency shaping pulse in GFSK consists of a rectangular pulse pre-filtered by a Gaussian low-pass filter $h(t)$ as [40]

$$
g(t)=h(t) * \operatorname{rect}(t / T)
$$

where $*$ denotes the convolution and $h(t)$ is given by

$$
h(t)=\frac{1}{\sqrt{2 \pi} \sigma T} \exp \left(\frac{-t^{2}}{2 \sigma^{2} T^{2}}\right),
$$



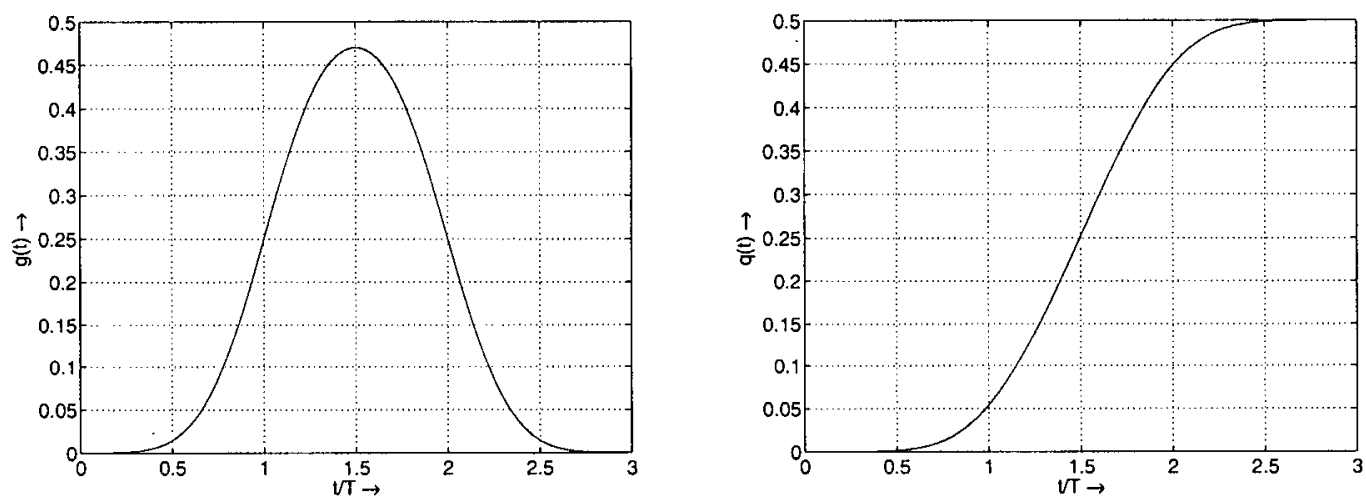

Figure 2.4: Frequency pulse shape $g(t)$ and phase pulse $q(t)$ for Gaussian frequency shift keying with time-bandwidth product $B T=0.5$.

with $\sigma=\sqrt{\ln (2)} /(2 \pi) B T$ and $B T$ is the 3-dB bandwidth-time product $(B$ : 3-dB bandwidth of the Gaussian filter). The result of the convolution of the Gaussian filter with the rectangular pulse that is defined as

$$
\operatorname{rect}\left(\frac{t}{T}\right)= \begin{cases}1 / T & \text { for }|t|<T / 2 \\ 0 & \text { otherwise }\end{cases}
$$

is the frequency pulse shape given by

$$
g(t)=\frac{1}{2 T}\left[Q\left(\frac{\sigma}{T}(t+T / 2)\right)-Q\left(\frac{\sigma}{T}(t-T / 2)\right)\right],
$$

where $Q(t)=(1 / \sqrt{2 \pi}) \int_{t}^{\infty} \mathrm{e}^{-\tau^{2} / 2} \mathrm{~d} \tau$ is often referred to as the Gaussian $Q$-function. The bandwidth of the GFSK spectrum and the resulting bit error rate at the receiver are both affected by two parameters: $B T$ and the modulation index $h=2 f_{d} T$, where $f_{d}$ is the frequency deviation (maximum frequency shift with respect to the carrier frequency). It is desirable to have a low $B T$ product and a small modulation index, for it results in a narrower spectrum. However, a low BT product produces higher ISI. In Bluetooth systems, $T=1 \mu \mathrm{sec}, B T=0.5$, and $f_{d}$ may vary between $140 \mathrm{KHz}$ and $175 \mathrm{KHz}$, resulting in $0.28 \leq h \leq 0.35$. The low modulation index values are due to 
the restrictions imposed by the FCC section 15.247 rule governing frequency hopping spread spectrum in ISM devices [51]. The GFSK frequency pulse shape $g(t)$ used in Bluetooth and the resulting phase pulse $q(t)$ are shown in Fig. 2.4.

\subsubsection{Transmission Model}

For short-range wireless systems, such as Bluetooth, communication often occurs in residential homes, office buildings, or commercial areas (factories, shopping centers, etc.). The signal propagation depends on the indoor environment and its topography, and varies with time due to the motion of people and equipment. In such settings, Doppler and delay spreads are minimal, resulting in a stationary or a slow-varying flatfading channel. The envelope of such channels is comparable to a Rician distribution. The probability density function of the Rician distribution is defined as [40]

$$
p(g)= \begin{cases}\frac{g}{\sigma^{2}} \mathrm{e}^{-\frac{g^{2}+A^{2}}{2 \sigma^{2}}} I_{0}\left(\frac{A g}{\sigma^{2}}\right) & \text { for }(g \geq 0) \\ 0 & \text { for }(g<0)\end{cases}
$$

where $g$ is the envelope of the channel gain, $A,(A \geq 0)$, is the amplitude of the dominant signal, $2 \sigma^{2}$ is the variance of the diffuse path, and $I_{0}(x)=1 / 2 \pi \int_{0}^{2 \pi} \exp (x \cos \phi) \mathrm{d} \phi$ is the modified Bessel function of first kind and zeroth order. The Rician distribution is described by a Rician factor $K=A^{2} / 2 \sigma^{2}$, which is the ratio of the power of the dominant path to the power of the diffuse path. In the special case of a weak dominant signal $(A \rightarrow 0)$, a Rayleigh distribution is obtained, while as $\sigma^{2} \rightarrow 0$, the result is an additive white Gaussian noise (AWGN) channel. The indoor propagation model in the 2.4 GHz unlicensed ISM band in which Bluetooth operates (cf. Section 2.1.1) was investigated in [29]. The root mean square (rms) of the delay spread was found to be below $70 \mathrm{nsec}$, with an average value of approximately $50 \mathrm{nsec}$. These values are very low compared to the symbol duration of $1 \mu \mathrm{sec}$ in Bluetooth. The channel propagation model was classified into two major categories. The first category includes channels with a line-of-sight path, which may be approximated by a Rician distribution with 
$K=5(\simeq 7 \mathrm{~dB})$. The second category describes channels with an obstructed path, and was found to have the same distribution with $K=2(\simeq 3 \mathrm{~dB})$, which is very close to the Rayleigh distribution. In a slow-fading channel, and after being affected by noise, the equivalent complex baseband (ECB) representation of the received signal is given by

$$
r(t)=g \mathrm{e}^{j \phi_{0}} s(t)+n(t)
$$

where $\phi_{0}$ is a phase rotation resulting from fading and is constant over time, $s(t)$ denotes the complex envelope of the transmitted signal that is written with a normalized unit amplitude as ${ }^{1}$

$$
s(t)=\exp (j \psi(t, \boldsymbol{a}))
$$

and $n(t)$ is additive white Gaussian noise (AWGN) with two-sided power spectral density $N_{0} / 2$. We note that the passband signal $s_{R F}(t)(2.1)$ can be written in terms of $s(t)$ (prior to amplitude normalization) as $s_{R F}(t)=\Re\left\{s(t) \mathrm{e}^{j 2 \pi f_{c} t}\right\}$, where $\Re($.$) represents$ the real part of the signal.

Oscillators are used to frequency modulate the Bluetooth information-carrying signal (cf. Section 2.1.4). The frequency stability of an oscillator is defined as the measure of the degree to which an oscillator maintains the same value of frequency over a given time. Oscillator instability results in a phenomenon known as phase noise or timing jitter, which introduces an additional time-varying phase rotation to the signal, $\Delta \phi(t, \tau)$, where $\Delta \phi(t, \tau)$ is a zero-mean Gaussian variable with a variance $\sigma_{\Delta}^{2}(\tau)$ depending on the employed oscillator and linearly increasing with time. Further, frequency fluctuations of a signal occur due to the frequency offset $(\Delta f)$ between the transmitter and receiver oscillator, contributing to a greater phase variation [14], [33]. The resulting time-varying phase is represented as a function of the aforementioned parameters as

$$
\phi(t+\tau)=\phi(t)+2 \pi \Delta f \tau+\Delta \phi(t, \tau) .
$$

\footnotetext{
${ }^{1}$ The constant phase term $\theta_{0}$ has been ignored.
} 
Table 2.1: Interference performance [51].

\begin{tabular}{|l|c|}
\hline Frequency of Interference $\left(\Delta f_{c, i}\right)$ & $\mathrm{SIR}$ \\
\hline $0 \mathrm{MHz}$ (Co-channel interference) & $11 \mathrm{~dB}$ \\
$1 \mathrm{MHz}$ (adjacent interference) & $0 \mathrm{~dB}$ \\
$2 \mathrm{MHz}$ (adjacent interference) & $-30 \mathrm{~dB}$ \\
$\geq 3 \mathrm{MHz}$ (adjacent interference) & $-40 \mathrm{~dB}$ \\
\hline
\end{tabular}

Consequently, the received signal in the presence of frequency offset and phase jitter is expressed as

$$
r(t)=g \mathrm{e}^{j\left(\phi(t)+\phi_{0}\right)} s(t)+n(t) .
$$

Due to operation in the license-free ISM band and frequency reuse, Bluetooth can suffer from interference from other Bluetooth and WLAN devices. Co-channel interference occurs when two or more piconets occupy the same frequency at the same time, while adjacent channel interference occurs when two piconets operate at neighboring frequencies in the same band. The performance in the presence of interference is determined by measuring the signal-to-interference (SIR) power ratio. The Bluetooth standard requires that the bit error rate be less than or equal to $0.1 \%$ in the presence of an interfering signal at $f_{c}+\Delta f_{c, i}$ for all the SIR ratios shown in Table 2.1 [51]. $\Delta f_{c, i}$ represents the frequency offset of the interfering signal compared to the desired signal. After considering the interfering signal, the ECB representation of the received signal may be given by

$$
r(t)=g \mathrm{e}^{j\left(\phi(t)+\phi_{0}\right)} s(t)+n(t)+i(t)
$$

where $i(t)$ denotes the ECB representation of the interfering signal. In the present work, we investigate and implement a novel receiver design for Bluetooth systems, as mentioned in Section 1.3. Since the channel gain can be assumed constant for the duration of one packet, and since detection for different packets is performed indepen- 


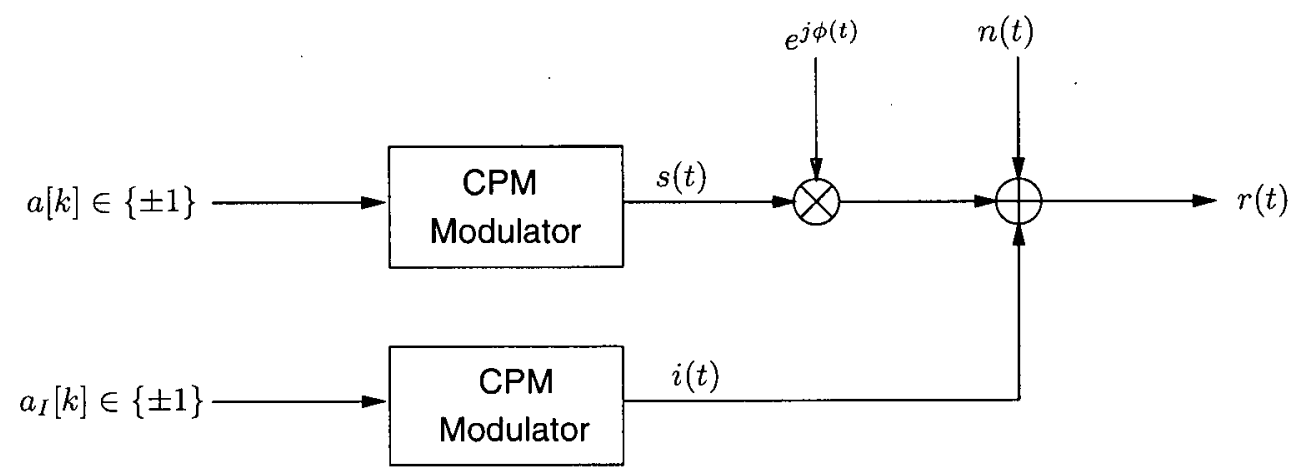

Figure 2.5: Block diagram representing the ECB system model.

dently, channel fading is irrelavant for receiver design. Consequently, in our design and after ignoring channel fading, the ECB representation of the received signal is

$$
r(t)=\mathrm{e}^{j \phi(t)} s(t)+n(t)+i(t) .
$$

A block diagram of the channel model is shown in Fig. 2.5, were $a_{I}[k]$ represents randomly-generated binary data symbols which are independent of $a[k]$. The CPM modulator was described in Section 2.1.4 and illustrated in Fig. 2.3.

A path loss model for the channel was defined in the IEEE standard 802.15.1 based on Bluetooth and approved by the IEEE in 2001 [26]. The path loss is represented as a function of the separation distance $d$ between the transmitter and receiver as follows

$$
L_{p}= \begin{cases}40.2+20 \log (d) & d<8 \mathrm{~m} \\ 58.5+33 \log (d / 8) & d>8 \mathrm{~m}\end{cases}
$$

where $L_{p}$ is the path loss in decibels $(\mathrm{dB})$. The corresponding received power $P_{R}$ is then obtained by subtracting the path loss from the transmitted power. The receiver sensitivity threshold in Bluetooth is $-70 \mathrm{dBm}$ with a nominal $0 \mathrm{dBm}$ transmit power. 


\subsubsection{Error Correction}

Bluetooth packets are checked for errors or wrong delivery using the channel access code, the HEC in the header, and the CRC in the payload. In addition, three errorcorrection schemes are offered:

- $1 / 3$ rate $\mathrm{FEC}$

- $2 / 3$ rate $\mathrm{FEC}$

- Automatic retransmission query (ARQ)

The above schemes are used to transmit different parts of the packet. Bluetooth devices may switch between these schemes as the signal level improves or degrades, thus maintaining performance at different noise levels. The three schemes are described in the following.

The $1 / 3$ FEC code is used for the header in all packets, as well as the synchronous data fields in the high quality voice 1 (HV1) packets. A $(3,1)$ repetition code is employed, as shown in Fig. 2.6. A conventional FEC decoder uses hard-decision decoding where the most frequent bit value is taken as correct. This results in a single-error correcting code. It is clear that this code may cause unnecessary overhead and, thus, a decrease in efficiency if not required, for we transmit two extra bits with no additional information. For this reason, the Bluetooth developers kept the 1/3 FEC code optional. The presence or absence of the code depends on the packet type, defined in the 4-bit 'TYPE' field in the packet header. However, this code is always used to protect the header, for it contains important link information, and bit errors would have a greater impact on performance [51].

The $2 / 3$ FEC code is used in medium rate data (DM) packet, the data field of the data voice (DV) packets, the frequency hop synchronization (FHS) packet, the high data rate voice 2 (HV2) packet, and in the extended voice 4 (EV4) packet. It accepts 10 bits as input, and outputs 15 bits by using a $(15,10)$ shortened Hamming code with 


\begin{tabular}{|c|c|c|c|c|c|c|c|c|c|c|c|c|c|c|}
\hline$a_{1}$ & $a_{2}$ & $a_{3}$ & .... & $\longrightarrow$ & $a_{1}$ & $a_{1}$ & $a_{1}$ & $a_{2}$ & $a_{2}$ & $a_{2}$ & $a_{3}$ & $a_{3}$ & $a_{3}$ & $\cdots$ \\
\hline
\end{tabular}

Figure 2.6: Illustration of Repetition code.

the generator polynomial $g(D)=\left(D^{5} \oplus D^{4} \oplus D^{2} \oplus 1\right)$. If a packet contains less than 10 bits, zeros are appended to the codeword before applying the code. All single and double adjacent errors can be corrected through the 2/3 FEC code [51].

The ARQ scheme is used only on the payload of packets that have CRC. These include $\mathrm{DV}, \mathrm{DM}, \mathrm{DH}$, and EV packets. At the start of a new connection, the ARQN bit is initialized to zero (NACK). After transmission starts, the ARQN bit is set to one (ACK) upon the successful reception of a CRC packet. If the $\mathrm{HEC}$ or CRC of any packet fails, or if no access code is detected, the ARQN bit is again set to NACK. The data payload is retransmitted until a positive acknowledgement is received or a time-out is exceeded [51].

\subsection{Bluetooth Receivers Literature Review}

CPM is considered an attractive modulation choice for wireless systems. This is partly due to the continuous phase and memory inherent in the signal, providing protection from channel errors. However, these properties that make CPM attractive also present difficulties for receiver designs. The next sections discuss several approaches for Bluetooth receiver designs in literature. The optimum receiver is presented first to serve as a lower bound for the suboptimum receivers discussed in the following section. 


\subsubsection{Optimum Receiver}

The optimum receiver discussed here assumes coherent detection and an AWGN channel. It uses the maximum-likelihood sequence detector (MLSD), which determines the most likely sequence of received symbols given all possible combinations of transmitted symbols. The optimum algorithm for performing (MLSD), the Viterbi algorithm (VA) [18], is a trellis search algorithm originally proposed in 1967 for decoding convolutional codes [55]. The optimum CPM receiver exploits the general state trellis structure of CPM to perform MLSD. Due to the memory inherent in a CPM signal, the phase at one time instant is dependent on the data symbol at that time instant in addition to all the previous data symbols. This can be shown in the general CPM state trellis structure obtained by using the assumption that the frequency shaping pulse $g(t)$ is of finite duration $L T$ (cf. Section 2.1.4). The following trellis structure exist only when the modulation index $h$ is a rational number, and constitutes the basis for performing MLSD for CPM using the VA, as will be described later in the section.

Introducing the CPM memory, $L$, into Eq. (2.2), the information-carrying phase can be appropriately represented as

$$
\psi(t, \boldsymbol{a})=\pi h \sum_{k=-\infty}^{n-L} a[k]+2 \pi h \sum_{k=n-L+1}^{n} a[k] q(t-k T), \quad n T \leq t \leq(n+1) T .
$$

The first term of the right-hand side of Eq. (2.15) constitutes the accumulated phase up to time $(n-L) T$. It is referred to as the phase state and denoted hereafter by $\theta[n-L]$. Using the fact that $\theta[n-L]$ and $\theta[n-L] \bmod (2 \pi)$ are physically indistinguishable, and conveniently representing the modulation index $h$ in terms of two relatively prime integers $m$ and $p$ as $h=m / p$, the phase state becomes

$$
\begin{aligned}
\theta[n-L] & =\left(\pi \frac{m}{p}\left[\sum_{k=-\infty}^{n-L} a[k]\right]\right) \bmod (2 \pi) \\
& =\frac{\pi}{p}\left(\left[m \sum_{k=-\infty}^{n-L} a[k]\right] \bmod (2 p)\right) .
\end{aligned}
$$


The second term in Eq. (2.15) is a sum of the phase contribution due to the most recent symbol $a[n]$ and the state vector $(a[n-1], a[n-2], \ldots, a[n-L+1])$. Hence, the state of the CPM signal at time $t=n T$ can be uniquely defined by the combination of the phase state and the state vector as

$$
S_{n}=\{\theta[n-L], a[n-L+1], a[n-L], \ldots, a[n-1]\}
$$

Consequently, the number of states $N_{s}$ in the trellis is

$$
N_{s}= \begin{cases}p M^{L-1} & (\text { even } m) \\ 2 p M^{L-1} & (\text { odd } m)\end{cases}
$$

We have shown that a CPM signal can be represented by a trellis consisting of a finite number of states, which form what is referred to as a finite state machine (FSM) ${ }^{2}$. To perform detection through MLSD, the VA decides on the path metric which maximizes the $\log$-likelihood function $\log [\mathrm{p}(r(t) \mid s(t))]$, where $\mathrm{p}(r(t) \mid s(t))$ is the conditional probability density function (pdf) defined for AWGN channels by

$$
\mathrm{p}(r(t) \mid s(t)) \propto \exp \left(\frac{-1}{N_{0}} \int_{-\infty}^{+\infty}|r(t)-s(t)|^{2} \mathrm{~d} t\right) .
$$

Consequently, the MLSD decision rule is given by

$$
\begin{aligned}
\hat{\boldsymbol{a}} & =\underset{\tilde{\boldsymbol{a}}}{\operatorname{argmax}}\left\{-\int_{-\infty}^{+\infty}|r(t)-\tilde{s}(t)|^{2} \mathrm{~d} t\right\} \\
& =\underset{\tilde{\boldsymbol{a}}}{\operatorname{argmax}}\left\{-\int_{-\infty}^{+\infty}|r(t)|^{2} \mathrm{~d} t-\int_{-\infty}^{+\infty}|\tilde{s}(t)|^{2} \mathrm{~d} t+\int_{-\infty}^{+\infty} 2 \Re\left\{r(t) \cdot \tilde{s}^{*}(t)\right\}^{2} \mathrm{~d} t\right\}
\end{aligned}
$$

where $\hat{\boldsymbol{a}}$ denotes the estimated symbol sequence, $\tilde{\boldsymbol{a}}$ represents the trial symbol sequence, and $\tilde{s}(t)$ represents the hypothetical transmitted signal sequence associated to the hypothetical symbol sequence $\tilde{\boldsymbol{a}}$. We notice that in Eq. (2.21) the decision rule is

\footnotetext{
${ }^{2} \mathrm{~A}$ FSM is a model of computation consisting of a set of states, having an input alphabet and a transition function which maps the input symbols and current states to a next state.
} 
independent of $|r(t)|^{2}$, while $|\tilde{s}(t)|^{2}$ is a constant term since it is expressed in terms of an exponential (2.9). Hence, we can eliminate these terms and disregard the factor of 2 in the remaining term since it has no effect on the decision, resulting in the following MLSD decision rule

$$
\hat{\boldsymbol{a}}=\underset{\tilde{a}}{\operatorname{argmax}}\left\{\int_{-\infty}^{+\infty} \Re\left\{r(t) \cdot \tilde{s}^{*}(t)\right\} \mathrm{d} t\right\} .
$$

The above equation shows that the complexity of the MLSD increases exponentially with the length of the symbol sequence $\boldsymbol{a}$. This can be avoided by using the VA, which introduces a recursively calculated metric $\Lambda_{n}(\tilde{\boldsymbol{a}})$ as follọws

$$
\begin{aligned}
\Lambda_{n}(\tilde{\boldsymbol{a}}) & =\int_{-\infty}^{(n+1) T} \Re\left\{r(t) \cdot \tilde{s}^{*}(t)\right\} \mathrm{d} t \\
& =\Lambda_{n-1}(\tilde{\boldsymbol{a}})+\int_{n T}^{(n+1) T} \Re\left\{r(t) \cdot \tilde{s}^{*}(t)\right\} \mathrm{d} t
\end{aligned}
$$

where $\Lambda_{n-1}(\tilde{\boldsymbol{a}})$ is the accumulated metric of the surviving sequence up to time $t=n T$ and the second term on the right-hand side the equation, known as the branch metric $\lambda_{n}(\tilde{\boldsymbol{a}})$, is given by

$$
\lambda_{n}(\tilde{\boldsymbol{a}})=\int_{n T}^{(n+1) T} \Re\left\{r(t) \cdot \exp \left(-j\left[\theta[n-L]+2 \pi h \sum_{k=n-L+1}^{n} \tilde{a}[k] q(t-k T)\right]\right)\right\} \mathrm{d} t .
$$

We note that the branch metric is interpreted as filtering the received signal $r(t)$ through a bank of $2 M^{L}$ matched filters and sampling to obtain outputs which form sufficient statistics for detection. We notice that the complexity of the MLSD using the VA increases only linearly with the length of the symbol sequence. The VA can be best described with the illustration of its key steps: add, compare, and select (ACS). The decision rule as given by Eq. (2.22) shows that we have a maximum problem. Therefore, the ACS consists of the following steps [8]. 
Table 2.2: The values of relatively-prime integers $m$ and $p$ corresponding to different modulation indices $h$ within the range specified by the Bluetooth standard.

\begin{tabular}{|c|c|c|c|c|c|c|c|c|}
\hline$h$ & 0.28 & 0.29 & 0.30 & 0.31 & 0.32 & $0.3 \overline{3}$ & 0.34 & 0.35 \\
\hline$m$ & 7 & 29 & 3 & 31 & 8 & 1 & 17 & 7 \\
\hline$p$ & 25 & 100 & 10 & 100 & 25 & 3 & 50 & 20 \\
\hline
\end{tabular}

1. Add: At time $t=(n+1) T$, we examine the two branches which lead to a common state. For each of these branches, we add the calculated branch metric to the corresponding accumulated metric.

2. Compare: The result of the sums generated in step 1 are compared.

3. Select: The branch (and corresponding data symbol) associated with the maximum path metric is selected.

After performing the ACS step, the branch metric corresponding to the selected symbol, also known as the surviving metric, is retained. The VA repeats the ACS step till the end of the symbol sequence, with one out of $M$ branch metrics selected at each state. Hence, at each time instant, the VA computes all possible $p M^{L}$ (or $2 p M^{L}$ ) metrics $\Lambda_{n}(\tilde{\boldsymbol{a}})$ corresponding to all $M^{L}$ possible sequences and $p$ (or $2 p$ ) possible phase states. This results in $p M^{L-1}$ (or $2 p M^{L-1}$ ) surviving sequences at each time instant of the VA. The value of the final accumulated path metric corresponds to the maximum path metric value for all possible sequences of symbols. The symbol sequence estimate corresponding to this maximum path metric $\hat{\boldsymbol{a}}$ constitutes the output of the VA.

Considering the special case of Bluetooth with binary GFSK and $L=2$, the received vector is required to pass through $8\left(=2 M^{L}\right)$ filters prior to sampling. The state vector becomes $S_{n}=\{\theta[n-2], a[n-1]\}$. The variables needed to construct the trellis depend on the integers $m$ and $p$, which are given in Table 2.2 for various values of $h$ (in the range specified in the Bluetooth standard). Obviously, the minimum values for $m$ and 


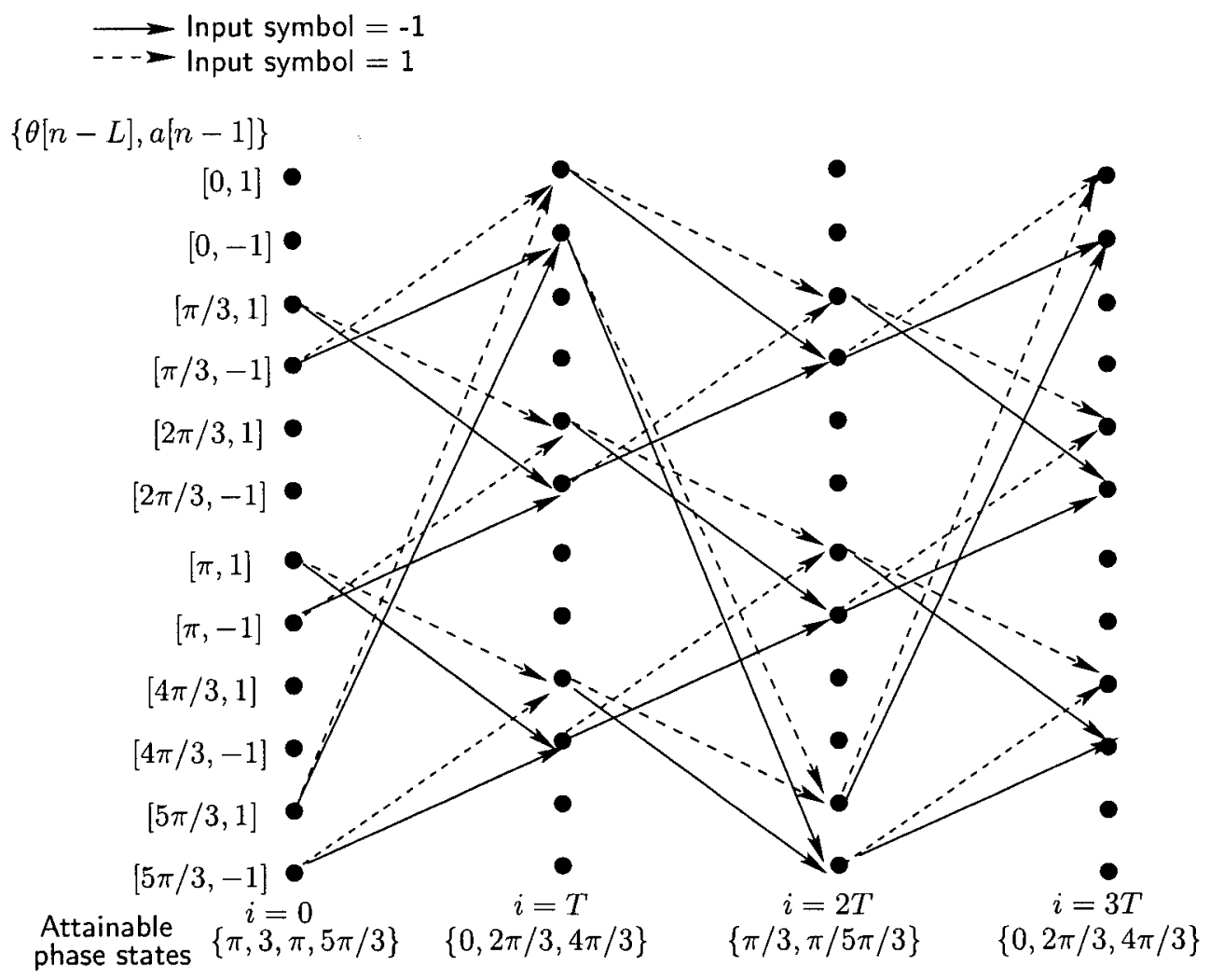

Figure 2.7: Phase state trellis structure for Bluetooth with $h=1 / 3$.

$p$ result in the least complex receiver.

To illustrate the complexity of the optimum receiver even in the simplest scenarios, we choose the case of $h=1 / 3$. For Bluetooth with $h=1 / 3$, there would be 12 states in the VA; each state is a unique combination of $a[n-1] \in\{ \pm 1\}$ and $\theta[n-2] \in$ $\{0, \pi / 3,2 \pi / 3, \pi, 4 \pi / 3,5 \pi / 3\}$. At each state, two trial symbols $\tilde{a}[n]=+1$ and $\tilde{a}[n]=-1$ are considered and the VA decides on the corresponding metric that maximizes (2.22). Hence, at each time instant of the VA, 24 metrics are calculated and 12 surviving metrics remain. The trellis structure for the optimum Bluetooth receiver for $h=1 / 3$ is shown in Fig. 2.7. For simplicity, the figure illustrates the VA paths at only three phase states at $i=0$. These phase states were carefully chosen, for they lead to three 
different phase states at the next symbol interval.

From Table 2.2, we notice that even a slight variation of $h$ can lead to a totally different trellis structure. Therefore, assuming a fixed nominal $h$ is not an option in this design. Moreover, the complexity of the optimum receiver, defined by the number of states $p M^{L-1}$ (or $2 p M^{L-1}$ ), increases exponentially as $M$ and $L$ increase, and tremendously varies with $h$. It also assumes perfect channel phase estimation, which is not valid in practice (cf. Section 2.1.5). We conclude that the optimum receiver performance may be considered as a benchmark, but it is definitely not a feasible solution for the Bluetooth receiver design.

\subsubsection{Suboptimum Receivers}

Since the optimum receiver has an extremely high complexity, Bluetooth devices often employ a simple discriminator receiver to recover the GFSK modulated data. This receiver uses a limiter-discriminator integrator, and, therefore, is referred to hereafter as the LDI receiver. It is adopted in Bluetooth due to its low computational and structural complexity, making it simple to implement at a low cost. However, the LDI suffers from a considerable performance loss $(>6 \mathrm{~dB})$ over the optimum receiver, as illustrated in Section 1.3.

Since the introduction of Bluetooth devices in the market, several receiver designs have been proposed in literature. The LDI-based designs in [47], [48], have only a slightly increased complexity; however, they offer only a slight increase in performance $(<1$ $\mathrm{dB}$ ). These include a receiver based on zero-crossing demodulation with a decorrelating matched filter [48], termed BT-ZXMF. Another design proposed by the same authors uses least squares-based post-integration filtering [47]. This approach is further extended in [49] by employing a max-log-maximum likelihood (MLM) symbol detection which involves a forward-backward algorithm on a 4-state trellis, and the resulting detector is termed MLM-LDI [49], [50]. Although a significant performance improvement 
$(\simeq 3.5 \mathrm{~dB})$ is observed, the design complexity generated by the postprocessing and the 4-state trellis is not acceptable for the low-cost Bluetooth devices.

As was observed in [49], sequence detection (SD) introduces tremendous performance gains since the memory of the CPM is properly taken into account. Consequently, many authors have considered trellis-based detection using the forward-backward algorithm [49] or the Viterbi algorithm [53], [43], [31]. A sequence detector based on Laurent's decomposition, termed Maximum aposteriori probability (MAP) receiver, was proposed in [43]. It consists of a filter matched to the first Laurent pulse, followed by a 4 -state trellis decoder. It performs better than all the previously-described receivers, reaching performance gains of approximately $6 \mathrm{~dB}$. However, it employs coherent detection, where a perfect channel phase estimate is assumed, and, therefore, it is impractical. In another SD design, [53], the trellis states in the Viterbi decoder were reduced to two by eliminating the effect of additional phase states. The main disadvantage of these trellis-based designs is that they assume a certain nominal value for the modulation index $h$. Since in Bluetooth $h$ is allowed to vary in a relatively wide range (cf. Section 2.1.4), assuming an incorrect $h$ at the receiver tremendously degrades the performance. Recently, a noncoherent sequence detector (NSD) which uses an adaptive algorithm that estimates the modulation index $h$ has been proposed [31]. It achieves a performance gain similar to the MAP receiver with a 2-state trellis. However, none of the sequence detectors proposed in literature offer performance gains (relative to the LDI) with complete state reduction. Specifically, a one-state trellis in the NSD receiver was shown to lead to a performance loss of more than $4 \mathrm{~dB}$ over the LDI receiver [27].

The two receivers relevant for comparison purposes are the LDI and NSD receivers, and, therefore, will be described in detail in the following. The LDI is considered a benchmark for all designed Bluetooth receivers since it is employed in state-of-the-art Bluetooth devices. The NSD receiver is relevant to the present work since, similar to 


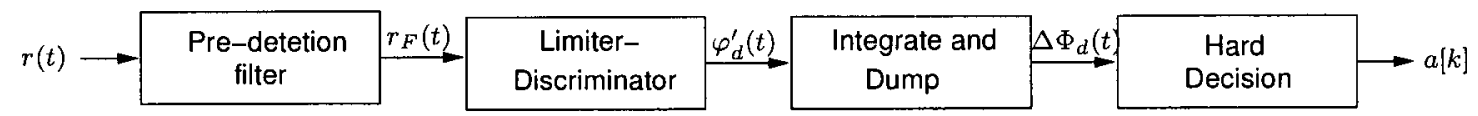

Figure 2.8: LDI detector block diagram.

the proposed receiver, it uses noncoherent sequence detection and a modulation index estimator.

\section{LDI detector}

The conventional limiter-discriminator integrator (LDI) detector is illustrated in Fig. 2.8. The received signal is first filtered by a pre-detection bandpass Gaussian filter with impulse frequency response [54]

$$
h_{r}(t)=\sqrt{\frac{2 \pi}{\ln 2}} B_{r} \mathrm{e}^{\left(\frac{2 \pi^{2}}{\ln 2}\right)\left(B_{r} t\right)^{2}}
$$

where $B_{r}$ is the $3-\mathrm{dB}$ bandwidth of the filter, with an optimum value of $1.1 \mathrm{MHz}$ in an AWGN channel [52]. The output of the Gaussian filter, $r_{F}(t)$, consists of a signal envelope, a distorted signal phase and a filtered noise term. The limiter-discriminator then outputs the derivative of the phase of $r_{F}(t), \varphi_{d}^{\prime}(t)$. The integrate and dump filter reintegrates the differentiated phase, producing a phase difference $\Delta \Phi_{d}(t)$, which represents the change over a symbol time of the signal phase plus the change in phase noise contributed by the AWGN. Hard decision is then performed on $\Delta \Phi_{d}(t)$, where a bit decision of ' 1 ' results if $\Delta \Phi_{d}(t)>0$, and ' 0 ' otherwise. The LDI detector was simulated in [27], and the obtained results will be used in this work for comparison purposes.

\section{NSD}

This receiver, described in [27], [31], is based on the Rimoldi/Huber\&Liu decomposition approach to CPM [41], [25]. It is shown in [41] that a CPM modulator can be 
decomposed into a trellis encoder and a signal mapper. The trellis encoder consists of shift registers with $L$ delay elements and a recursive phase integrator. A time-invariant trellis is achieved by introducing a slope function. Further, using a new reference frequency $f_{r}$ and modified unipolar information symbols $a_{m}[k]$, a modified phase state is obtained as [25]

$$
\Psi_{m}[n-L]=\left[m \sum_{k=-\infty}^{n-L} a_{m}[k]\right] \bmod (p) \in\{0,1, . ., p-1\} .
$$

From Eq. (2.26), we can deduce that the number of phase states is equal to $p$. Hence, the phase trellis for binary GFSK with $L=2$ would consists of $p M^{L-1}=2 p$ states and $p M^{L}=4 p$ possible trajectories in each time interval, resulting in $4 p$ time-limited, constant-envelope signal elements $\rho_{m\left(\boldsymbol{a}_{m}[k]\right)}(t)$, where $\boldsymbol{a}_{m}[k]=\left\{a_{m}[k], a_{m}[k-1], \Psi_{m}[n-\right.$ $L]\}$ is a unique address vector associated with each signal element. Applying this decomposition, the ECB transmit signal can be expressed as

$$
s(t)=\sum_{i=0}^{\infty} \rho_{m\left(\boldsymbol{a}_{m}[i]\right)}(t-i T) .
$$

The resulting block diagram of the GFSK modulator using the Rimoldi decomposition approach is illustrated in Fig. 2.9. The filter design for this receiver uses time-limited exponential functions as described in [25], [30]. In case of GFSK signals in Bluetooth, it was found in [27] that the use of only one filter; $h_{1}(t)$ yields excellent performance. The final filter consisted of a cascade of $h_{1}(t)$ and a Gaussian pre-filter.

In contrast to the previous designs, the concept of noncoherent detection [11] is adopted, eliminating the need for channel phase estimation. A decision metric which accounts for the time-varying phase and frequency offset was also developed. In addition, an adaptive sequence detector was designed to cope with the varying modulation index $h$. It was found that a 2-state Viterbi decoder provides significant performance improvement over the conventional LDI detector. NSD is shown to be robust against phase variations and frequency offset. The main disadvantage is the complexity required for 


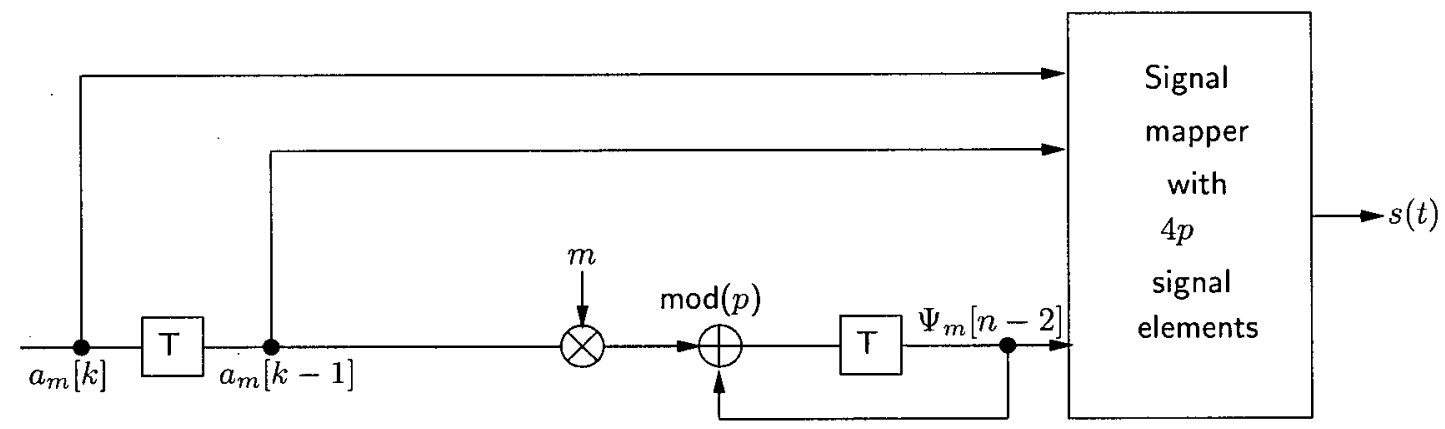

Figure 2.9: Block diagram of the GFSK modulator using the Rimoldi/Huber\&Liu decomposition approach.

a 2-state trellis search. With complete state reduction, the NSD performs poorly in terms of power efficiency [27]. The results presented in [27] will be used in the present work to illustrate the similarity in performance between NSD and the proposed receiver inspite of the proposed receiver's lower complexity.

\subsection{MLSD Lower Bound}

A lower bound for the performance of maximum likelihood sequence detectors for CPM signals was developed in [2]. This theoretical bound will be used to evaluate the proposed receiver in the results presented in Chapter 4. For high signal energy per bit-to-noise ratio (SNR), the bit error probability of coherent MLSD in an AWGN channel is given by [2]

$$
P_{e} \simeq Q\left(\sqrt{d_{\min }^{2} \frac{E_{b}}{N_{0}}}\right)
$$

where $d_{\min }$ is the minimum normalized Euclidean distance between two sequences of information symbols $a$, and $E_{b}$ is the received energy per bit. For CPM signals, the squared and normalized Euclidean distance measure is given by

$$
d^{2}=\log _{2}(M)\left\{\frac{1}{T} \int_{0}^{N T}[1-\cos \phi(t, \gamma)] \mathrm{d} t\right\}
$$


where $\phi(t, \gamma)=2 \pi h \sum_{i=0}^{\infty} \gamma[i] q(t-i T)$ and $\gamma[i]=a[i]-\tilde{a}[i]$ is the difference between the actual transmitted symbol $a[i]$ and the hypothetical symbol $\tilde{a}[i]$. Therefore, for binary symbols $\gamma[i] \in\{0, \pm 2\}$. We notice that this distance depends on the modulation index $h$.

It is clear from Eq. (2.29) that the Euclidean distance is a nondecreasing function of $N$, the number of symbol intervals. In [2, Chapter 3], an upper bound for the minimum $d, d_{\min }$, is obtained as a function of $h$. It represents the distance between the pair of sequences of infinite length that merges the earliest for any $h$. A 'merger' occurs once the phases of two sequences merge at a certain point in time, and coincide for all subsequent time intervals. If the merger between these two sequences occurs after $N_{m}$ symbol intervals, then the upper bound on the minimum squared Euclidean distance reads

$$
d_{\min }^{2}(h)=\log _{2}(M)\left\{\frac{1}{T} \int_{0}^{N_{m} T}\left[1-\cos \phi\left(t, \gamma_{\min }\right)\right] \mathrm{d} t\right\} .
$$

Using the above equation, a lower performance bound of MLSD is obtained by substituting (2.30) into (2.28) as

$$
P_{e} \geq Q\left(\sqrt{d_{\min }^{2}(h) \frac{E_{b}}{N_{0}}}\right)
$$

Since the proposed receiver employs sequence estimation, this bound is used for comparison purposes in Chapter 4 . 


\section{Chapter 3}

\section{Noncoherent Decision Feedback Equalizer Receiver Structure}

This chapter provides a description and analysis of the proposed receiver structure. The proposed receiver is designed to be structurally simple, yet power efficient and compliant with the Bluetooth specifications outlined in Chapter 2. As the nonlinear structure of the CPM signal presents various challenges for the receiver design, we adopt the linear representation of CPM, known as Laurent's decomposition. Section 3.1 explains the derivation process leading to Laurent's decomposition, which serves as the basis of receiver design. It further presents the corresponding optimum receiver and applies the linear representation to the Bluetooth GFSK signal. Section 3.2 discusses the proposed receiver input filter which, in addition to providing sufficient statistic for data detection, achieves strong interference suppression. In Section 3.3, the discretetime model is presented, resulting in an overall intersymbol interference channel.

To perform reliable detection, the proposed receiver must then employ an equalization scheme. Several equalization techniques have been proposed in literature, of which the MLSD and Maximum A Posteriori (MAP) are proven to be optimum. The MLSD is implemented using the Viterbi algorithm which determines the most likely symbol 
sequence as was detailed in Section 2.2.1. The MAP is implemented using the Bahl, Cocke, Jelinek, and Raviv (BCJR) algorithm [4] which determines the most probable symbol at a given time. However, due to the high computational complexity of these optimum methods, alternative low-complexity suboptimum schemes have been developed. In the present work, we employ a well-known suboptimum equalization scheme, reduced-state sequence estimation (RSSE), described in Section 3.4. Furthermore, we adopt a special case of RSSE, decision feedback equalization (DFE), of which an illustration is given in Section 3.5. To obtain a high performance with DFE, a minimum phase channel with maximum energy concentration in the first taps, is essential. Therefore, we employ an all-pass prefilter that transforms the ISI channel into a minimum phase channel, which serves as the feedback filter in the proposed DFE. For a practical implementation which accounts for the channel phase variations in Bluetooth signals, noncoherent detection, where the phase is implicitly estimated, is employed to the proposed RSSE and DFE. Section 3.6 describes the techniques used for the channel phase and frequency offset estimation. Moreover, since the modulation index varies in a relatively wide range in Bluetooth, an adaptive $h$-estimator is presented to tackle this problem. Finally, a brief summary of the proposed receiver structure is given in Section 3.7.

\subsection{Laurent's Representation of CPM Signals}

There are two alternative representations of CPM in literature. One representation given by Rimoldi in [41] decomposes the CPM into a trellis encoder and a signal mapper (cf. Section 2.2.2). This approach was adopted for the NSD receiver design described in [27]. As mentioned in Section. 2.2.2, the NSD receiver achieves high performance gains with a 2-state trellis, but performs worse than the conventional LDI in case of 
complete state reduction (1-state trellis).

Another decomposition approach, referred to as Laurent's decomposition, was proposed by Pierre Laurent in 1986 [32]. It represents the CPM signal either exactly or approximately as a linear superposition of a finite number of amplitude modulated pulses (AMP). Laurent's decomposition has been the basis of a considerable amount of research on CPM, including developing noncoherent sequence detection [11] and capacity bounds [56] for CPM. Moreover, using Laurent's representation, the challenges faced in the CPM receiver design due to the nonlinear structure of the signal are eliminated. As a result, it has been shown to be an effective tool for constructing reduced-complexity coherent [28], [10] and noncoherent [24], [11] CPM receivers. More specifically, a Bluetooth receiver based on Laurent's decomposition was proposed in [43], and achieved considerable performance gains over the conventional LDI. However, this receiver employed coherent detection, and the varying modulation index, channel phase variations, and frequency offsets were not addressed (cf. Section 2.2.2).

In the present work, we adopt Laurent's decomposition approach. The following sections describe the derivation which gives an exact representation of the CPM signal. Furthermore, we illustrate its application to the Bluetooth GFSK signal, discuss the corresponding optimum receiver, and present an approximate representation of the CPM signal.

\subsubsection{Laurent's Decomposition and Its Application to Blue- tooth}

Laurent developed an alternative representation for binary CPM signals, which expresses the CPM signal as a sum of $\left(2^{L-1}-1\right)$ pulse amplitude modulated (PAM) 
components ${ }^{1}$. The complete derivation process presented in [32] will be described in the following. For convenience, we define a new phase function $\varphi(t)=2 \pi h q(t)$, where $q(t)$ was given in (2.3), resulting in $\varphi(t)=\pi h$ for $t \geq L T$. The expression of $\psi(t, a)$ in the $n^{\text {th }}$ interval (2.15) can now be written as

$$
\psi(t, \boldsymbol{a})=\pi h \sum_{k=-\infty}^{n-L} a[k]+\sum_{k=n-L+1}^{n} a[k] \varphi(t-k T), \quad n T \leq t \leq(n+1) T .
$$

Recalling the ECB representation of the transmitted signal formulated in (2.9), and observing one signaling interval only, we may use Eq. (3.1) to express $s(t)$ in the $n^{\text {th }}$ interval as

$$
s(t)=\exp \left(j \pi h \sum_{k=-\infty}^{n-L} a[k]\right) . \prod_{k=n-L+1}^{n} \exp (j a[k] \varphi(t-k T)) .
$$

The next step in the derivation is referred to by Laurent as the 'most important step'. It involves the usage of Euler's formula in addition to exploiting the fact that $\cos (a[k] \varphi(t-$ $k T))=\cos (\varphi(t-k T))$ and $\sin (a[k] \varphi(t-k T))=a[k] \sin (\varphi(t-k T))$. After several mathematical manipulations, the complex exponential associated to the $m^{\text {th }}$ symbol can be defined as a sum of two terms

$$
\begin{aligned}
& \exp (j a[m] \varphi(t-m T))= \frac{\sin [\pi h-\varphi(t-m T)]}{\sin (\pi h)} \\
&+\exp (j \pi h a[m]) \frac{\sin [\varphi(t-m T)]}{\sin (\pi h)}, \\
& n-L+1 \leq m \leq n, n T \leq t \leq(n+1) T .
\end{aligned}
$$

We may notice that this formulation is not valid for integer values of $h$ since it would result in $\sin (\pi h)=0$. An alternative solution, proposed in [23], derives the AMP representation of a CPM signal with integer modulation index. We continue the derivation process by defining a generalized phase pulse function as

$$
\Psi(t)= \begin{cases}\varphi(t) & t<L T \\ \pi h-\varphi(t-L T) & t \geq L T\end{cases}
$$

\footnotetext{
${ }^{1}$ In this work, "Laurent pulses", and "Laurent components", and, in some cases, "AMP (or PAM) pulses (or components)", are used synonymously.
} 
In order to express Eq. (3.3) in terms of one function, we introduce the functions $S_{n}(t)$

$$
S_{n}(t)=\frac{\sin [\Psi(t+n T)]}{\sin (\pi h)}=S_{0}(t+n T) .
$$

Using these functions, Eq. (3.3) can be rewritten as

$$
\exp (j a[m] \varphi(t-m T))=S_{L-m}(t)+\exp (j \pi h a[m]) S_{-m}(t) .
$$

Introducing the above result in Eq. (3.2) leads to

$$
\begin{aligned}
s(t) & =\exp \left(j \pi h \sum_{k=-\infty}^{n-L} a[k]\right) \prod_{k=n-L+1}^{n}\left[S_{L-k}(t)+\exp (j \pi h a[k]) S_{-k}(t)\right] \\
& =\exp \left(j \pi h \sum_{k=-\infty}^{n-L} a[k]\right) \prod_{i=0}^{L-1}\left[S_{i+L-n}(t)+\exp (j \pi h a[n-i]) S_{i-n}(t)\right] .
\end{aligned}
$$

The product term on the right-hand side of Eq. (3.7) can be expanded into a sum of $2^{L}$ terms. However, a close examination of the result reveals that only $2^{L-1}$ of these terms are distinct functions of time. These form the AMP components that constitute Laurent's representation. To obtain a general form of these impulses, we define the radix-2 representation of the index $P$

$$
P=\sum_{i=1}^{L-1} 2^{i-1} \cdot \gamma_{P, i}, \quad 0 \leq P \leq 2^{L-1}-1
$$

where $\gamma_{P, i} \in\{0,1\}$. The AMP pulses are given by

$$
C_{P}(t)=S_{0}(t) \prod_{i=1}^{L-1} S_{i+L \cdot \gamma_{P, i}}(t) .
$$

The Laurent pulses $C_{P}(t)$ are nonzero in the interval $\left[0, \min _{i=1,2, \ldots L-1}\left(L\left(2-\gamma_{P, i}\right)-i\right)\right]$. Referring to Table 3.1, which shows the duration of the Laurent components, we notice that $C_{0}(t)$ has the longest duration. Laurent exploits this fact to represent the CPM signal in terms of only one impulse as will be shown in Section 3.1.3.

Finally, Laurent's representation of a CPM signal in terms of the AMP pulses reads

$$
s(t)=\sum_{k=-\infty}^{+\infty} \sum_{P=0}^{2^{L-1}-1} b_{P}[k] C_{P}(t-k T),
$$


Table 3.1: Duration of Laurent components $C_{P}(t)$.

\begin{tabular}{|l|c|}
\hline Laurent Component & Component duration \\
\hline$C_{0}(t)$ & $(L+1) T$ \\
$C_{1}(t)$ & $(L-1) T$ \\
$C_{2}(t), C_{3}(t)$ & $(L-2) T$ \\
$C_{4}(t), C_{5}(t), C_{6}(t), C_{7}(t)$ & $(L-3) T$ \\
$\ldots$ & \\
$C_{\left(2^{L-1}\right) / 2}, \ldots, C_{\left(2^{L-1}-1\right)}$ & $T$ \\
\hline
\end{tabular}

where the complex symbols $b_{P}[k]$, referred to in [35] as pseudo-symbols, are expressed in terms of the binary data symbols as

$$
b_{P}[k]=\exp \left(j \pi h\left(\sum_{l=-\infty}^{k} a[l]-\sum_{i=1}^{L-1} a[k-i] \cdot \gamma_{P, i}\right)\right)=\exp \left(j \pi h A_{P}[k]\right) .
$$

Eq. (3.10) is an exact representation of Eq. (2.9). However, as mentioned earlier Laurent's decomposition as derived above is restricted to binary CPM signals. The decomposition approach wạs further extended in [35] to M-ary signals.

Considering the special case of Bluetooth GFSK signals with $L=2$ and applying Laurent's decomposition, the resulting linear representation is given by

$$
s(t)=\sum_{k=-\infty}^{+\infty}\left(b_{0}[k] C_{0}(t-k T)+b_{1}[k] C_{1}(t-k T)\right)
$$

where $C_{0}(t)=S_{0}(t) S_{1}(t)$ and $C_{1}(t)=S_{0}(t) S_{3}(t)$ are illustrated in Fig. 3.1 for $h=1 / 3$. Since $L=2$ for Bluetooth, we notice that $C_{0}(t)$ has a duration of $3 T$, while $C_{1}(t)$ has a duration of $T$ and significantly less energy. The corresponding optimum receiver is described in the next section. 


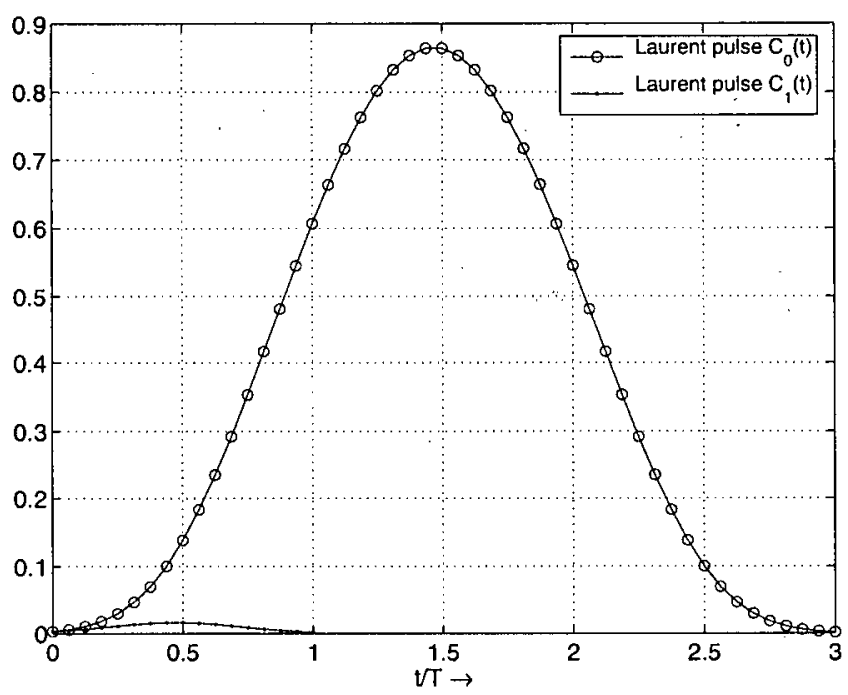

Figure 3.1: Laurent pulses $C_{0}(t)$ and $C_{1}(t)$, and the main pulse $P(t)$ for Bluetooth GFSK signals with $L=2$ and $h=1 / 3$.

\subsubsection{Optimum Coherent Receiver Based on Laurent's De- composition}

Using the linear representation of CPM given in Eq. (3.10), a new optimum coherent detector for binary CPM was developed in [28]. This receiver requires $2^{L-1}$ matched filters and a Viterbi sequence detector with $p 2^{L-1}$ states, as shown in Fig. 3.2. To obtain the VA state vector, we conveniently express the complex symbols (3.16) as follows. When $P=0, b_{0}[k]=b_{0}[k-1] \exp (j \pi h a[k])$, while for $P=1,2, \ldots 2^{L-1}-1$, the complex symbols are represented in terms of $b_{0}[k-L]$ as

$$
b_{P}[k-L]=b_{0}[k-L] \prod_{i \in I_{P}} \exp (j \pi h a[k-i])
$$

where $I_{P}$ is a nonempty subset of the set $\{0,1, \ldots, L-1\}$. Hence, the state of the CPM signal can be uniquely represented at every time instant by the vector $\left\{b_{0}[k-L], a[k-\right.$ $L+1], \ldots, a[k-2], a[k-1]\}$. Recalling that the modulation index $h$ may be represented as $h=m / p$, we deduce that $b_{0}[k-L]$ can take $p$ values. Taking into account the remaining $(L-1)$ binary symbols, it is evident that $p 2^{L-1}$ states are required for the 


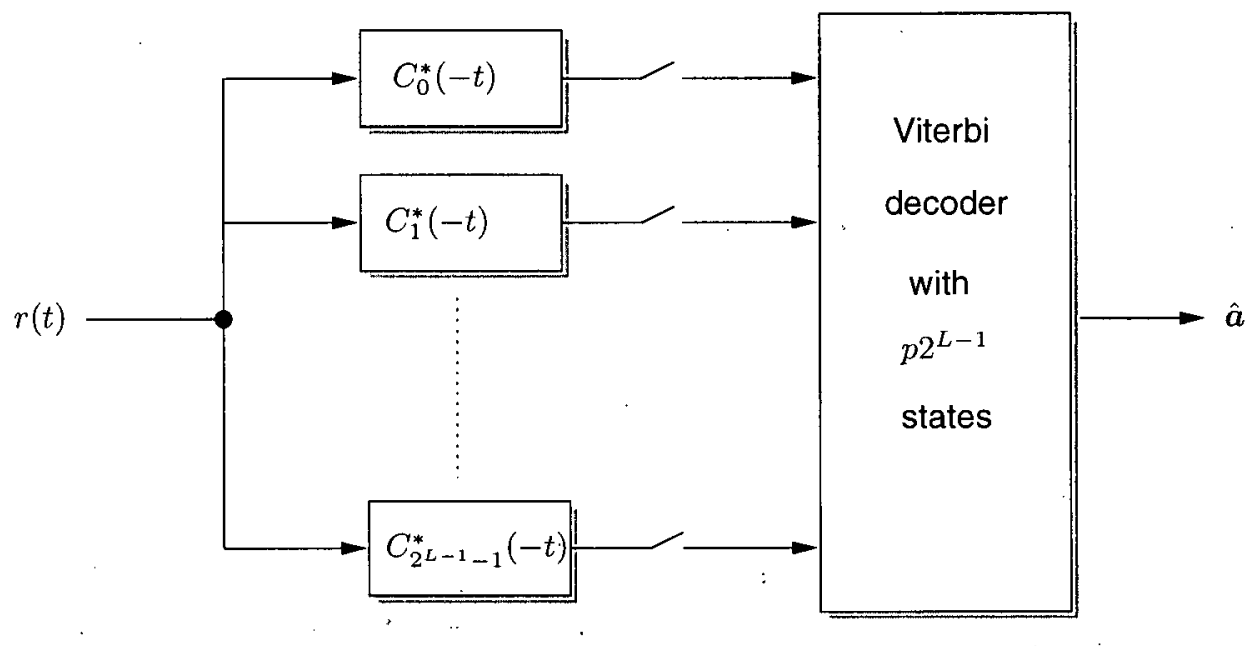

Figure 3.2: Block diagram of optimum coherent receiver based on Laurent's decomposition.

Viterbi algorithm.

For Bluetooth GFSK signals (3.12), the optimum receiver is implemented as follows. The received signal $r(t)$ is fed into a bank of 2 filters $C_{0}^{*}(-t)$ and $C_{1}^{*}(-t)$ matched to the Laurent pulses $C_{0}(t)$ and $C_{1}(t)$, which provide sufficient statistics for the VA decision metric. The output of each of the matched filters is sampled at every symbol interval and is given at time $t=k T$ by $z_{P}[k]=\left.\left(r(t) * C_{P}^{*}(-t)\right)\right|_{t=k T}$ (for $P=0,1$ ). The VA consists of $2 p$ states, and searches for the symbol sequence that maximizes the total path metric given that the branch metric is expressed as [28]

$$
\lambda[k]=\Re\left\{z_{0}[k] \tilde{b}_{0}^{*}[k]+z_{1}[k] \tilde{b}_{1}^{*}[k]\right\}
$$

where $z_{0}[k]$ and $z_{1}[k]$ are the sampled outputs of the matched filters to $C_{0}(t)$ and $C_{1}(t)$, respectively, and $\tilde{b}_{0}[k]$ and $\tilde{b}_{1}[k]$ are the trial complex symbols corresponding to the trial sequence of binary symbols $\tilde{a}$. This metric is used to search the trellis by performing MLSD and outputing the maximum-likelihood symbol sequence as described in Section 2.2.1. Comparing this receiver to the optimum receiver described in Section 2.2.1, we notice that in case of Bluetooth GFSK signals, the number of filters is reduced from 8 to 
2 and the number of states required in the VA is reduced in case of odd $m$ from $4 p$ to $2 p$. Moreover, the complexity of this receiver may be easily reduced by approximating the CPM signal using fewer Laurent pulses, as shown in [28] for binary CPM and in [10] for multilevel CPM. However, the resulting performance would of course be suboptimum. Regardless, the number of trellis states are still dependent on the modulation index $h$, and coherent detection is assumed. Hence, this approach is not a feasible solution for the Bluetooth receiver design, and, therefore, will not be pursued in the present work.

\subsubsection{Main Pulse}

For reduced-complexity receivers, it is desirable to develop a linear representation of a CPM signal using only one pulse. $\dot{C}_{0}(t)$ is the most important component in $(3.10)$, for it has the longest duration and contains most of the energy of the signal (as was illustrated in Fig. 3.1). Laurent used this fact to derive a 'main pulse', $P(t)$, which has the same duration as $C_{0}(t)$, and presents by itself the best approximation of $s(t)$ as

$$
\hat{s}(t)=\sum_{k=-\infty}^{+\infty} b[k] P(t-k T)
$$

where

$$
b[k]=b_{0}[k]=\exp \left(j \pi h \sum_{l=-\infty}^{k} a[l]\right)
$$

may be represented as differentially-encoded complex symbols

$$
b[k]=b[k-1] \exp (j \pi h a[k])
$$

as previously mentioned. The desired main pulse $P(t)$ is obtained through an optimization criterion which minimizes the average energy of the difference between the exact signal $s(t)$ and its approximation $\hat{s}(t) . P(\tau+m T), 0 \leq \tau \leq T$, is given by 

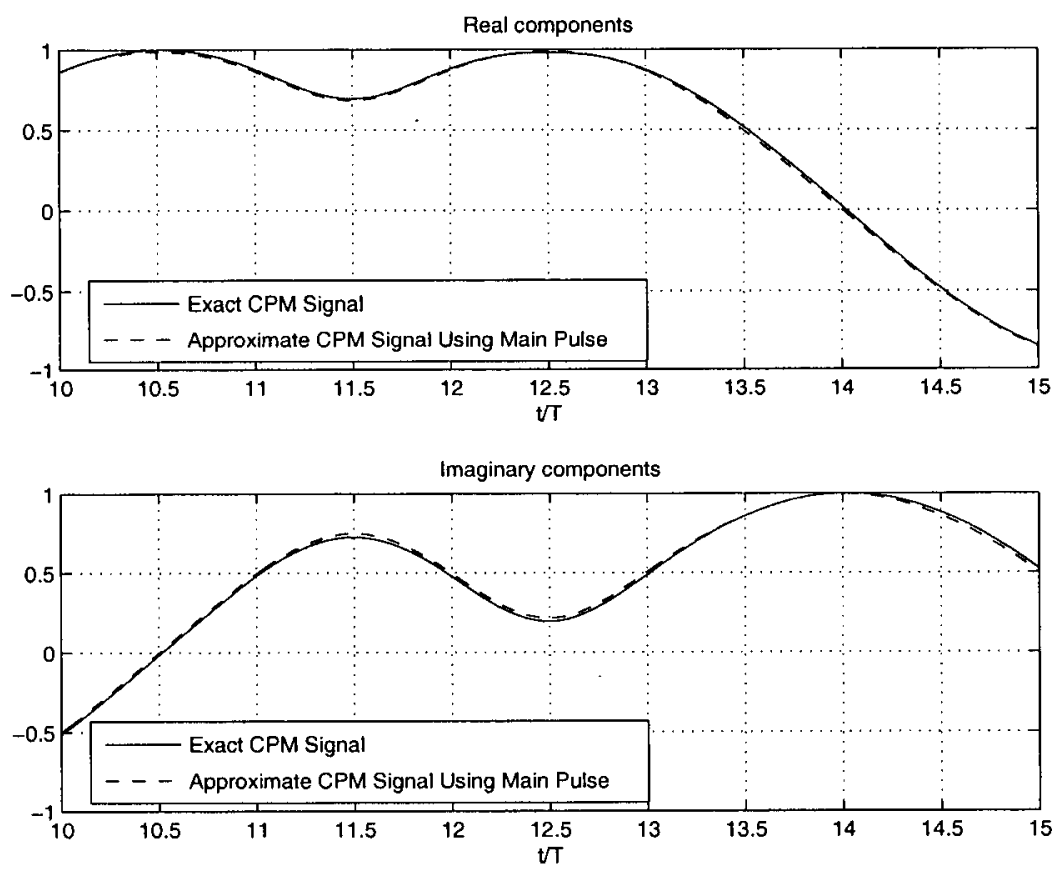

Figure 3.3: The imaginary and real components of an exact Bluetooth signal $s(t)$ and an approximate signal $\hat{s}(t)$ generated using only the main pulse $P(t)$.

$$
\begin{aligned}
P(\tau+m T)= & P_{m}(\tau) \text { with }[32] \\
P_{m}(\tau)= & \frac{1}{\sin (\pi h)} \prod_{i=-\infty}^{m-2} \cos (\varphi(\tau+i T)) \prod_{i=m+1}^{\infty} \cos (\pi h-\varphi(\tau-i T)) \\
& \cdot[\sin (\varphi(\tau+m T)) \cdot \cos (\tau+(m-1) T) \\
& -\cos (\pi h) \cdot \sin (\varphi(\tau+(m-1) T)) \cdot \cos (\pi h-\varphi(\tau+m T))] .
\end{aligned}
$$

Observing Eq. (3.18) closely, we may notice that $P(t)$ is zero for $t<0$ and $t>(L+1) T$, which proves our previous statement that $P(t)$ is the same duration. as $C_{0}(t)$. Laurent gives an alternative representation for the main pulse in [32] where it is shown to be a weighted superposition of time-shifted versions of the Laurent pulses $C_{P}(t)$. In both representations, we notice that the main pulse depends on the modulation index $h$. Hence, varying $h$ affects both the complex symbols $b[k]$ as well as the main pulse $P(t)$. Also note that the representation of CPM as given in (3.15) may be modelled as a 
phase shift keying (PSK) signal constellation of $p$ points, and the main pulse may be considered as the pulse shaping filter. Hence, the memory of the CPM manifests itself as intersymbol interference.

Figure 3.3 shows the negligible difference in the imaginary and real components of an exact Bluetooth signal as formulated in (2.9) and an approximate signal using only the main pulse as given in (3.15), both simulated for the same set of binary data, $\boldsymbol{a}$, and for $h=1 / 3$. In the following sections, we assume that the Laurent's approximation given by Eq. (3.15) holds with equality, and, thus, will be denoted hereafter by $s(t)$.

\section{$3.2 \quad$ Filter Design}

The optimum demodulator for a linear system with a pulse shaping filter $P(t)$ is known to be the matched filter $P^{*}(-t)[38]$. The noise sequence at the output of the matched filter is zero-mean Gaussian distributed noise. Although the noise variance is reduced, the noise sequence is correlated since $P(t)$ is not a $\sqrt{\text { Nyquist }}$ filter in general, and, therefore, the cascade of the main pulse and its matched filter does not satisfy the Nyquist criterion. To obtain uncorrelated additive white noise which is more convenient to deal with, a $\sqrt{\text { Nyquist }}$ receiver filter with the frequency response $H(f)$ is required such that

$$
\sum_{i=-\infty}^{+\infty}\left|H\left(f-\frac{i}{T}\right)\right|^{2}=\text { constant } .
$$

The optimum filter that satisfies this property is the whitened matched filter (WMF) [17]. The WMF is a cascade of a matched filter and a noise whitening filter. It provides sufficient statistics for the detection of the symbol sequence, and the resulting sampled noise sequence at the output of the filter is white Gaussian noise. The frequency response $H_{\mathrm{WMF}}(f)$ of the WMF corresponding to the main pulse $P(t)(3.18)$ is given 
by

$$
H_{\mathrm{WMF}}(f)=\frac{P^{*}(-f)}{\sqrt{\sum_{n=-\infty}^{+\infty}|P(f-n / T)|^{2}}}
$$

The WMF depends on the main pulse $P(t)$, which is formulated in terms of the modulation index $h$ (cf. Section 3.1.3). Hence, the WMF would ideally need to be adjusted according to the modulation index of the transmitted signal. To avoid this, and to facilitate a practical implementation, we propose replacing the WMF with the widelyused square root-raised cosine (SRC) filter. The root-raised cosine filter is a $\sqrt{\text { Nyquist }}$ filter obtained by splitting the raised cosine filter into two parts, with the frequency response $H_{\mathrm{SRC}}(f)$ of each part being the square-root of the original as [37]

$$
H_{\mathrm{SRC}}(f)= \begin{cases}1 & \text { for }|f| \leq \frac{(1-\rho)}{2 T} \\ \cos \frac{\pi T}{2 \rho}\left(|f|-\frac{(1-\rho)}{2 T}\right) & \text { for } \frac{(1-\rho)}{2 T}<|f| \leq \frac{(1+\rho)}{2 T} \\ 0 & \text { for }|f|>\frac{(1-\rho)}{2 T}\end{cases}
$$

The corresponding impulse response is given by

$$
h_{\mathrm{SRC}}(t)=\frac{4 \rho(t / T) \cos (\pi(1+\rho) t / T)+\sin (\pi(1-\rho) t / T)}{\pi t\left(1-(4 \rho t / T)^{2}\right) / T}
$$

where $\rho$ denotes the roll-off factor of the raised cosine filter. The roll-off factor $(0 \leq$ $\rho \leq 1)$ determines the excess bandwidth occupied by the signal beyond the Nyquist frequency $1 /(2 T)$.

In addition, we consider an important issue in the implementation of a finite impulse response in the following. To obtain an ideal frequency response, the impulse response has to be infinitely long. Practically, an infinite impulse response cannot be implemented, and, thus, needs to be truncated. However, truncating in the time domain leads to overshoots and ripples in the frequency domain, which degrade the performance, particularly in the presence of interference. To reduce these undesirable effects, the ideal impulse response may be multiplied by a suitable window function which 

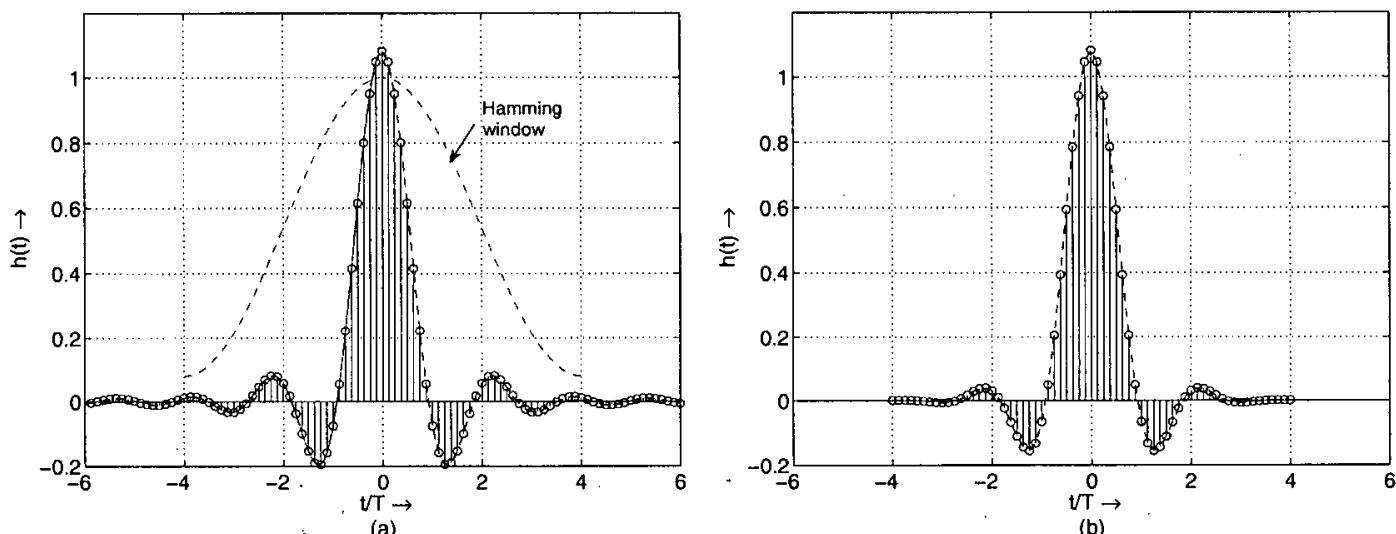

Figure 3.4: Impulse response of SRC, $h_{\mathrm{SRC}}(t)$, before Hamming window (a), and after Hamming window (b).

allows the resulting impulse response to smoothly decay to zero. Therefore, to increase interference suppression, which is mandatory for Bluetooth devices (cf. Section 2.1.5), we apply a windowing method and choose the widely used Hamming window given by

$$
w(t)= \begin{cases}0.54+0.46 \cos \left(2 \pi t / N_{H}\right) & -X_{L}\left(N_{H}\right) \leq t \leq X_{U}\left(N_{H}\right) \\ 0 & \text { elsewhere }\end{cases}
$$

where $X_{L}\left(N_{H}\right)$ and $X_{L}\left(N_{H}\right)$ represent the upper limit and lower limit of the Hamming window, respectively, and the length of the Hamming window is denoted by $N_{H}$. $X_{L}\left(N_{H}\right)=\frac{N_{H}-2}{2}$ and $X_{U}\left(N_{H}\right)=\frac{N_{H}-1}{2}$ if $N_{H}$ is odd, and $X_{L}\left(N_{H}\right)=X_{U}\left(N_{H}\right)=\frac{N_{H}}{2}$ if $N_{H}$ is even. Fig. 3.4 depicts the root-raised cosine filter in the time domain (3.22) before and after the Hamming window. The Hamming window is illustrated by the dashed curve in Fig. 3.4 (a) for a predefined value of $N_{H}$ equal to 64, which is eight times the oversampling time, and is zero otherwise.

In the following, we assume that a Hamming window with $N_{H}=64$ have been applied to both the WMF and the SRC. This requires the multiplication of the WMF and SRC filter in the time domain by the Hamming window given by (3.23). Fig. 3.5 depicts 


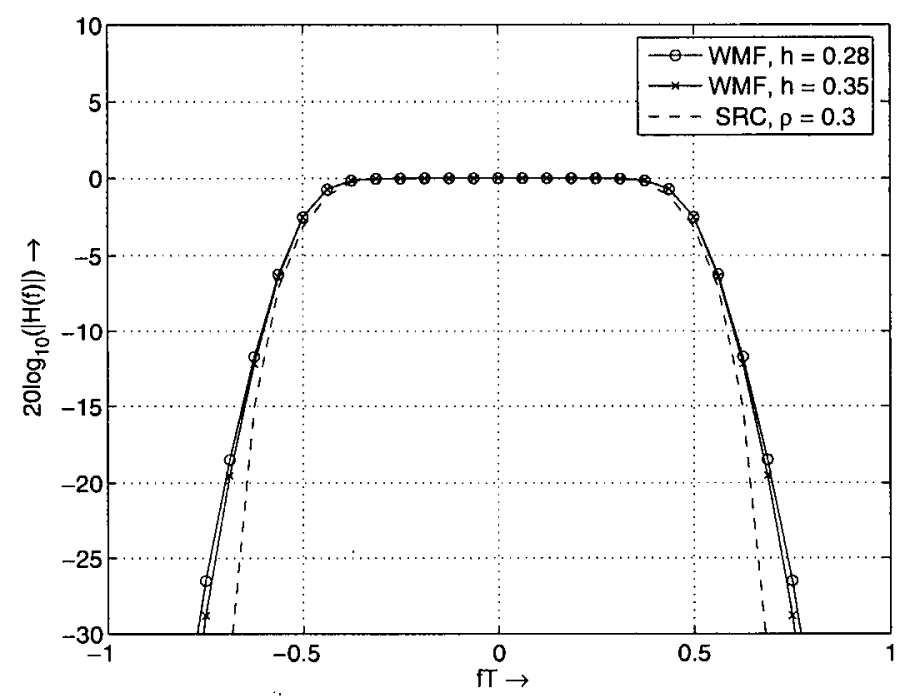

Figure 3.5: Magnitude frequency response for WMF, $H_{\mathrm{WMF}}(f)$, assuming $h=0.28$ and $h=0.35$, and for SRC filter, $H_{\mathrm{SRC}}(f)$, with $\rho=0.3$.

the magnitude frequency response of the WMF (3.20) for $h=0.28$ and $h=0.35$, respectively, and that of the $\operatorname{SRC~(3.21)~with~} \rho=0.3$. We observe that the differences of $\left|H_{\mathrm{WMF}}(f)\right|$ for different $h$ are rather small, and that the magnitude frequency response of the SRC filter well approximates that of the WMF for different $h$. Moreover, while closely resembling the WMF in the passband, the SRC also accomplishes strong outof-band interference suppression. Since the SRC filter is widely used in practice with hardware implementations available, we adopt $h_{\mathrm{SRC}}(t)$ as input filter for the proposed receiver. 


\subsection{Discrete-time Transmission Model}

The discrete-time received signal after filtering with $h_{\mathrm{SRC}}(t)$ and symbol-rate sampling can be well-approximated by

$$
\begin{aligned}
r[k] & =\left.r(t) * h_{\mathrm{SRC}}(t)\right|_{t=k T} \\
& =\mathrm{e}^{j \phi[k]} \sum_{l=0}^{q_{h}} h_{c}[l] b[k-l]+i[k]+n[k]
\end{aligned}
$$

with the discrete-time channel impulse response (CIR)

$$
h_{c}[k]=\left.P(t) * h_{\mathrm{SRC}}(t)\right|_{t=k T}
$$

of order $q_{h} . n[k]$ is the sampled noise signal, which is still AWGN, and $i[k]$ represents the sampled interference signal. Furthermore, the time variance of the phase $\phi(t)$ is assumed to be slow compared to the modulation interval $T$, and, thus, $\phi[k]=\left.\phi(t)\right|_{t=k T}$.

Through Laurent's representation (3.15), the memory of the CPM manifests itself as intersymbol interference (ISI), and $h_{c}[k]$ is referred to hereafter as the ISI channel, which may be described by a finite state machine with a trellis diagram. Recalling that the complex symbols $b[k]$ belong to a $p$-ary PSK constellation (3.16), and since the ISI channel was found to be of length $q_{h}+1$, the trellis would consist of $p^{q_{h}+1}$ states (since $\sum_{l=0}^{q_{h}} h_{c}[l] \tilde{b}[k-l]$ can assume $p^{q_{h}+1}$ different values) determined by the state vector

$$
\tilde{b}[k]=\left[\tilde{b}[k], \tilde{b}[k-1], \ldots, \tilde{b}\left[k-q_{h}\right]\right]
$$

of hypothetical (or trial) symbols $\tilde{b}[k]$. Assuming the channel phase $\phi[k]$ is known at the receiver, the VA searches for the symbol sequence which minimizes the path metric given that the branch metric at time $k$ is expressed by

$$
\begin{aligned}
\lambda[k] & =\left|\mathrm{e}^{-j \phi[k]} r[k]-\sum_{l=0}^{q_{h}} h_{c}[l] \tilde{b}[k-l]\right|^{2} \\
& =\left|\mathrm{e}^{-j \phi[k]} r[k]-\tilde{y}[k]\right|^{2}
\end{aligned}
$$




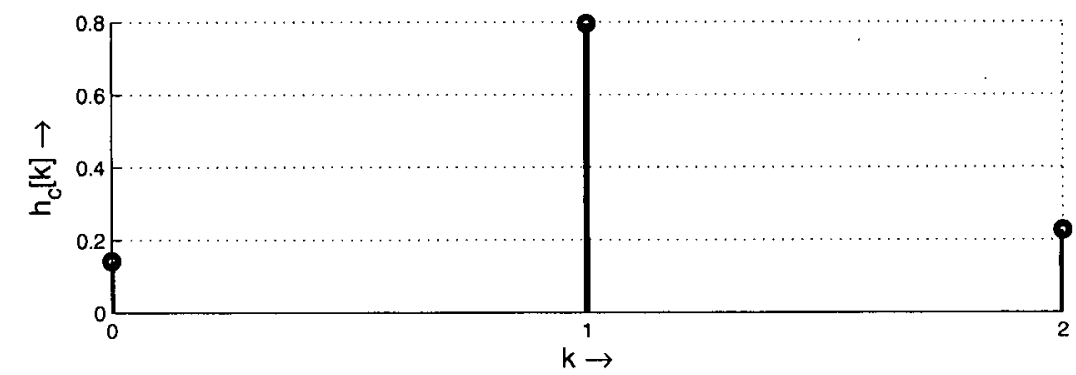

(a)

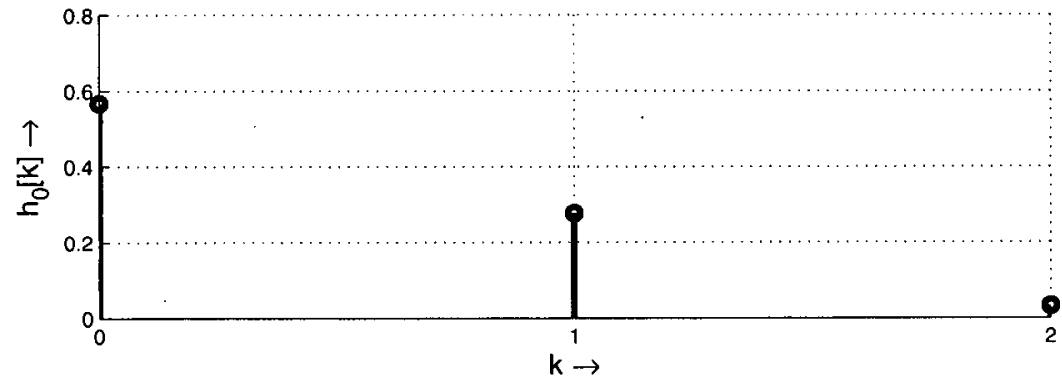

(b)

Figure 3.6: The CIR of the (a) ISI channel $h_{c}[k]$ compared to (b) its minimum phase equivalent $h_{0}[k]$.

which may be easily derived from (2.20) and represents the Euclidean distance between the received signal $\mathrm{e}^{-j \phi[k]} r[k]$ and the hypothetical transmitted signal $\tilde{y}[k]$. Observing Fig. 3.6 (a) which depicts the significant taps of the overall channel $h_{c}[k](3.26)$, we can see that $h_{c}[k]$ is of order $q_{h}=2$. Taking into account that the appropriate $p$ for modulation indices $0.28 \leq h \leq 0.35$ can be significantly larger than 8 (cf. Table 2.2), the complexity of MLSD becomes prohibitive. Hence, state reduction is mandatory, and will be employed in the present work using the concepts of reduced-state sequence estimation (RSSE) [16] and per-survivor processing [39].

\subsection{Reduced-State Sequence Estimation}

Reduced-state sequence estimation (RSSE) [15], [16] is an increasingly popular technique used to perform sequence estimation with a reduced-complexity VA. It provides a good tradeoff between performance and complexity by combining the high perfor- 
mance of MLSD with the low complexity of DFE. It truncates the number of states searched by the VA by determining the additional information no longer present in the current state from previous decisions. Moreover, it provides flexibility through a design parameter $n_{s}$ that controls the number of states in the VA, and, as a result, the complexity.

In Bluetooth, and due to the varying modulation index, it is essential to achieve state reduction independent of the modulation index $h=m / p$ with potentially large $p$ (cf. Table 2.2). As observed in the previous section, the state vector of the trellis is defined in terms of the complex symbols $b[k]$, which are expressed as (3.16), and are therefore dependent on $p$. To tackle this problem, we define a trellis state vector reduced to a certain number $n_{s}$ of hypothetical data symbols $\tilde{a}[k]$ as

$$
\tilde{\boldsymbol{a}}[k]=\left[\tilde{a}[k] \ldots \tilde{a}\left[k-n_{s}+1\right]\right] .
$$

Therefore, the number of states required for the VA is reduced to $2^{n_{s}}$, which is independent of $p$. A vector $\hat{b}[k]=\left[\hat{b}\left[k-n_{s}\right] \ldots \hat{b}\left[k-q_{h}+1\right]\right]$ of estimated symbols $\hat{b}[k]$ is associated with each state $\left(\hat{\boldsymbol{b}}[k]=\left[\hat{b}\left[k-n_{s}\right]\right]\right.$ if $\left.n_{s} \geq q_{h}\right)$. The symbols $\hat{b}[k]$ are determined by applying per-survivor processing [39], and are calculated as (3.17). The RSSE simply involves partitioning the ISI channel at each time $k$ into two using the parameter $n_{s}$, such that one part is associated with the hypothetical data symbols $\tilde{\boldsymbol{a}}[k]$ and the other retrieved from the survivor sequence as

$$
y[k]=\sum_{l=0}^{n_{s}} h_{c}[l] \tilde{b}[k-l]+\sum_{l=n_{s}+1}^{q_{h}} h_{c}[l] \hat{b}[k-l]
$$

where

$$
\tilde{b}[k-l]=\hat{b}\left[k-n_{s}-1\right] \exp \left(j \pi h \sum_{n=k-n_{s}}^{k-l} \tilde{a}[n]\right) .
$$

In other words, a state at time $k$ does not carry all the necessary symbols, and the remaining $\left(q_{h}-n_{s}\right)$ required symbols $\hat{b}[k]$ are determined from the surviving sequence. 
A constant channel phase will be assumed here, but this constraint will be relaxed in the following sections. The VA branch metric at time $k$ may be developed by applying RSSE in (3.27), resulting in

$$
\lambda[k]=\left|d[k]-\sum_{l=0}^{n_{s}} h_{c}[l] \tilde{b}[k-l]\right|^{2},
$$

where

$$
d[k]=\mathrm{e}^{-j \phi[k]} r[k]-\sum_{l=n_{s}+1}^{g_{h}} h_{c}[l] \hat{b}[k-l] .
$$

The RSSE branch metric (3.31) is controlled by the design parameter $n_{s}$ and the state trellis (3.28) is independent of $p$. It allows the equalizer to vary from a full VA $\left(n_{s} \geq q_{h}\right)$ to the simplest case of a DFE for $n_{s}=0$. The DFE structure will be illustrated in the following section.

\subsection{Decision-Feedback Equalizer Structure}

The decision-feedback equalizer (DFE), which may be interpreted as a one-state VA, is the simplest nonlinear equalizer that constitutes an attractive compromise between complexity and performance. The main idea behind the DFE is using the previous decisions to cancel ISI in the present symbol, a concept originally proposed by Austin [3] in 1967. The decision-feedback equalizer structure was fully developed by Monsen [36], of which a block diagram is shown in Fig. 3.7.

Referring to the aforementioned block diagram, the received signal vector $r$ is fed into a feedforward filter $(\mathrm{FFF}) \boldsymbol{f}_{F}=\left[f_{F}[0], f_{F}[1], \ldots, f_{F}\left[q_{F}\right]\right]$ of order $q_{F}$ which suppresses the contribution of the precursor ISI. To cancel the ISI in the present symbol, the feedback filter $(\mathrm{FBF}) \boldsymbol{f}_{B}=\left[f_{B}[0], f_{B}[1], \ldots, f_{B}\left[q_{B}\right]\right]$ of order $q_{B}$ uses previously-detected symbols, and the output of the FBF is then subtracted from the output of the FFF. Assuming correct previous decisions, the interference from previously detected symbols is removed 


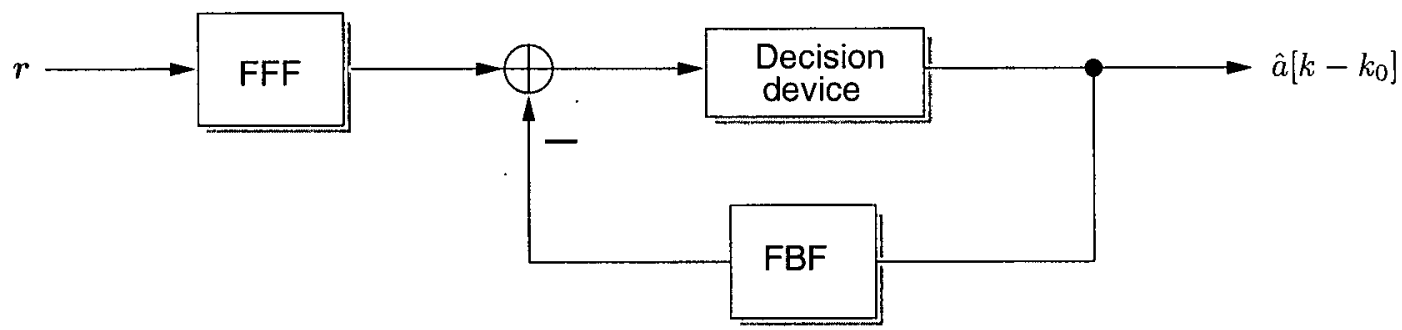

Figure 3.7: Block diagram of decision-feedback equalizer.

through the FBF, and the constant $k_{0}$, known as the decision delay, specifies the number of future measurements processed before any decision is made on the present symbol. The input $z[k]$ to the decision device may be expressed $\mathrm{as}^{2}$

$$
z[k]=\sum_{l=0}^{q_{F}} f_{F}[l] r[k-l]-\sum_{l=1}^{q_{B}} f_{B}[l] \hat{I}\left[k-l-k_{0}\right]-f_{B}[0] \tilde{I}\left[k-k_{0}\right]
$$

where $\hat{\boldsymbol{I}}$ is the vector of previously estimated symbols. Since the DFE operates under the assumption that the past decisions are correct, which is almost always violated, the DFE is evidently a suboptimum equalization scheme.

The coefficients of the FFF and FBF filters obviously have a great impact on the DFE performance. Several techniques for computing the FFF and FBF coefficients were proposed in literature, of which a comprehensive review was given in [7]. In the present work, we adopt the simple DFE structure considering both coherent and noncoherent detection and compute the coefficients of the corresponding filters as outlined in the next section.

\subsubsection{Minimum Phase Channel}

A minimum-phase overall impulse response is essential to obtain a high performance with RSSE [16], especially in the extreme case of RSSE, DFE. The finite impulse

\footnotetext{
${ }^{2}$ Usually a threshold decision is made, where the input to the decision device does not include the last term on the right hand side of (3.33).
} 
response (FIR) transfer function $H_{\min }(z)$ of a minimum phase channel is such that $H_{\min }(z)$ has roots only inside the unit circle. A minimum phase channel $h_{\min }[k]$ has the fastest decay, ensuring that the energy concentration in the first samples is maximized

$$
\sum_{l=0}^{l_{h}-1}\left|h_{\min }[k]\right|^{2} \geq \sum_{l=0}^{l_{h}-1}|h[k]|^{2}
$$

where the $h_{\min }[k]$ is the minimum phase equivalent of $h[k]$ and $l_{h}$ is the length of the CIR. Since the decision. in a DFE largely depends on the first tap (3.33), it is obvious that a minimum-phase channel is desirable. Ideally, a minimum phase channel is produced by applying a discrete-time prefilter $f[k]$ with an allpass characteristic to the sampled received signal $r[k]$. The minimum phase equivalent of the Bluetooth ISI channel $h_{c}[k]$ (3.26) is obtained by prefiltering it by $f[k]$ as

$$
h_{c}[k] * f[k],
$$

where the minimum phase equivalent, $h_{0}[k]$, represents the last $q_{h}+1$ taps of the resulting impulse response. The allpass filter $f[k]$ alters the phase without affecting the overall magnitude response, producing an AWGN noise sequence at the output. In order not to change the magnitude frequency response, the all-pass filter moves the roots of the FIR transfer function $H_{c}(z)$ of $h_{c}[k]$ which are outside the unit circle simply by reflecting them about the unit circle, resulting in the roots of its corresponding minimum phase $H_{0}(z)$, as illustrated in Fig. 3.8.

For a practical implementation, a finite-impulse response (FIR) approximation of the allpass filter is of interest. A comprehensive review of the state-of-the-art prefiltering strategies is given in [20]. In this research work, the computation of the prefilter is based on linear prediction, as was presented in [20]. This prefilter consists of the cascade of a matched filter to the channel and a prediction error filter, which is calculated via the Levinson-Durbin algorithm. It was found to be an efficient prefiltering method for the ISI channel $h_{c}[k]$ in addition to having low computational complexity. The prefilter 


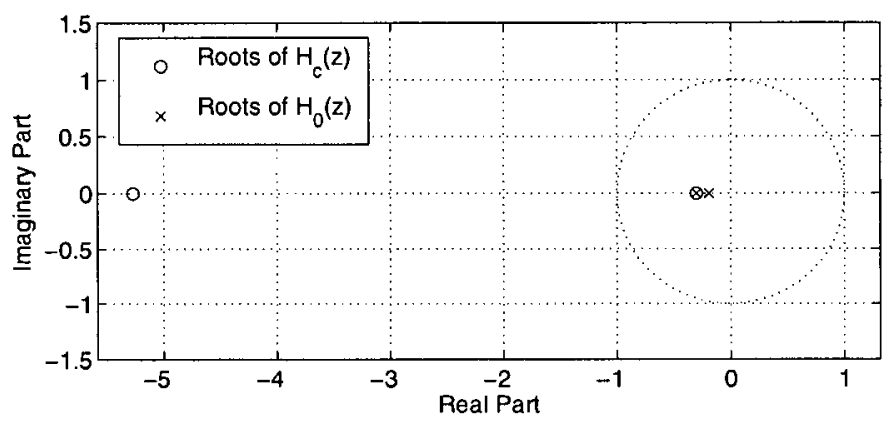

Figure 3.8: The roots of $H_{c}(z)$ compared to the roots of its minimum phase equivalent $H_{0}(z)$.

is designed to be of order $q_{F}=6$, and was found to slightly vary with $h$, but only insignificant performance differences incur if it is designed for a fixed $h$. The overall channel $h_{0}[k]$ is of order $q_{B}=2$ since it is of the same length as $h_{c}[k]$, as was mentioned earlier, and is shown for a fixed $h=1 / 3$ in Fig. 3.6 (b).

\subsubsection{DFE Decision Rule}

From the previous discussion, it is clear that in a DFE structure, $f[k]$ would serve as an effective FFF, while $h_{0}[k]$ would be its corresponding FBF, and, hence, in the present work, we employ $f[k]$ as the FFF and $h_{0}[k]$ as the FBF in the proposed DFE structure. To further illustrate the role of the FBF in reducing the effect of the ISI caused by previous symbols, we may rewrite $r[k] * f[k]$ as

$$
r[k] * f[k]=\mathrm{e}^{j \phi[k]} h_{0}[0]\left(b[k]+\frac{1}{h_{0}[0]} \sum_{l=1}^{q_{B}} h_{0}[l] b[k-l]\right)+i[k] * f[k]+n[k] * f[k]
$$

where the term

$$
\sum_{l=1}^{q_{B}} h_{0}[l] b[k-l]
$$

represents the intersymbol interference. Assuming coherent detection, the DFE deci- 
sion rule reads

$$
\hat{a}\left[k-k_{0}\right]=\underset{\tilde{a}\left[k-k_{0}\right]}{\operatorname{argmax}}\left\{\Re\left\{d_{\mathrm{DFE}}^{*}[k] h_{0}[0] \tilde{b}\left[k-k_{0}\right]\right\}\right\}
$$

where

$$
d_{\mathrm{DFE}}[k]=\mathrm{e}^{-j \phi[k]} r[k] * f[k]-\sum_{l=1}^{q_{B}} h_{0}[l] \hat{b}\left[k-k_{0}-l\right] .
$$

We may notice that the FBF subtracts out the ISI term from the decision. This decision rule corresponds to the branch metric (3.31) with $n_{s}=0$. We have to note here that for the proposed receiver the ISI channel is prefiltered to obtain its minimum phase equivalent only in case of DFE although, theoretically, a minimum phase channel is essential for RSSE as well. However, since the ISI channel $h_{c}[k]$ only has 3 significant taps, a 4-state RSSE is interpreted as full state VA, and a 2-state RSSE is not severely affected if the channel is not minimum phase. Consequently, the all-pass filter $f[k]$ is only employed in case of DFE.

\subsection{Noncoherent Detection}

So far, we have successfully employed coherent detection using RSSE and DFE, and the results presented in Chapter 4 show remarkable performance results. However, Bluetooth signals encounter channel phase variations due to oscillator phase noise and frequency instabilities of Bluetooth transceivers (cf. Section 2.1.5), and, as a result, coherent detection which assumes reliable phase estimates $\hat{\phi}[k]$ of $\phi[k]$ is not possible in practice. Noncoherent equalization algorithms based on sequence detection were proposed in [19] and [11]. These schemes are power efficient and highly robust against channel phase variations, for the channel phase is implicitly estimated. The optimum noncoherent MLSD branch metric is given at time $k$ by [11]

$$
\lambda[k]=\sum_{v=0}^{k}\left(|r[v]|^{2}+|\tilde{y}[v]|^{2}\right)-2 \sum_{v=0}^{k}\left|r[v] \tilde{y}^{*}[v]\right| .
$$

Evidently, this branch metric has unlimited memory, for at each time $k$ it depends on the entire previous sequence of symbols. To limit the computational complexity, 
Colavolpe and Raheli [11] proposed reducing the branch metric memory by truncating the window of observations to a finite value $N, N \geq 2$. Hence, (3.39) becomes

$$
\lambda[k]=\sum_{v=k-N+1}^{k}|\tilde{y}[v]|^{2}-2 \sum_{v=k-N+1}^{k}\left|r[v] \tilde{y}^{*}[v]\right| .
$$

The term $|r[v]|^{2}$ was eliminated in (3.40) since it is independent of the decision. A noncoherent DFE (NDFE) was further derived from (3.40) in [45], with the branch metric, now suboptimum, given by

$$
\lambda_{\mathrm{NDFE}}[k]=\sum_{v=k-N+1}^{k}\left|y_{\mathrm{DFE}}[v]\right|^{2}-2 \sum_{v=k-N+1}^{k}\left|r_{\mathrm{DFE}}[v] y_{\mathrm{DFE}}^{*}[v]\right|
$$

where $r_{\mathrm{DFE}}[k]$ is the output of the FFF and $y_{\mathrm{DFE}}[k]$ is the output of the FBF. Using the FBF and FFF recommended in Section $3.5, r_{\mathrm{DFE}}[k]$ and $y_{\mathrm{DFE}}[k]$ at time $k$ can be formulated as

$$
r_{\mathrm{DFE}}[k]=\sum_{l=0}^{q_{F}} f[l] r[k-l]
$$

and

$$
y_{\mathrm{DFE}}[k]=\sum_{l=0}^{q_{B}} h_{0}[l] b\left[k-k_{0}-l\right]
$$

where the complex symbols $b\left[k-k_{0}\right]$ are calculated as (3.17). Hence, (3.41) becomes

$$
\begin{aligned}
\lambda_{\mathrm{NDFE}}[k]= & \sum_{v=k-N+1}^{k-1}\left|\hat{y}_{\mathrm{DFE}}[v]\right|^{2}+\left|\sum_{l=1}^{q_{B}} h_{0}[l] \hat{b}\left[k-k_{0}-l\right]+h_{0}[0] \tilde{b}\left[k-k_{0}\right]\right|^{2} \\
& -2\left|\hat{q}_{\mathrm{ref}}^{N}[k-1]+r_{\mathrm{DFE}}[k] \hat{y}_{\mathrm{DFE}}^{*}[k]\right|
\end{aligned}
$$

where

$$
\hat{q}_{\mathrm{ref}}^{N}[k-1]=\sum_{v=k-N+1}^{k-1} r_{\mathrm{DFE}}[v] \hat{y}_{\mathrm{DFE}}^{*}[v]
$$

Here, $\hat{y}_{\mathrm{DFE}}[k]=\sum_{l=1}^{q_{B}} h_{0}[l] \hat{b}\left[k-k_{0}-l\right]+h_{0}[0] \tilde{b}\left[k-k_{0}\right], \hat{b}\left[k-k_{0}-l\right]$ represent the previouslydetected symbols, while $\tilde{b}\left[k-k_{0}\right]$ is the hypothetical complex symbol. The phase of $\hat{q}_{\mathrm{ref}}^{N}[k-1]$ constitutes the estimate of the phase distortion caused by the channel, where the number of phase reference contributions is controlled by the finite value $N$, and, 
therefore, $\hat{q}_{\text {ref }}^{N}[k-1]$ is referred to as the $N$-phase reference. For a time-invariant channel phase, the greater the $N$, the less noise variance in the phase estimate, and, as a result, the better the performance. However, the computational complexity increases as well due to the increasing number of terms in (3.45). Schober and Gerstacker [44], [45], suggest avoiding this problem by recursively calculating the phase reference as

$$
\hat{q}_{\mathrm{ref}}^{\alpha}[k-1]=\alpha \hat{q}_{\mathrm{ref}}^{\alpha}[k-2]+r_{\mathrm{DFE}}[k-1] \hat{y}_{\mathrm{DFE}}^{*}[k-1]
$$

where the design parameter $\alpha, 0 \leq \alpha \leq 1$, acts as a forgetting factor, and $\hat{q}_{\mathrm{ref}}^{\alpha}[k-1]$ is referred to as the $\alpha$-phase reference. Similar to $N$, in case of constant channel phase, as $\alpha$ increases, the performance improves. However, phase jitters and frequency offsets contribute to a time-varying phase which does not represent the actual channel phase (cf. Section 2.1.5). In this case, the noise variance of the phase estimate will be high, and will accumulate as $N$ or $\alpha$ become larger, degrading the performance. Consequently, the optimum values of $N$ and $\alpha$ vary and depend on the channel parameters, and the phase jitter and frequency offset variations. Assuming constant channel phase, the performance approaches that of coherent detection as $\alpha \rightarrow 1$ and $N \rightarrow \infty$. This is an expected result since these values lead to the optimal branch metric (3.39) with no memory truncation.

In the following, the metric associated with $\hat{q}_{\mathrm{ref}}^{N}[k-1]$ is referred to as the $N$-metric, while that associated with $\hat{q}_{\text {ref }}^{\alpha}[k-1]$ is referred to as the $\alpha$-metric. The performance of the $N$ - and the $\alpha$-metric was found to be approximately equivalent, precisely according to the following equation [46]

$$
\alpha=1-\frac{2}{N}
$$

However, since the $\alpha$-metric requires less number of arithmetic operations [46], we adopt the $\alpha$-metric in the present work.

To perform noncoherent RSSE (NRSSE) using the VA, the same concept which involves estimating the channel phase is applied. However, in contrast to the NDFE, the 
previously estimated symbols are dependent upon the surviving sequence of the state being considered. The NRSSE decision rule involves minimizing the following branch metric

$$
\lambda[k]=|y[k]|^{2}-2\left|\hat{q}_{\mathrm{ref}}^{\alpha}[k-1]+r[k] y^{*}[k]\right|
$$

where

$$
\hat{q}_{\mathrm{ref}}^{\alpha}[k-1]=\alpha \hat{q}_{\mathrm{ref}}^{\alpha}[k-2]+r[k-1] y^{*}[k-1]
$$

and $y[k]$ is calculated as for coherent RSSE (3.29). Therefore, similarly, the NRSSE decision rule is controlled by the design parameter $n_{s}$. Also similar to coherent RSSE, prefiltering is not employed, as was explained in Section 3.5.2.

\subsubsection{NDFE Decision Rule}

The branch metric given in Eq. (3.44) was further developed in [45]. The limiting performance for $N \rightarrow \infty$, or, equivalently, $\alpha \rightarrow 1$, was investigated, and the obtained results were also applied to finite value of $N$ and to $\alpha<1$. The result was a modified suboptimum NDFE decision rule expressed as

$$
\begin{aligned}
\hat{b}\left[k-k_{0}\right] & =\underset{\tilde{b}\left[k-k_{0}\right]}{\operatorname{argmin}}\left\{\left|d_{\mathrm{NDFE}}[k]-h_{0}[0] \tilde{b}\left[k-k_{0}\right]\right|^{2}\right\} \\
& =\underset{\tilde{b}\left[k-k_{0}\right]}{\operatorname{argmax}}\left\{\Re\left\{d_{\mathrm{NDFE}}^{*}[k] h_{0}[0] \tilde{b}\left[k-k_{0}\right]\right\}\right\}
\end{aligned}
$$

where

$$
d_{\mathrm{NDFE}}[k]=\frac{\left(\hat{q}_{\mathrm{ref}}[k-1]\right)^{*}}{\left|\hat{q}_{\mathrm{ref}}[k-1]\right|} r_{\mathrm{DFE}}[k]-\sum_{l=1}^{q_{B}} h_{0}[l] \hat{b}\left[k-k_{0}-l\right] .
$$

$\hat{q}_{\text {ref }}[k-1]$ may be calculated using $N(3.45)$ or $\alpha$ (3.46). As mentioned earlier, in the present work, we use the $\alpha$-metric, and in the following, the NDFE receiver is the final adopted receiver design which refers to the receiver employing the decision rule given by (3.50). We may notice that this decision is similar to the coherent decision metric (see (3.32) and (3.31)) with $n_{s}=0$ (since this is a DFE), and with $\mathrm{e}^{-j \phi[k]} r[k]$ replaced by $\frac{\left(\hat{q}_{\mathrm{ref}}[k-1]\right)^{*}}{\left.\left|\hat{q}_{\mathrm{ref}}\right| k-1\right] \mid} r_{\mathrm{DFE}}[k]$. 


\subsubsection{Frequency Offset Estimation}

The NDFE decision rule presented in the previous section has proven to be extremely power efficient and robust against channel phase variations (cf. Chapter 4). However, for Bluetooth-standard compliant devices, deviations from the center frequency as large as $\Delta f T=0.1$ are admissible [51], and these contribute to additional phase variations which are not accounted for in (3.50). Variations of the phase reference which explicitly account for a frequency offset have been proposed in [12],[31]. Applied to $\hat{q}_{\text {ref }}[k-1]$ in (3.46), the modified update equation reads

$$
\hat{q}_{\mathrm{ref}}[k]=\left(\alpha \hat{q}_{\mathrm{ref}}[k-1]+r_{\mathrm{DFE}}[k] \hat{y}_{\mathrm{DFE}}^{*}[k]\right) \mathrm{e}^{j 2 \pi \widehat{\Delta f}[k-1]}
$$

where the frequency offset estimate $e^{j 2 \pi \widehat{\Delta f}[k]}=p_{\text {ref }}[k] /\left|p_{\text {ref }}[k]\right|$ follows from

$$
p_{\text {ref }}[k]=\beta p_{\text {ref }}[k-1]+r_{\mathrm{DFE}}[k] \hat{y}_{\mathrm{DFE}}^{*}[k]\left(r_{\mathrm{DFE}}[k-1] \hat{y}_{\mathrm{DFE}}^{*}[k-1]\right)^{*}
$$

Similar to $\alpha, \beta, 0 \leq \beta<1$, is a forgetting factor for the frequency offset estimation. The second term in the right hand side of Eq. (3.54) represents the estimate of the frequency offset over one symbol duration. The modified phase reference as given by (3.53) is still robust against phase variations, but now provides reliable performance in the presence of oscillator frequency offsets as well. This will be clearly illustrated in Chapter 4. The choice of $\alpha$ and $\beta$ trades performance for constant phase and frequency offset and robustness against phase variations and oscillator drifts, respectively (see the results in Chapter 4).

\subsubsection{Adaptive NDFE}

In the previous sections, we have tackled the problems of phase variations including the frequency offsets, and presented the corresponding solutions. There remains the problem of the varying modulation index $h$ which affects several parameters in the proposed receiver. Although the receiver input filter design (cf. Section 3.2) as well 
as the FBF and FFF filters (cf. Section 3.5) turned out to be almost unaffected by varying $h$, considerable deviations of the decision metrics will occur even for small mismatches between the modulation index $\hat{h}$ assumed at the receiver and the actual $h$ at the transmitter. This is evident from the formulation of the complex symbols given by (3.30): Hence, even in case of a correct estimated binary symbol $a[k]$, due to the mismatch in $h$, the estimated complex symbol will be incorrect.

We may assume a nominal value $\hat{h}$ at the receiver regardless of the modulation index's actual value $h$. However, results presented in Chapter 4, show significant performance degradation since $h$ at the transmitter is allowed to vary in a relatively large interval $(0.28 \leq h \leq 0.35)$. This is especially the case for large values of $\alpha$ and $\beta$ since the errors accumulated from previous estimates are of greater weight.

To account for this problem, we propose to test a set $\mathcal{H}$ of modulation index hypotheses $\tilde{h}$, and, after an estimation period, $N_{e}$, to select the "best" one. We calculate $|\mathcal{H}|$ NDFE accumulated path metrics, each for a total of $N_{e}$ symbols. A pragmatic approach to determine the best alternative is to decide for the $\tilde{h}$ that yields the smallest accumulated path metric. Therefore, the performance as well as the complexity of this $h$ adaptation depends on the number of hypothesis and the length of the estimation period. We employ this adaptation technique to the noncoherent decision feedback equalizer presented in the previous sections, and the resulting receiver is referred to as adaptive NDFE (ANDFE). This adaptation process is applied to the first packet received, and the estimated modulation index used to detect the rest of the symbol sequences. $\hat{h}$ is obtained by the following decision rule

$$
\hat{h}=\underset{\tilde{h} \in \mathcal{H}}{\operatorname{argmax}}\left\{\sum_{k=1}^{N_{e}} \Re\left\{d_{\mathrm{NDFE}}^{*}[k] h_{0}[0] \tilde{b}\left[k-k_{0}\right]\right\}\right\} .
$$

Clearly, the greater the set $\mathcal{H}$ and the estimation period $N_{e}$, the better the performance of ANDFE. From the results given in Chapter 4, we may deduce that four modulation index hypotheses and estimation over 50 symbol intervals is quite sufficient to obtain 


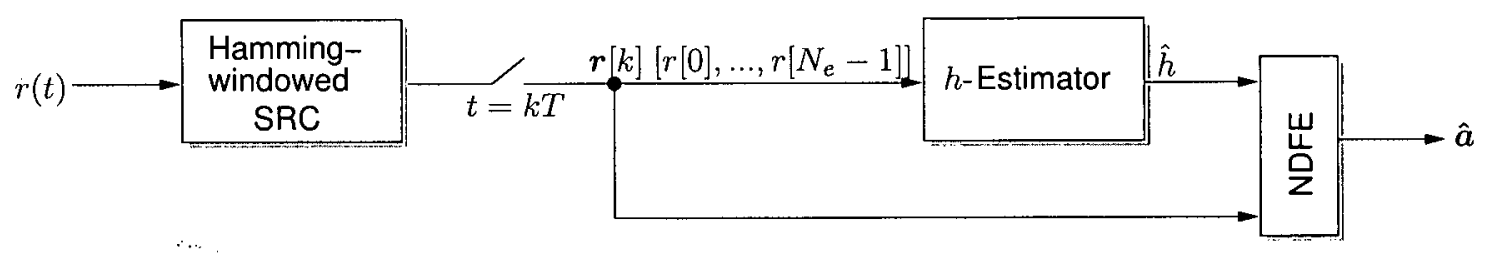

Figure 3.9: Structure of the proposed Bluetooth receiver.

a performance close to the case of $h$ being perfectly known to the receiver. With these parameters, the complexity increase of ANDFE compared to NDFE is negligible, but results show significant performance improvements under varying $h$ conditions.

\subsection{Summary of Proposed Receiver Structure}

The structure of the Bluetooth receiver designed in the present work is shown in Fig. 3.9. The proposed receiver is based on Laurent's decomposition which presents the CPM signal as a superposition of PAM pulses (3.10). We adopt the approximation which expresses the Bluetooth GFSK signal in terms. of one 'main pulse'. The recommended receiver input filter is the SRC filter (3.21), which is a practical choice that highly resembles the optimal WMF (3.20). To increase interference suppression, a Hamming window (3.23) is applied to the SRC filter. The output of the filter is symbol period sampled and fed into a simple noncoherent decision-feedback equalizer, through which phase estimation is employed as given in (3.51). Furthermore, for robust detection in the presence of high frequency offsets, frequency offset estimation is employed using (3.53). Moreover, due to the varying modulation index $(0.28 \leq h \leq 0.35)$ in Bluetooth systems, we propose using ANDFE (3.55), where an estimate of the modulation index of the transmitted signal is given. The chosen modulation index is the one which maximizes the path metric over a limited number of samples $N_{e},\left(25 \leq N_{e} \leq 50\right)$, and is selected from a predefined set of hypotheses, $\mathcal{H},(|\mathcal{H}|=2,4)$. This estimated $\hat{h}$ is then used to detect the rest of the received signal, and the final output is the sequence 
of estimated binary symbols $\hat{\boldsymbol{a}}$. 


\section{Chapter 4}

\section{Performance Results and}

\section{Discussion}

This chapter presents and analyzes the performance results of the designed Bluetooth receiver described in Chapter 3, which was simulated using $\mathrm{C}++$ and Matlab. We assume the channel model is as given by (2.13), and perform detection for a block of 1500 data points at one time. These blocks are generated until a minimum of 1000 errors is obtained, giving a high level of accuracy. To put the results into context, the proposed receiver is compared to that of the NSD and the LDI, which are considered relevant benchmarks, as discussed in Section 2.2.2. Furthermore, we consider the MLSD bound (cf. Section 2.3) as the theoretical performance limit [2]. The results for the LDI and NSD are obtained from [27], and the MLSD bound is calculated in the present work for different values of $h$. When comparing the performance of different receivers, we measure the signal-to-noise ratio required to achieve a BER of $10^{-3}$ since this is the required BER for Bluetooth receivers [51].

Different performance results were observed when the sampling start point was changed. Therefore, when obtaining the discrete-time channel model (3.25), we optimize our sampling start point to get the best performance possible using the adopted SRC filter. We 
use an oversampling time of 8 , and the total length of the employed Hamming window, and as a result, the receiver input filter, is given by $8 T$.

The first section of this chapter shows the coherent detection performance results for RSSE and DFE. In case of DFE, the FFF and FBF are of orders $q_{F}=6$ and $q_{B}=2$ (cf. Section 3.5.1), and are computed for a fixed modulation index $h=1 / 3$ throughout the chapter. Moreover, the modulation index of the transmitted signal is also assumed to be $1 / 3$ and known at the receiver and the receiver input filter is the SRC filter, unless stated otherwise. The second section is devoted to noncoherent detection were the channel phase is implicitly estimated in various scenarios.

Most of the presented figures investigate the bit error rate (BER) performance as a function of the signal-to-noise ratio (SNR) $E_{b} / N_{0}$ in decibels $(\mathrm{dB})$, where $E_{b}$ is the received energy per bit and $N_{0}$ is the one-sided noise power spectral density of the underlying passband noise process. In other cases, the required SNR for BER $=10^{-3}$, which is representative in case of Bluetooth devices [51], is recorded for different values of the modulation index $h$.

\subsection{Coherent Detection}

In this section, we present the results obtained for coherent detection; i.e, assuming a perfectly estimated channel phase at the receiver. These serve as the basis for our filter selection and our evaluation of the performance loss which the proposed receiver suffers as a result of state reduction.

\subsubsection{Filter Selection}

We have selected the SRC filter as the input receiver filter for the proposed receiver. The similarity in the magnitude frequency responses for the WMF and SRC filters was shown in Chapter 3. Furthermore, in this section, to support the decision of selecting 


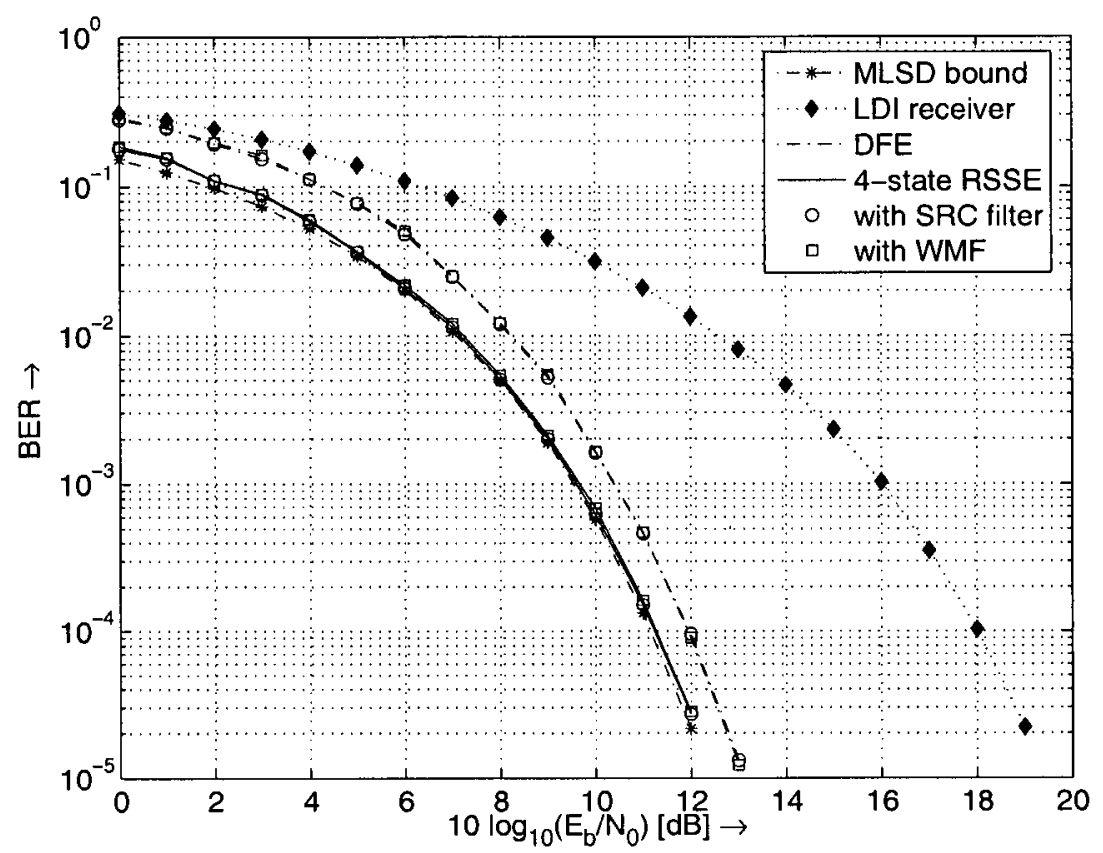

Figure 4.1: Performance comparison of the SRC and WMF as receiver filters. Coherent detection with $h=1 / 3$ is assumed.

the SRC filter, we demonstrate the performance of the SRC filter [Eq. (3.21)] and compare it with that of the optimal WMF [Eq. (3.20)]. In Fig. 4.1, we show the BER as a function of the SNR for RSSE with 4 states and for DFE, using, in both cases, the WMF and the SRC, respectively. For comparison, the BER curves for the MLSD bound and the LDI receiver are also included.

We notice that for the 4-state RSSE, as well as the DFE, the curves for the SRC filter almost coincide with those of the WMF. As a matter of fact, a closer observation would reveal that in case of a 4 -state $\mathrm{RSSE}$, there is $\simeq 0.05 \mathrm{~dB}$ performance improvement when the $\mathrm{SRC}$ is used, while in case of a DFE, there is $\simeq 0.01 \mathrm{~dB}$ performance improvement when the WMF is used. Ideally, of course, the optimal WMF filter would generate better performance in case of the full-state (4-state) RSSE. However, due to the fact that the sampling time is optimized for the SRC filter, as was previously mentioned, this is not the case here. Regardless, the performance differences are almost negligible. 


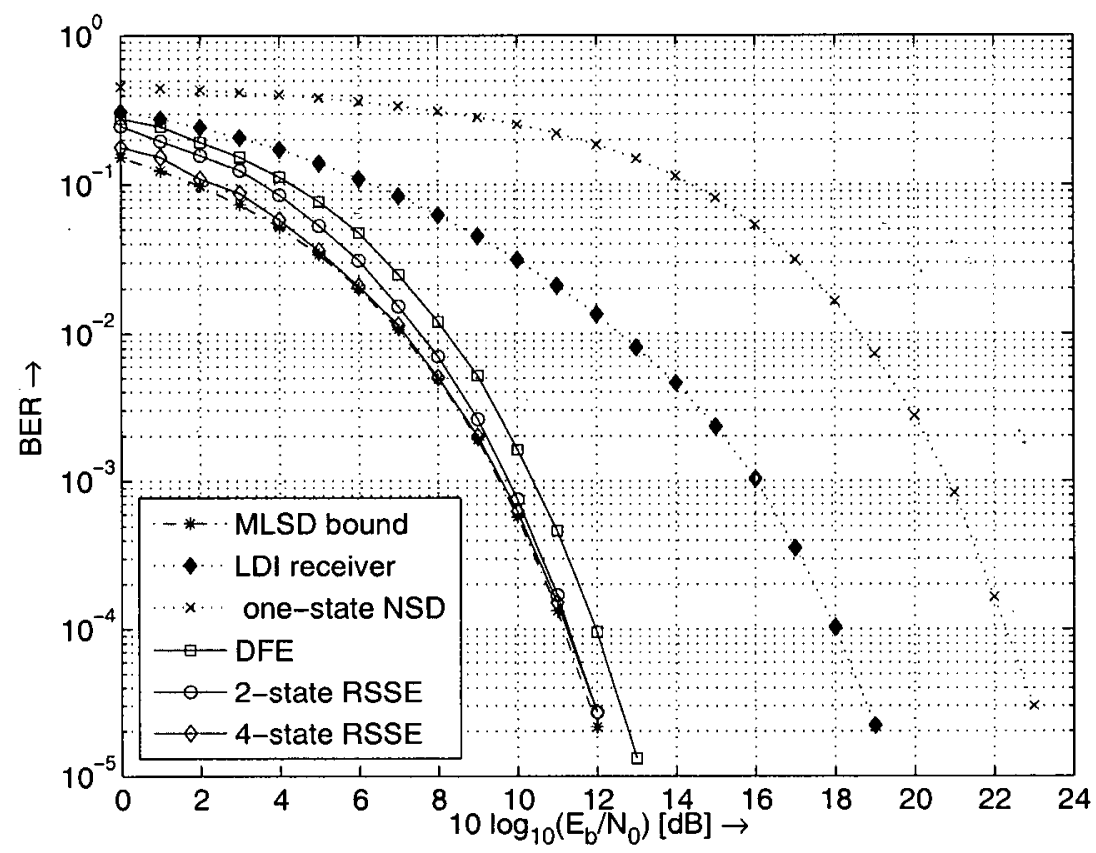

Figure 4.2: Evaluation of the state reduction in the proposed receiver. Coherent detection with $h=1 / 3$ is assumed.

Therefore, the proposed SRC filter is a practical and ideal choice for the input receiver filter, and will be used for the following simulations unless states otherwise.

\subsubsection{State Reduction}

Observing the BER curves in Fig. 4.2 for 2-state RSSE [Eqs. (3.31)-(3.32)], 4-state RSSE and DFE [Eqs. (3.37)-(3.38)], all using the SRC filter, and comparing them to the LDI, MLSD, and one-state NSD, we make the following notes. First, the RSSE with only two states approaches very closely the MLSD bound. Second, the DFE incurs a loss of $\simeq 0.7 \mathrm{~dB}$ compared to the 4-state RSSE, and less than $0.6 \mathrm{~dB}$ compared to the 2state RSSE, which is highly acceptable considering the reduction in complexity. Third, comparing the performance of the proposed receiver and NSD using a one-state trellis (or DFE), we notice tremendous differences with the NSD suffering a performance loss of more than $10 \mathrm{~dB}$. The proposed receiver, on the other hand, outperforms the LDI by more than $5 \mathrm{~dB}$, which is evidently a remarkable result using a one-state trellis. 


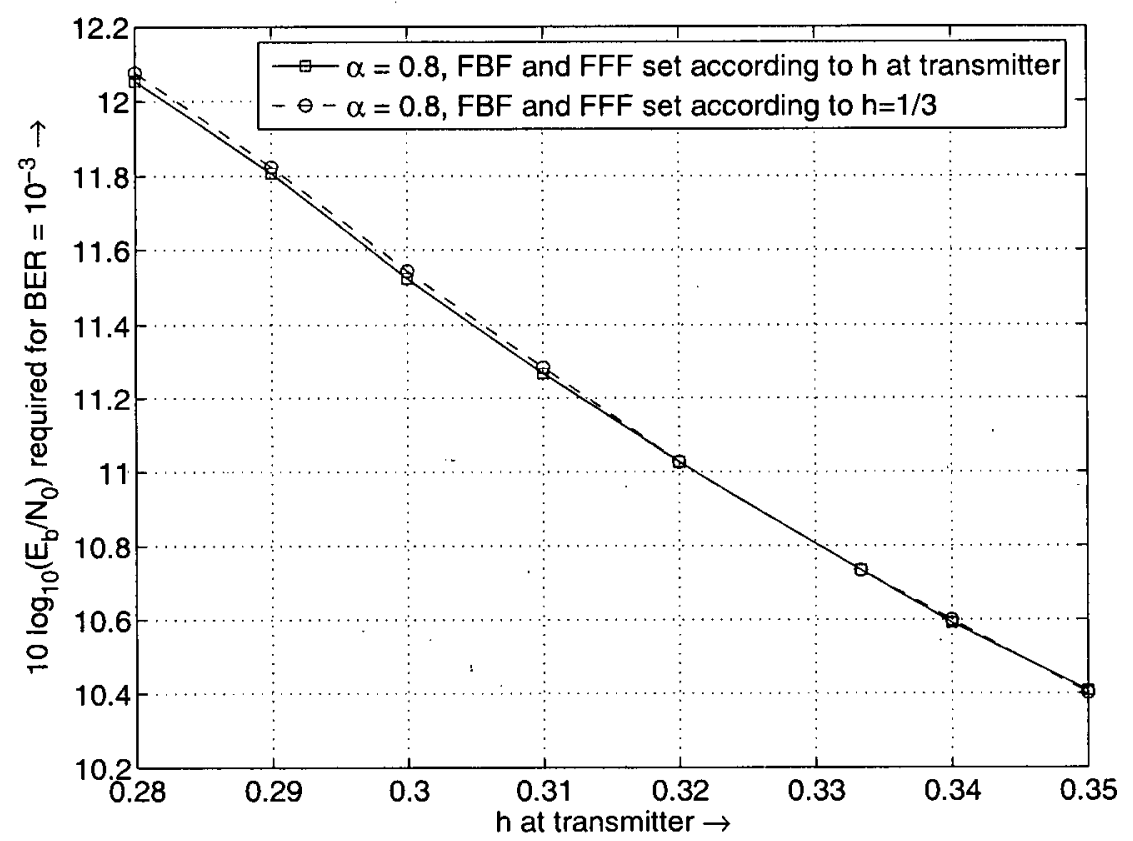

Figure 4.3: Performance of NDFE with $\alpha=0.8$ with FBF and FFF fixed at the receiver, or varying according to the value of $h$ at the transmitter.

Similar results are expected in case of noncoherent DFE, which will be considered in the following sections.

\subsection{Noncoherent Detection}

In this section, we evaluate the performance of the proposed receiver in case of noncoherent detection, where the channel phase is estimated using the phase reference. We briefly discuss and demonstrate the performance of NRSSE [Eqs. (3.48)-(3.49)], and focus on the performance of NDFE [Eqs. (3.51)-(3.52)], which constitutes the final receiver design (cf. Section 3.7). The results for MLSD, LDI, and coherent DFE are included for comparison purposes. The modulation index $h=1 / 3$ and is known at the receiver unless stated otherwise. 


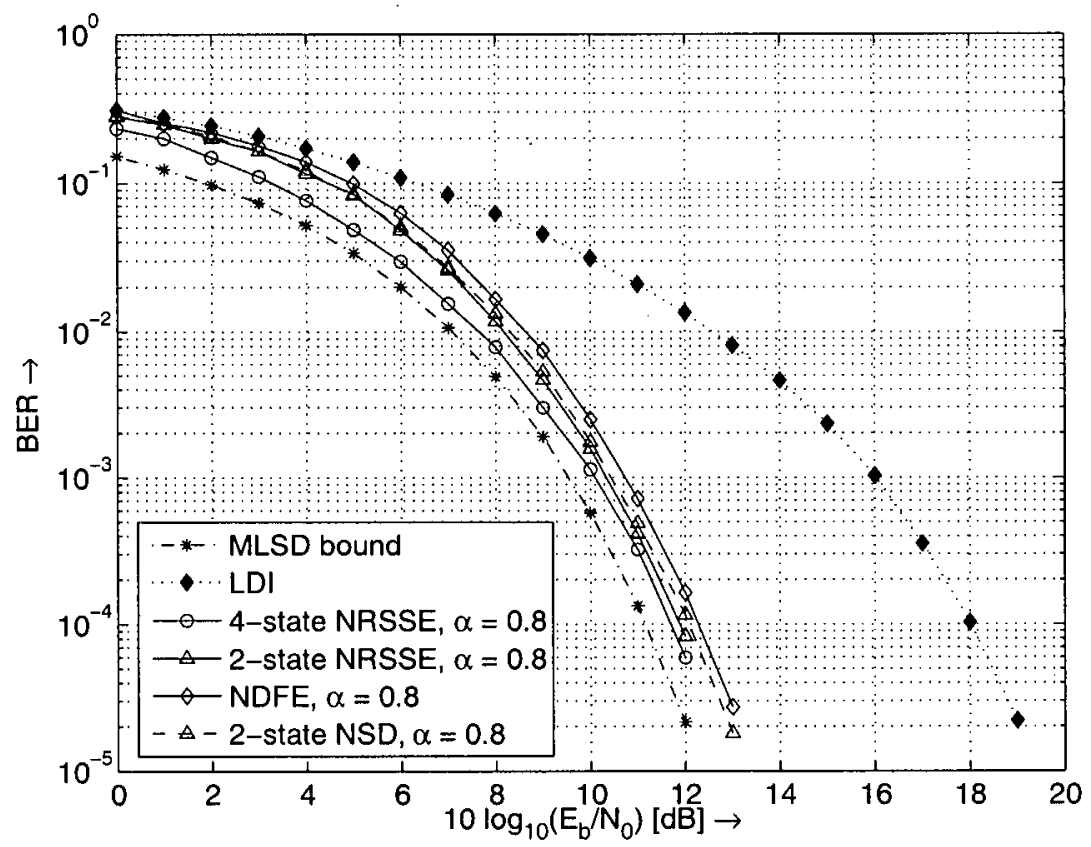

Figure 4.4: Evaluation of the performance of the proposed receiver with state reduction in case of noncoherent detection.

\subsubsection{Performance with Constant Channel Phase}

In this section, the channel phase is assumed to be time-invariant. The performance under this condition is evaluated with state reduction, and for different values of $N$ and $\alpha$. Moreover, we discuss the effect of the varying modulation index at the transmitter compared to the results obtained when $h$ is known at the receiver. We remind the reader that the results presented in this work assume the FBF and FFF filters are fixed according to $h=1 / 3$. Only negligible, if any, performance loss incurs compared to the case where $h$ is varied according to the actual value at the transmitter, as illustrated in Fig. 4.3.

\section{State Reduction}

We previously found that the DFE suffers fairly acceptable performance losses with respect to the 2-state and 4-state RSSE. In this section, we illustrate that a similar result is obtained in case of noncoherent detection and compare the performance of the 
proposed receiver to the LDI and the NSD. Fig. 4.4 shows that NRSSE and NSD yield similar performance. In fact, the BER curve of the 2-state NRSSE almost coincides with that of the 2-state NSD. Moreover, we notice that NDFE suffers a performance loss of about $0.3 \mathrm{~dB}$ and $0.4 \mathrm{~dB}$ compared to 2-state NRSSE and 2-state NSD, respectively, and achieves a performance gain of about $5.5 \mathrm{~dB}$ over the LDI. As further state reduction turned out impossible for the NSD approach of [27] based on Rimoldi decomposition, we can deduce that Laurent's decomposition is preferable for the design of low-complexity Bluetooth receivers. Finally, we conclude that NDFE provides an excellent tradeoff between performance and complexity, making it the ideal equalization scheme for the proposed receiver. Hence, the following discussion will focus on the performance evaluation of NDFE.

\section{Performance with Different Values of $N$ and $\alpha$}

Starting with the $N$-metric (3.45), the BER curves for NDFE with different values of $N$ are shown in Fig. 4.5. As expected, without channel phase variations, as the value of $N$ increases, the performance closely approaches that of coherent DFE. We also observe that even for the minimum value of $N=2$, we have a performance improvement of more than $2.5 \mathrm{~dB}$ over the LDI receiver.

Fig. 4.6 shows the performance using the $\alpha$-metric. With $\alpha=0.95$, the performance difference between coherent DFE and NDFE is a negligible $0.06 \mathrm{~dB}$. Similar to $N$, as $\alpha$ increases, we get better power efficiency.

Moreover, recalling Eq. (3.47), which compares the performance of the NDFE using the $N$ - and $\alpha$-metric, it may be verified by a careful observation of Figs. 4.5-4.6. Specifically, we see that the performance for $N=10$ is equivalent to that of $\alpha=0.8$, and a similar statement is true for $N=2,5$ and $\alpha=0,0.6$, respectively. However, since the $\alpha$-metric is computationally less complex (cf. Section 3.6), the following discussion will be solely based on the $\alpha$-metric. 


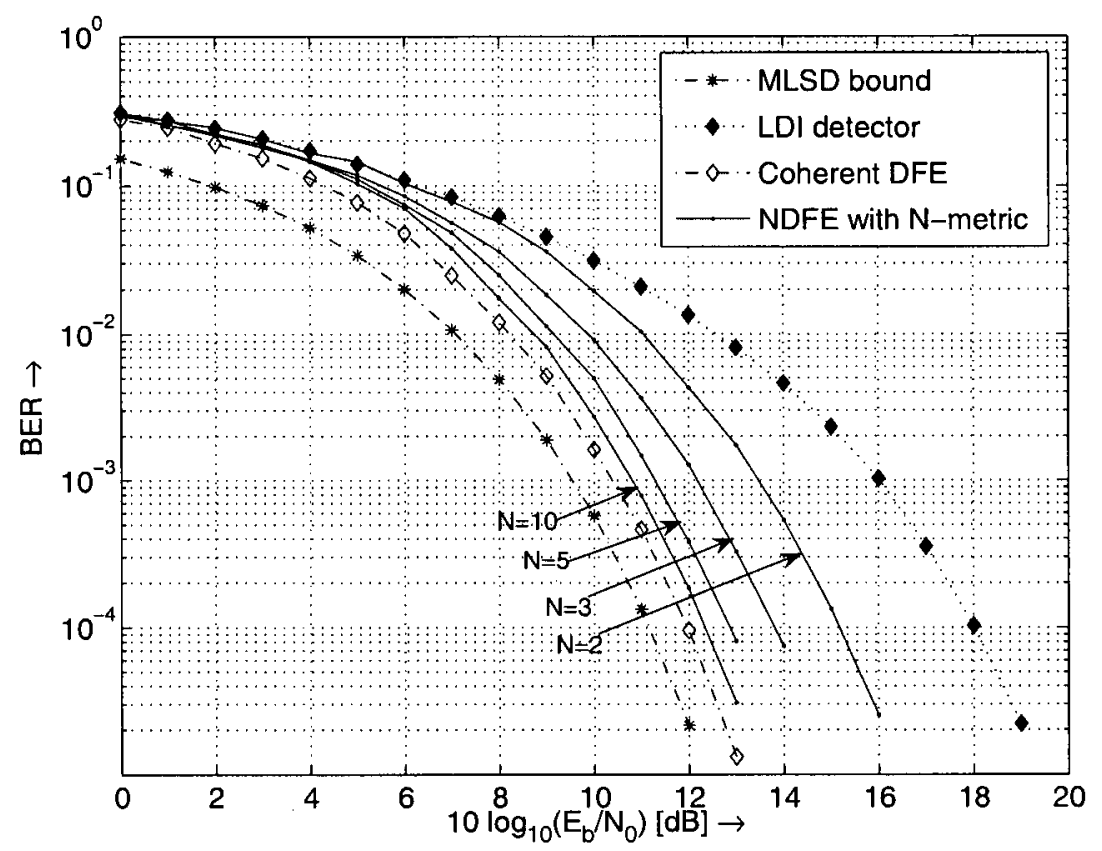

Figure 4.5: Performance of the NDFE using the $N$-metric compared to MLSD, LDI, and coherent DFE, and $h=1 / 3$ for all cases.

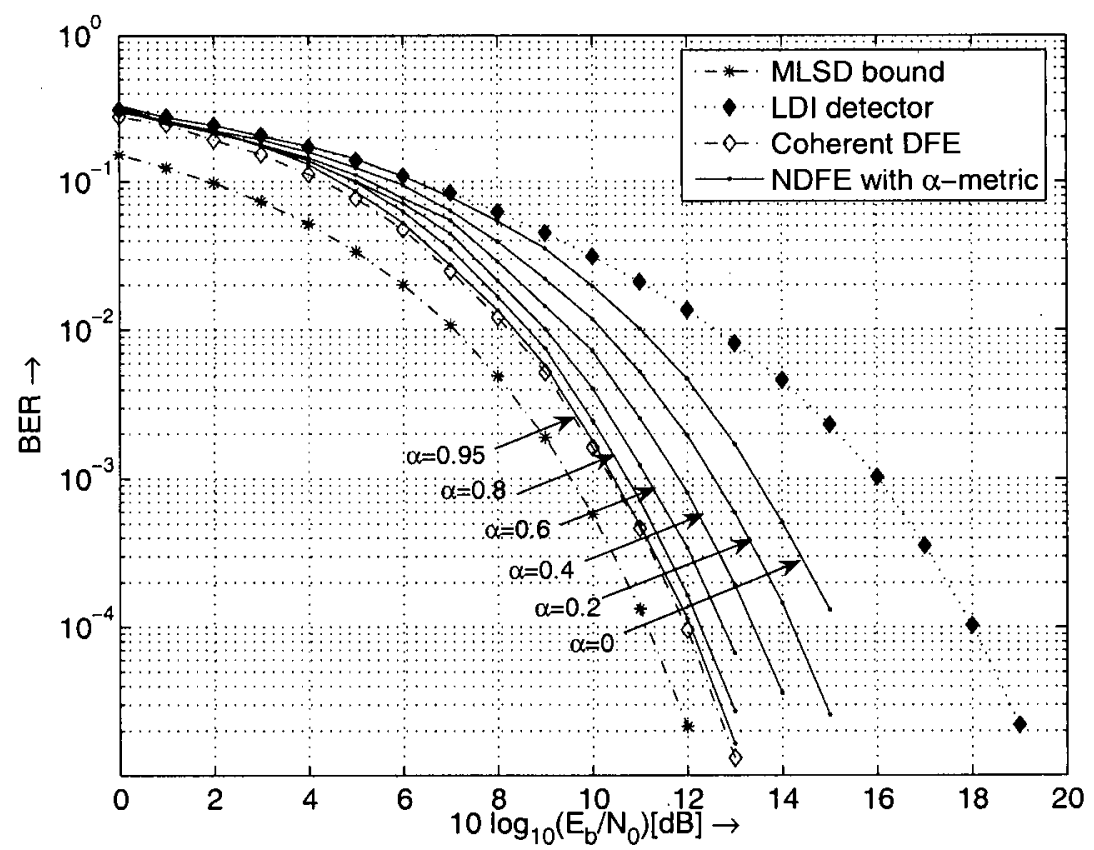

Figure 4.6: Performance of the NDFE using the $\alpha$-metric compared to MLSD, LDI, and coherent DFE, and $h=1 / 3$ for all cases. 


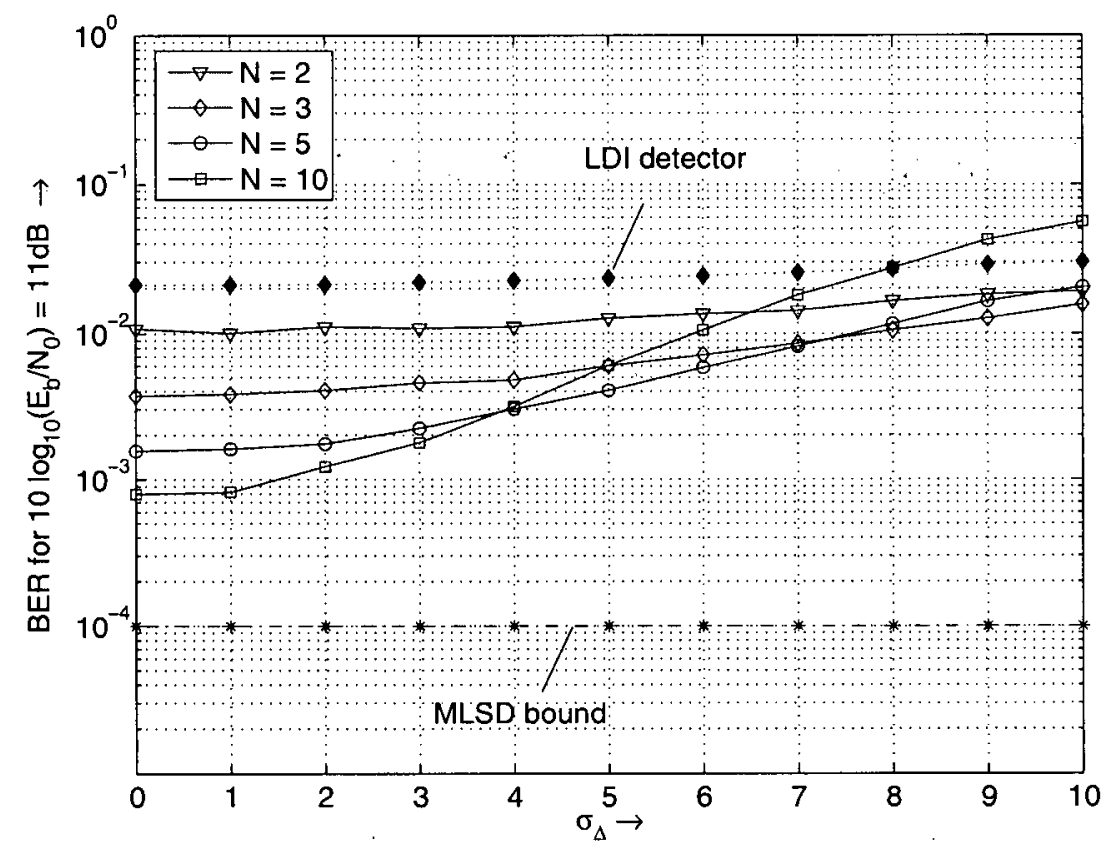

Figure 4.7: Performance of the NDFE using the $N$-metric $h=1 / 3$ in the presence of phase jitter.

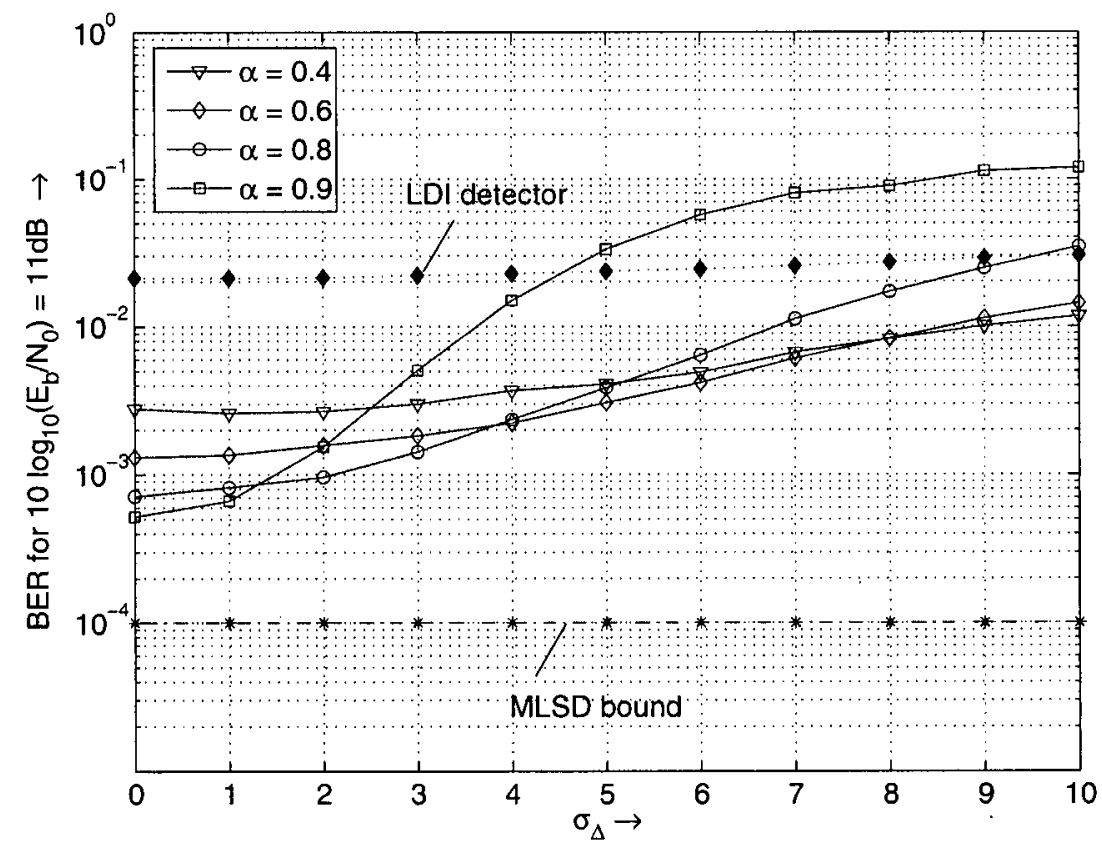

Figure 4.8: Performance of NDFE using the $\alpha$-metric $h=1 / 3$ in the presence of phase jitter. 
As mentioned above, increasing the value of $\alpha$ and $N$ achieves better performance when the channel phase is constant. However, this is not the case in the presence of phase jitter, as can be seen in Fig. 4.7 which plots the BER with respect to the standard deviation of the phase jitter $\sigma_{\Delta}$. In this case, the performance of the proposed receiver deteriorates as $N$ increases since the assumption of constant channel phase is no longer valid. A similar observation can be made when using the $\alpha$-metric, as shown in Fig. 4.8. Therefore, the optimal value of $N$ or $\alpha$ depends on the channel conditions, and we may conclude that NDFE with $N$ between 5 and 10 or $\alpha$ between 0.6 and 0.8 is power efficient and gives satisfactory performance in the presence of phase jitter.

\section{Performance with Different Modulation Indices}

In the previous sections, we have assumed $h=1 / 3$ at the transmitter and receiver. However, since the GFSK signal depends on $h$ [Eqs. (2.1)-(2.2)], even with the assumption that $h$ is known at the receiver, different performance results are obtained for different modulation indices. Specifically, the performance deteriorates as $h$ decreases since $f_{d}$ (cf. Section 2.1.4) decreases. Fig. 4.9 shows the performance of NDFE with $\alpha=0.8$ for $h=0.28,1 / 3$, and 0.35 , along with the corresponding MLSD bounds, the LDI, and the MLM-LDI ${ }^{1}$. The performance of NDFE, which is equivalent to a one-state trellis gives remarkable results when compared to the MLSD bound with a performance loss of only about $1.2 \mathrm{~dB}$. Recall that the optimum receiver (cf. Section 2.2.1) requires 100 trellis states for $h=0.28$ and 80 trellis states for $h=0.35$, while the optimal receiver based on Laurent's decomposition (cf. Section 3.1.2) requires 50 and 40 states for $h=0.28$ and $h=0.35$, respectively. The performance gains compared to the LDI and MLM-LDI are more than $5 \mathrm{~dB}$ and $1 \mathrm{~dB}$, respectively.

\footnotetext{
${ }^{1}$ The results for the MLM-LDI are taken from [49].
} 


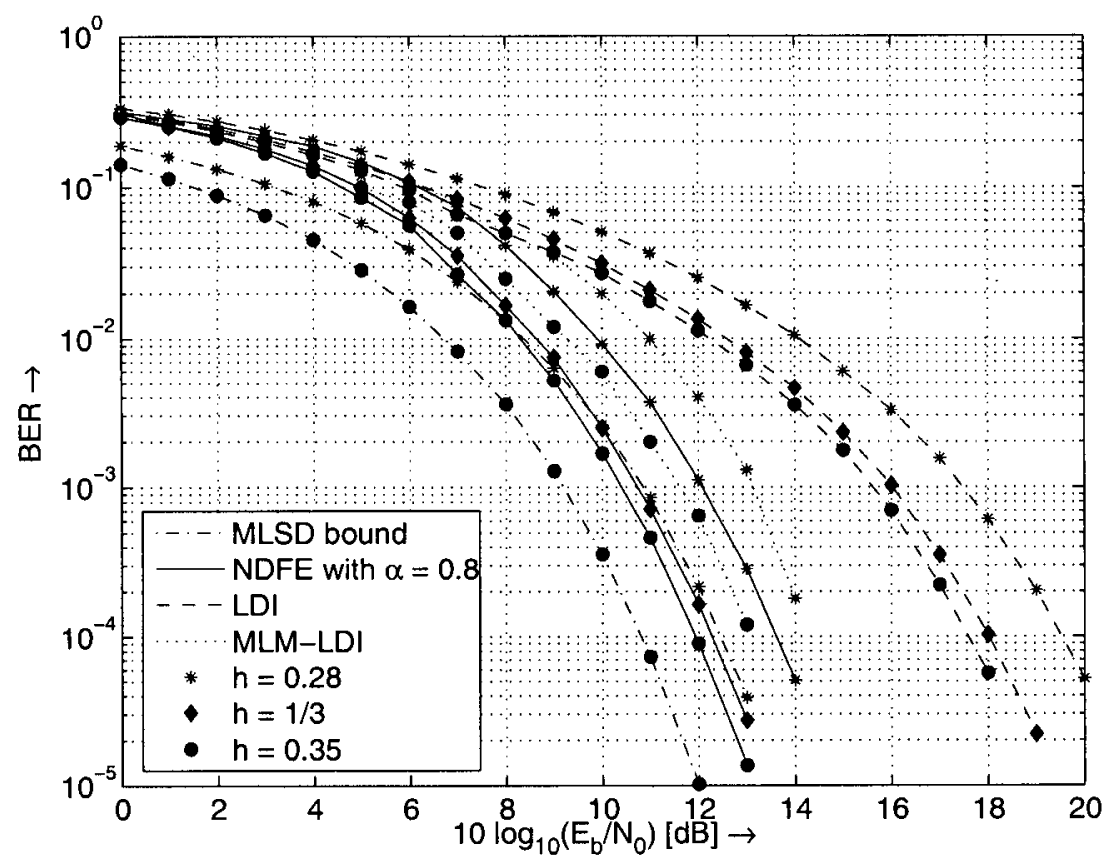

Figure 4.9: Performance of the NDFE with $\alpha=0.8$ for different values of the modulation index compared to the MLSD bound, LDI, and MLM-LDI.

\section{Performance with Unknown Modulation Index}

So far, we have assumed the modulation index $h$ to be known at the receiver. In Bluetooth devices, the varying modulation index $(0.28 \leq h \leq 0.35)$ poses a serious challenge for the receiver design. To support this statement, we show the BER obtained for an SNR of $11 \mathrm{~dB}$ for different values of $\alpha=0.4,0.6,0.8$, and 0.9 when a fixed modulation index $h=0.28,0.30,1 / 3$, and 0.35 is assumed at the receiver. The simulations are performed for different values of the actual modulation index of the transmitted signal, and the obtained results are shown in Fig. 4.10. It is clear that as $h$ at the receiver deviates from the actual $h$ of the transmitted signal, the performance degrades. We also notice that the performance loss with the $h$ deviation is more severe for greater values of $\alpha$. This is due to the fact that a mismatch of $h$ at the receiver takes longer to forget in case of a greater forgetting factor $\alpha$. Hence, a lower value of $\alpha$ provides more robustness against variations in the modulation index. Consider, for example, $\alpha=0.4$, a nominal value of $h$ at the receiver would result in very slight changes from the case 

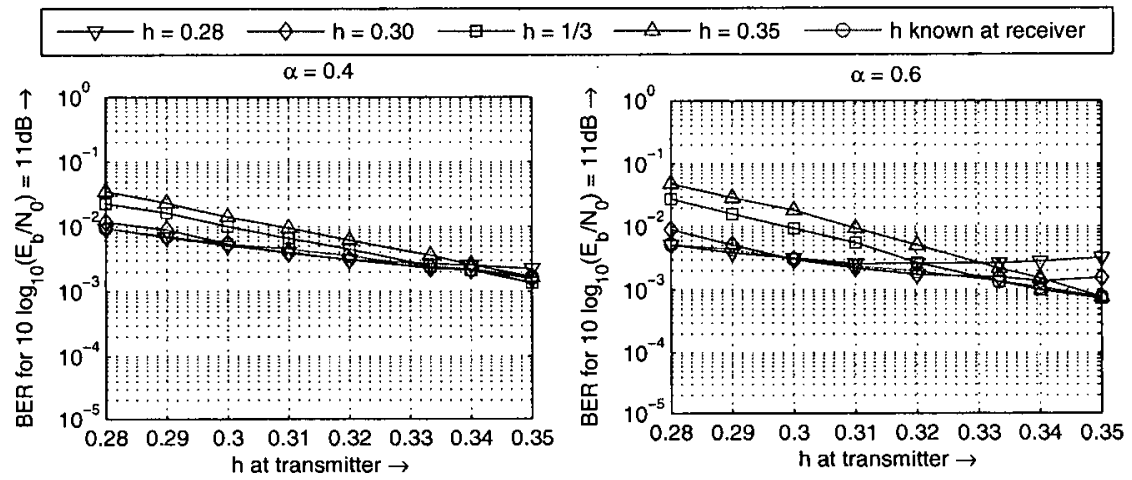

$\alpha=0.8$

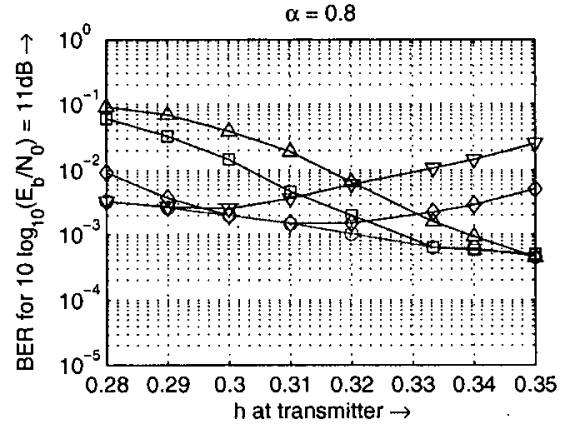

$\alpha=0.9$

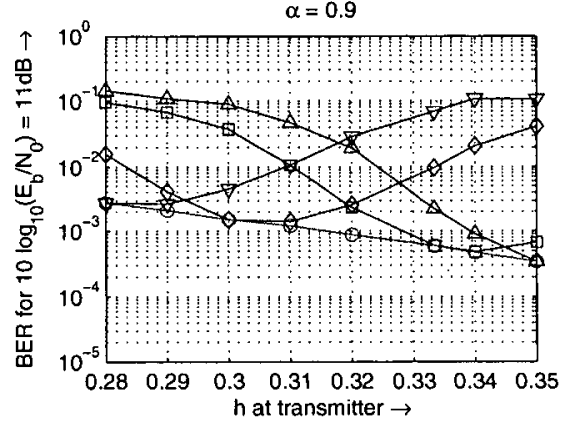

Figure 4.10: Performance of the NDFE for varying $h$ with $\alpha=0.4,0.6,0.8$, and 0.9 .
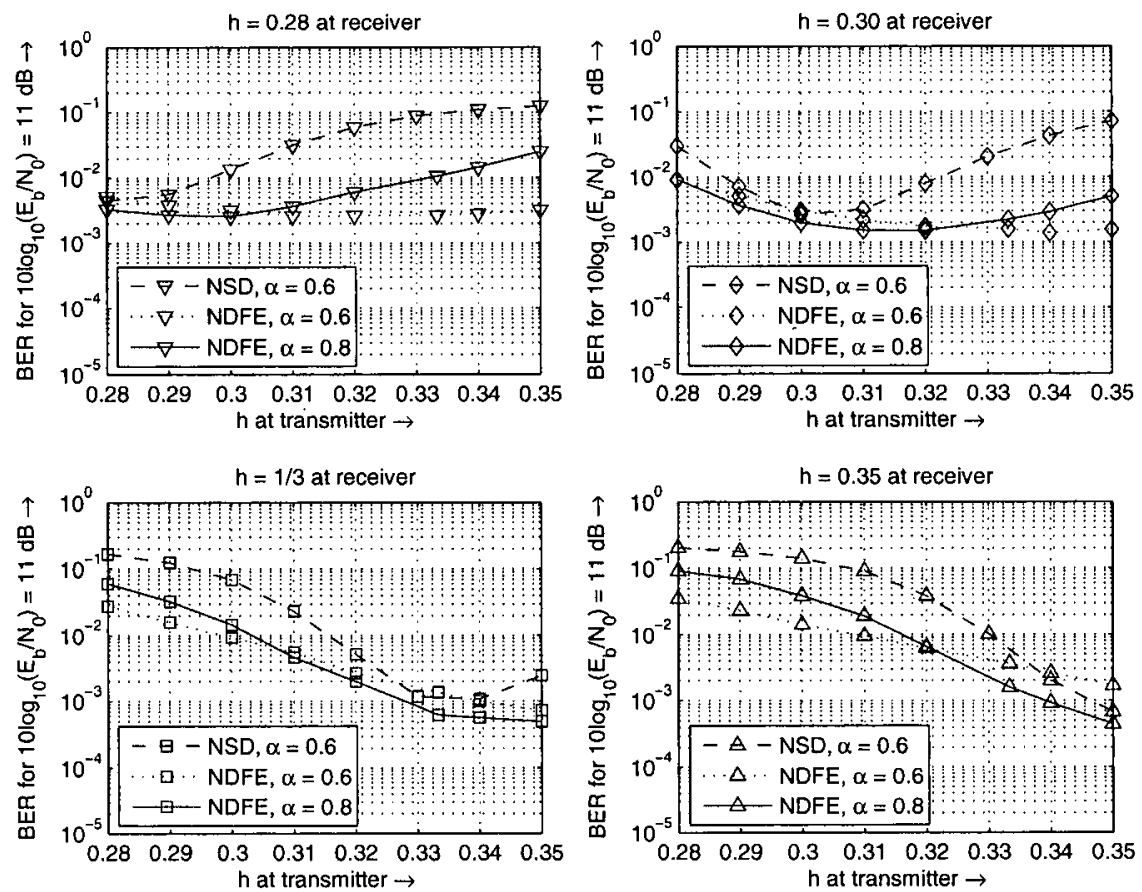

Figure 4.11: Performance of NDFE for varying $h$ with $\alpha=0.6$ and 0.8 compared to that of NSD for $\alpha=0.6$. 
when $h$ is known at the receiver but still achieve more power efficiency than the LDI.

To evaluate the proposed receiver's robustness against $h$ variations with respect to the alternative noncoherent sequence detector in literature, namely, NSD, we show a relative comparison in Fig. 4.11. The BER curves obtained for SNR $=11 \mathrm{~dB}$ are shown for various modulation indices $h=0.28,0.3,1 / 3$, and 0.35 at the receiver, and are plotted for a varying $h$ at the transmitter. We choose the curves for NSD with $\alpha=0.6$ since it was found to be the optimal value of $\alpha$ for NSD [27]. The NSD curves are compared to of NDFE with $\alpha=0.6$ and 0.8 . The presented results show that as $h$ varies, a much greater deviation is observed in the BER curves for NSD compared to those of NDFE, both with $\alpha=0.6$. Considering the case when $h=0.28$ at the receiver, as $h$ varies at the transmitter, the corresponding BER is almost constant in case of NDFE, which is not the case for NSD. Specifically, the resulting percentage of error varies between $0.25 \%$ and $0.5 \%$ in NDFE, and between $0.4 \%$ and $12 \%$ in NSD. Moreover, we even observe less change in the BER of NDFE for $\alpha=0.8$ compared to NDFE with $\alpha=0.6$. We conclude that NDFE is more robust against $h$ variations compared to NSD.

A more representative evaluation of the effect of varying modulation index is obtained by considering the required SNR for a BER of $10^{-3}$. We adopt the same scenario as before. Fig. 4.12 shows the required SNR for a BER of $10^{-3}$ as a function of the modulation index $h$ for NDFE with $\alpha=0.8$ and for the MLSD bound and the LDI for comparison purposes. The figure also illustrates the ideal case of $h$ known at the receiver. As can be observed, severe power efficiency losses result when $h$ is unknown at the receiver.

The variations in the modulation index may be minimized by estimating $h$ at the transmitter using ANDFE described in Section 3.6.3, and the corresponding results will be presented later in this chapter. 


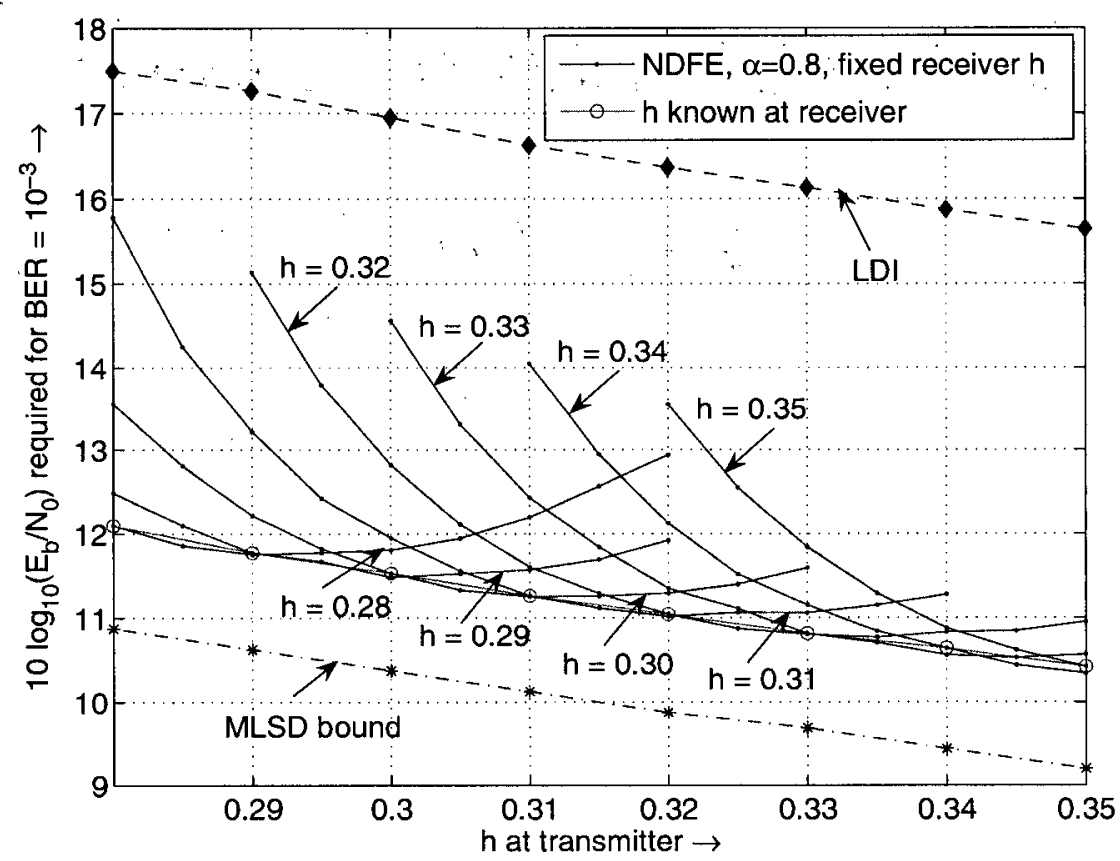

Figure 4.12: The required $10 \log _{10}\left(E_{b} / N_{0}\right)$ for $\mathrm{BER}=10^{-3}$ for NDFE with $\alpha=0.8$ with varying modulation index.

\subsubsection{Performance Using the Modified Phase Reference Un- der Constant and Varying Channel Conditions}

The more realistic and thus more relevant transmission scenario considers a timevarying channel phase $\phi(t)$. In the present work, we account for channel phase variations through the modified phase reference [see Eqs. (3.53)-(3.54)] formulated in terms of two forgetting factors $\alpha$ and $\beta$. Throughout this chapter, if $q_{\mathrm{ref}}[k]$ from (3.46) is used, only the value of $\alpha$ is specified, and if $q_{\mathrm{ref}}[k]$ from (3.53) is applied, the values of both $\alpha$ and $\beta$ are given.

\section{Performance With Constant Channel Phase}

Before considering a time-variant channel phase, we first show the performance of the modified phase reference when the channel phase is constant. Fig. 4.13 illustrates the performance for different values of $\alpha$ and $\beta$ as a function of the modulation index, 


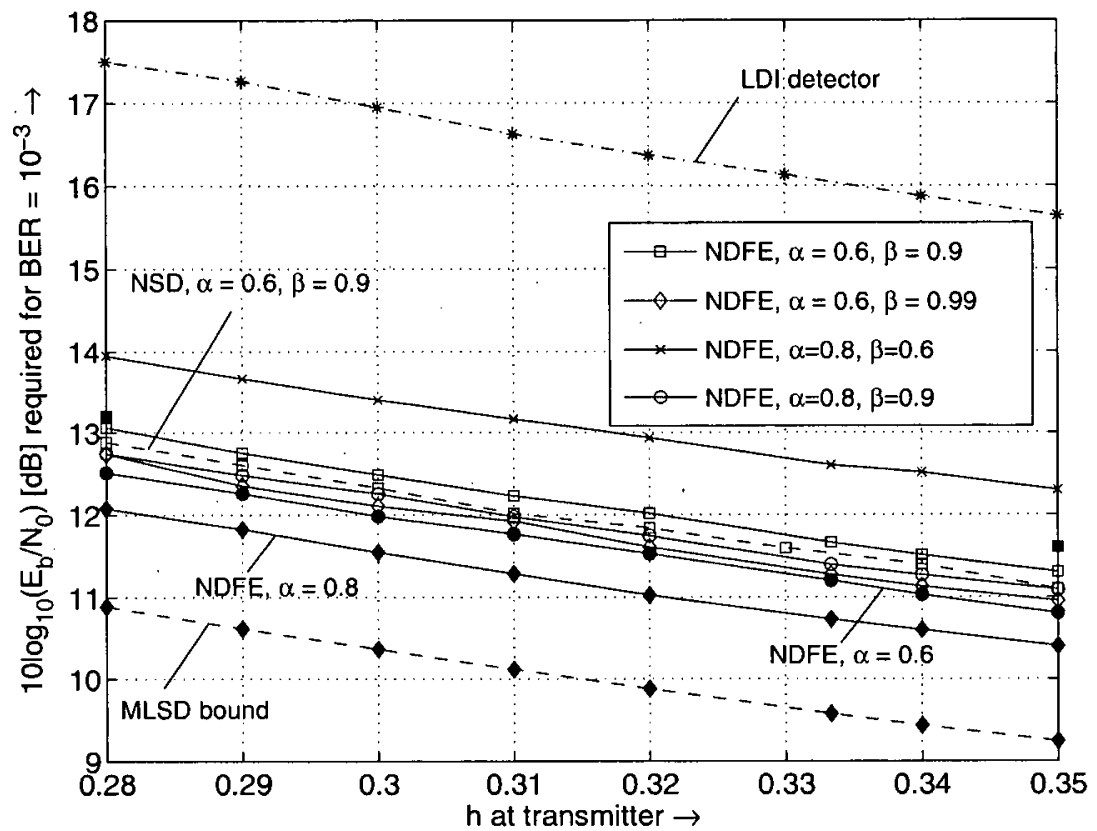

Figure 4.13: $10 \log _{10}\left(E_{b} / N_{0}\right)$ required for BER $=10^{-3}$ for different combinations of $\alpha$ and $\beta$ as a function of the modulation index with constant channel phase and $h$ known.

which is assumed to be known at the receiver. We notice that there is a loss in power efficiency when (3.53) is used compared to when (3.46) is applied due to the phase noise introduced by the frequency estimation. More specifically, the gap between $\alpha=0.6$ and the pair $(\alpha=0.6, \beta=0.9)$ is approximately $0.4 \mathrm{~dB}$. This loss for the ideal case of a constant channel phase has to be accepted to achieve a high performance in the more realistic scenario of a time-varying phase. Moreover, we observe that as $\beta$ increases the performance of $q_{\mathrm{ref}}[k]$ from (3.53) closely approaches that of $q_{\mathrm{ref}}[k]$ from (3.46). It was found that for various scenarios, the pair $(\alpha=0.6, \beta=0.9)$ appeared to be a favorable choice. We further observe that the robust NDFE with $(\alpha=0.6, \beta=0.9)$ outperforms the LDI detector by more than $4 \mathrm{~dB}$, and it is still somewhat more power efficient than the MLM-LDI detector, which requires a four-state forward-backward algorithm. Finally, we note that NDFE with frequency-offset estimation performs very similar to the more complex NSD with the same values of $\alpha$ and $\beta$. 


\section{Performance with Varying Modulation Index}

As for the effect of the $\beta$ factor on the varying modulation index, the resulting performances of NDFE with $(\alpha=0.6, \beta=0.9)$ and $(\alpha=0.8, \beta=0.9)$ are shown in Figs. 4.14 and 4.15 , respectively. The curves represent the required SNR for BER = $10^{-3}$ for fixed values of the modulation index at the receiver as function of a varying modulation index at the transmitter. We notice that the performance degradation resulting from a mismatch in $h$ encountered using (3.46) persists when using (3.53). As expected, when $\alpha$ decreases the SNR differences between unknown $h$ at the receiver and known $h$ decreases. Regardless, using the modulation index estimator (cf. Section 3.6.3) is advisable, and will be presented in the Section 4.2.3.

\section{Performance With Varying Channel Phase}

The modified phase reference (3.53) was developed to account for the extreme (yet admissible) phase variations and frequency offsets of $\Delta f T=0.1$ in Bluetooth devices. We now evaluate the performance of NDFE using this modified phase reference in the presence of frequency offset and phase jitter. Fig. 4.16 shows the required SNR for $\mathrm{BER}=10^{-3}$ as a function of the normalized frequency offset. The curves are parameterized with a standard deviation of $\sigma_{\Delta}=0^{\circ}$ (no jitter) and $\sigma_{\Delta}=2^{\circ}, 5^{\circ}$ for the phase jitter. The LDI detector fails for offsets $\Delta f T>0.03 \ldots 0.05$, but we note that DC offset cancellation methods could be applied to mitigate the effect of frequency offset. We further observe that NDFE with phase reference (3.46) cannot cope with large frequency offsets of $\Delta f T \gtrsim 0.02$. However, considering the pair $(\alpha=0.6, \beta=0.9)$,

the NDFE with phase reference (3.53) allows power-efficient transmission even for extreme offsets: In addition, the maximum performance degradation when assuming an additional phase jitter with $\sigma_{\Delta}=5^{\circ}$ is not more than $1.5 \mathrm{~dB}$.

For the same scenario, Fig. 4.17 compares the performances of NDFE for the two pairs $(\alpha=0.6, \beta=0.9)$ and $(\alpha=0.8, \beta=0.9)$ and $\mathrm{NSD}$ with the pair $(\alpha=0.6, \beta=0.9)$, 


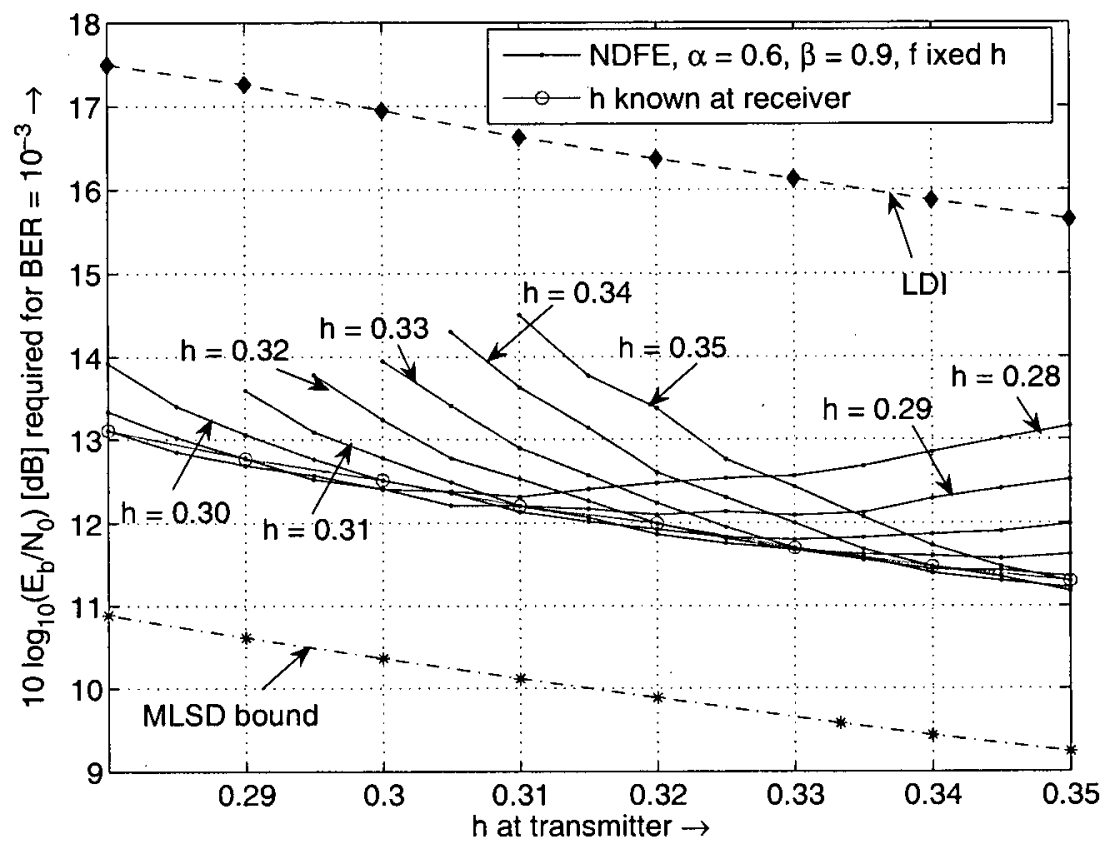

Figure 4.14: The required $10 \log _{10}\left(E_{b} / N_{0}\right)$ for BER $=10^{-3}$ for NDFE with $\alpha=0.6, \beta=$ 0.9 for varying $h$.

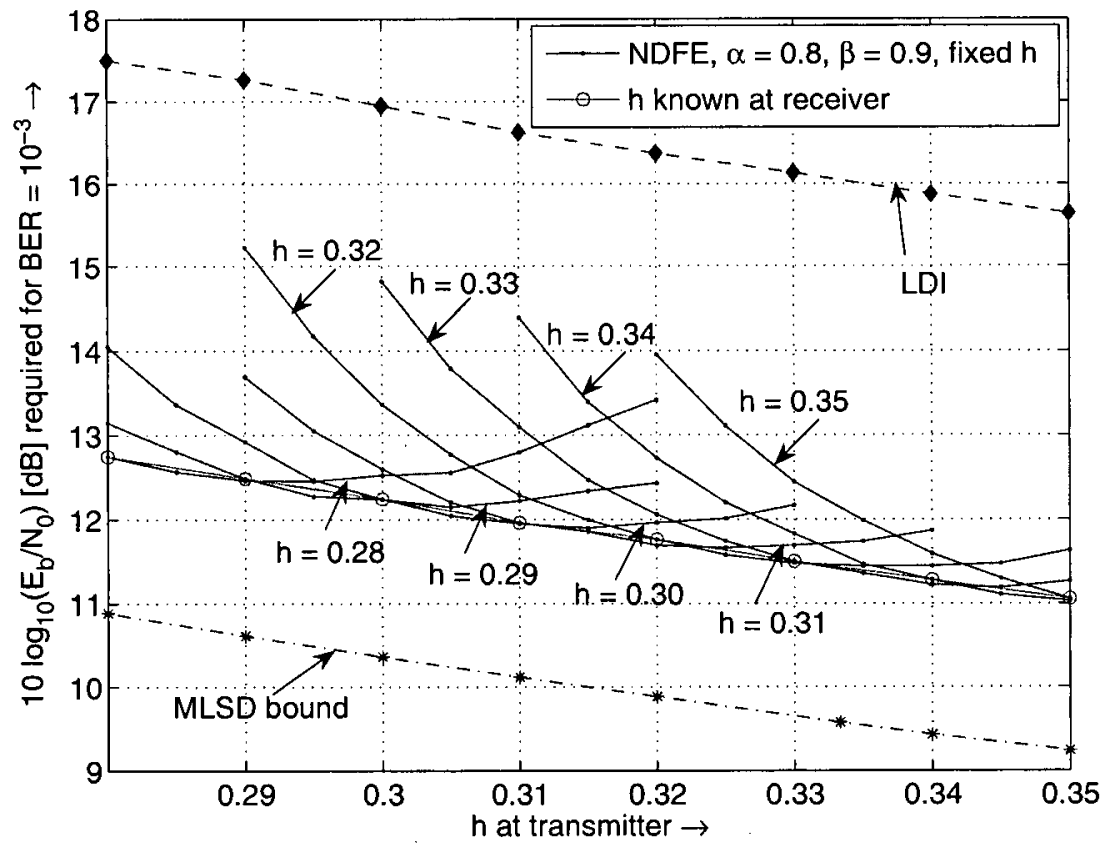

Figure 4.15: The required $10 \log _{10}\left(E_{b} / N_{0}\right)$ for $\mathrm{BER}=10^{-3}$ for NDFE with $\alpha=0.8, \beta=$ 0.9 for varying $h$. 


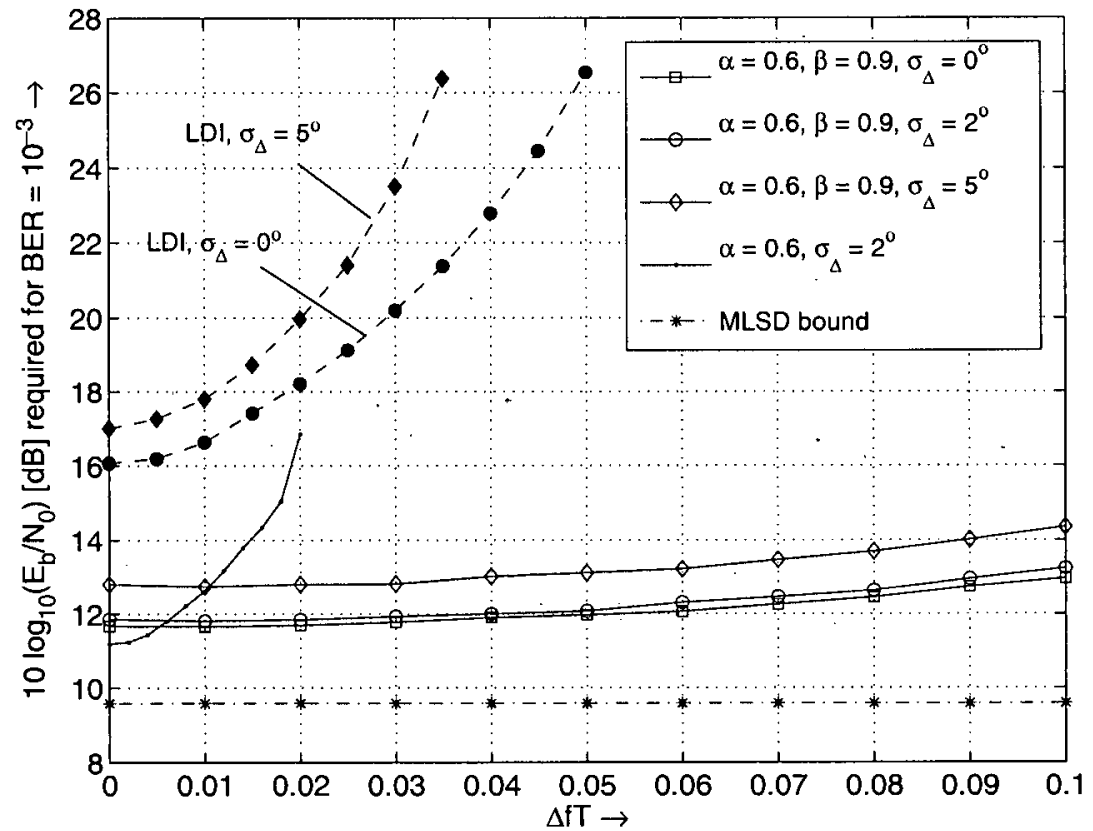

Figure 4.16: The required $10 \log _{10}\left(E_{b} / N_{0}\right)$ for $\mathrm{BER}=10^{-3}$ in the presence of frequency offset and phase jitter.

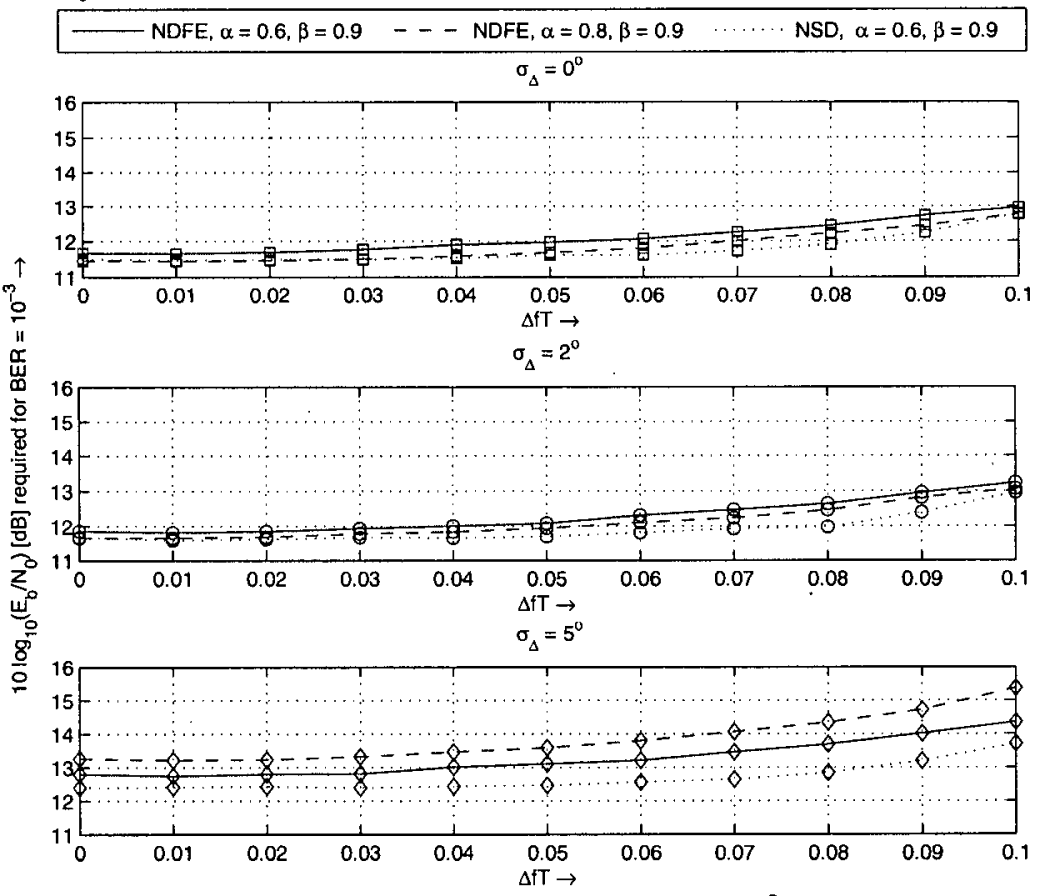

Figure 4.17: The required $10 \log _{10}\left(E_{b} / N_{0}\right)$ for BER $=10^{-3}$ in the presence of frequency offset and phase jitter. 
which was found most favorable in [27]. We observe that the performance deteriorates with increasing phase jitter as $\alpha$ increases (both with $\beta=0.9$ ). The NDFE with the pair ( $\alpha=0.6, \beta=0.9$ ) was found to have comparable performance with the 2 -state NSD. The preferable performance for $(\alpha=0.6, \beta=0.9)$ in the presence of extreme phase variations is one of the reasons why this pair is considered to be the favorable choice for NDFE.

\subsubsection{Adaptive NDFE}

The performance of NDFE severely deteriorates when there is a mismatch between the value of $h$ assumed at the receiver and the actual $h$ at the transmitter, as was illustrated in the previous sections (cf. Figs. 4.12, 4.14, 4.15). We now evaluate the performance of ANDFE (cf. Section 3.6.3), where several NDFEs are executed for a number of hypothetical modulation indices, and the decision metrics are accumulated. We consider different combinations of an estimation period $N_{e}=25$ or 50 , and a set of 2 or 4 hypothesis $\mathcal{H}$. When $|\mathcal{H}|=2$, the hypothetical modulation index set $\mathcal{H}=\{0.3,1 / 3\}$, while $\mathcal{H}=\{0.28,0.30,0.32,0.34\}$ with $|\mathcal{H}|=4$. In the following, we adopt the notation $\operatorname{ANDFE}\left(N_{e},|\mathcal{H}|\right)$ to convey the estimation period and hypothetical modulation set used.

Fig. 4.18 plots the SNR required for $\mathrm{BER}=10^{-3}$ vs. the modulation index used at the transmitter for NDFE with $h$ known at the receiver and ANDFE with $h$ unknown at the receiver. The phase reference (3.46), which does not account for frequency offset variations, is used for each of the values $\alpha=0.6$ and $\alpha=0.8$. Observing the results obtained for $\alpha=0.8$, we notice that the curves for $\operatorname{NDFE}$ and $\operatorname{ANDFE}(50,4)$ are very close, and the ANDFE shows a remarkable performance improvement compared to the case where $h$ is unknown at the receiver and equal to 0.32 , reaching $3 \mathrm{~dB}$ when $h$ at the transmitter is 0.29 . For $\alpha=0.6$ it is illustrated that even for a small estimation period and modulation index hypothesis of $N_{e}=25$ and $|\mathcal{H}|=2$, the ANDFE is still 


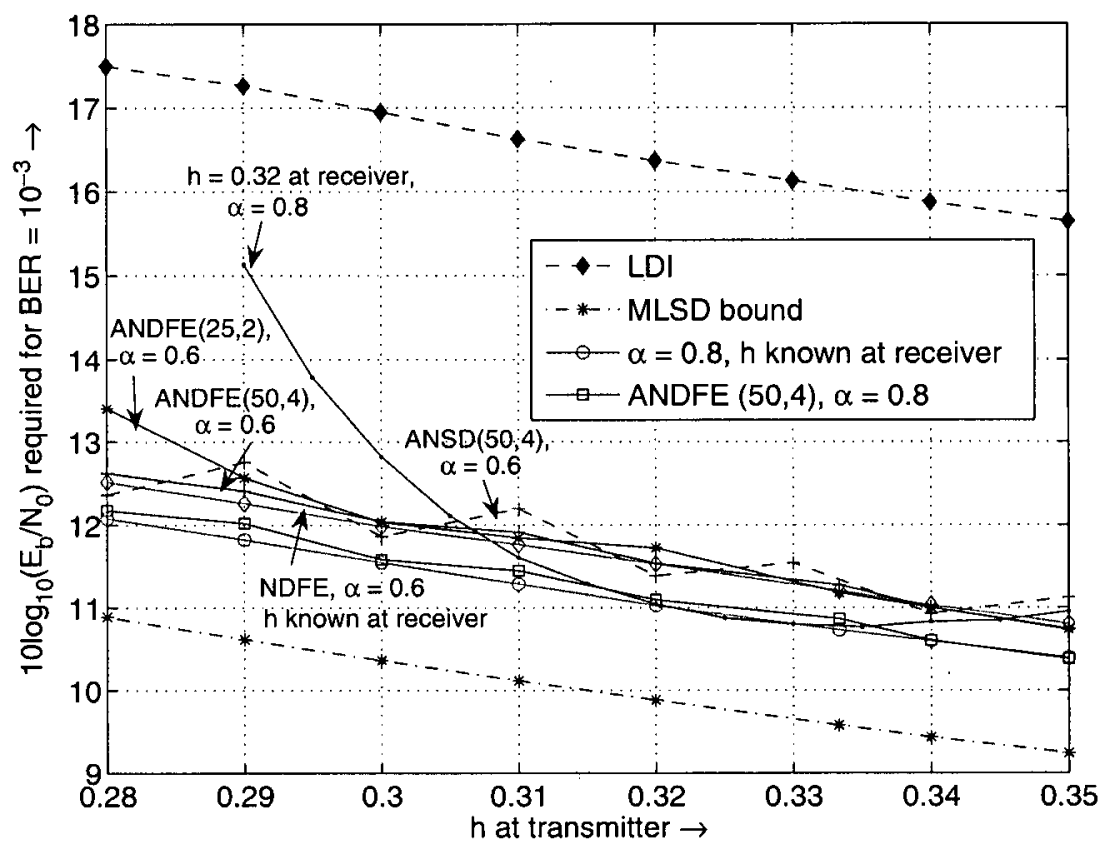

Figure 4.18: $10 \log _{10}\left(E_{b} / N_{0}\right)$ required for $\mathrm{BER}=10^{-3}$ for ANDFE with phase reference (3.46). The channel phase is constant and the notation $\operatorname{ANDFE}\left(N_{e}, \mathcal{H}\right)$ applies.

robust. Again, the results for ANDFE and adaptive NSD (ANSD) ${ }^{2}$ with $\alpha=0.6$ are almost identical, as has been observed for the various aforementioned scenarios.

In Fig. 4.19, we illustrate the performance of ANDFE for different pairs of $\alpha$ and $\beta$ when the phase reference (3.53) with frequency offset estimation is used. For comparison, the results of $\operatorname{ANDFE}(50,4)$ with phase reference (3.46) and $\alpha=0.6$ are also shown. We make the following observations. First, it is evident that the adaptive version of NDFE (ANDFE) with an estimation period $N_{e}=50$ combined with $|\mathcal{H}|=4$ and $h$ unknown at the receiver achieves a result almost identical to NDFE with $h$ known, as illustrated for the pair $(\alpha=0.6, \beta=0.8$ ). Second, the results obtained for almost all combinations of $\alpha$ and $\beta$ form almost straight lines, indicating that they are close to the $h$ known case. Third, as $\beta$ approaches 1 , the performance of ANDFE using the phase reference (3.53) approaches that of (3.46), which is consistent with the results

\footnotetext{
${ }^{2}$ The ANSD is the NSD receiver with an adaptive $h$-estimator.
} 


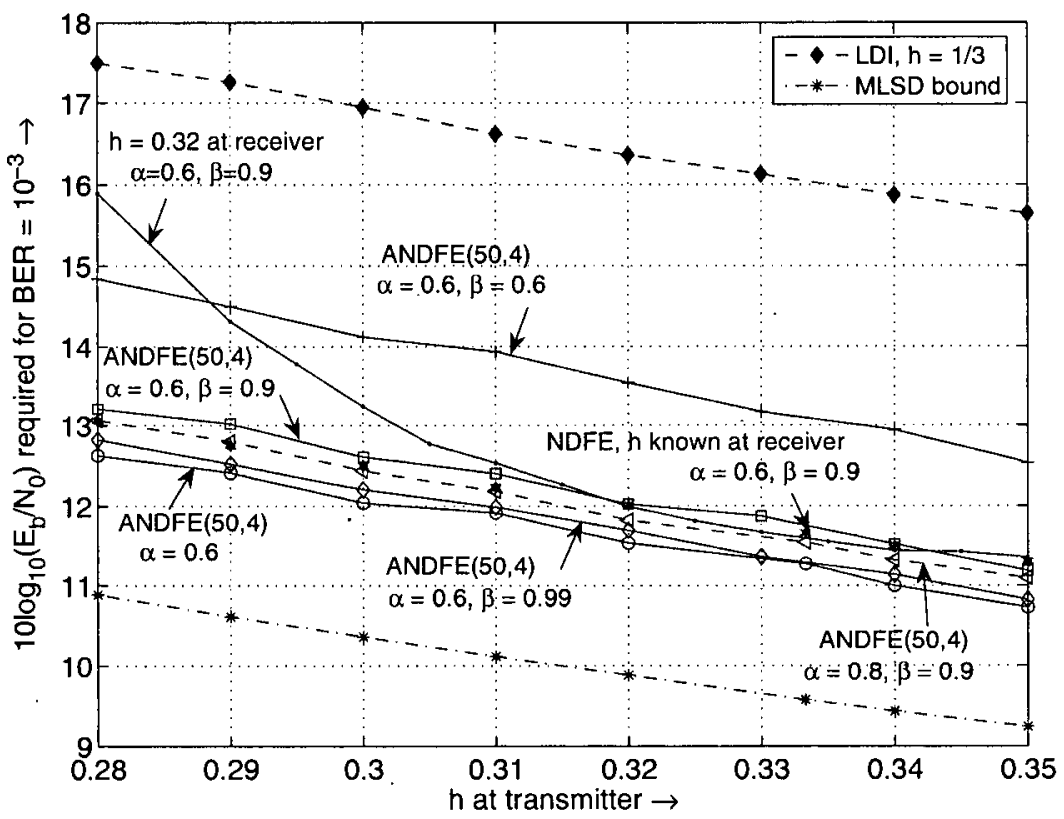

Figure 4.19: $10 \log _{10}\left(E_{b} / N_{0}\right)$ required for $\mathrm{BER}=10^{-3}$ for $\mathrm{ANDFE}$ with various combinations of $\alpha$ and $\beta$ with phase reference (3.53). The channel phase is constant and the notation $\operatorname{ANDFE}\left(N_{e}, \mathcal{H}\right)$ applies.

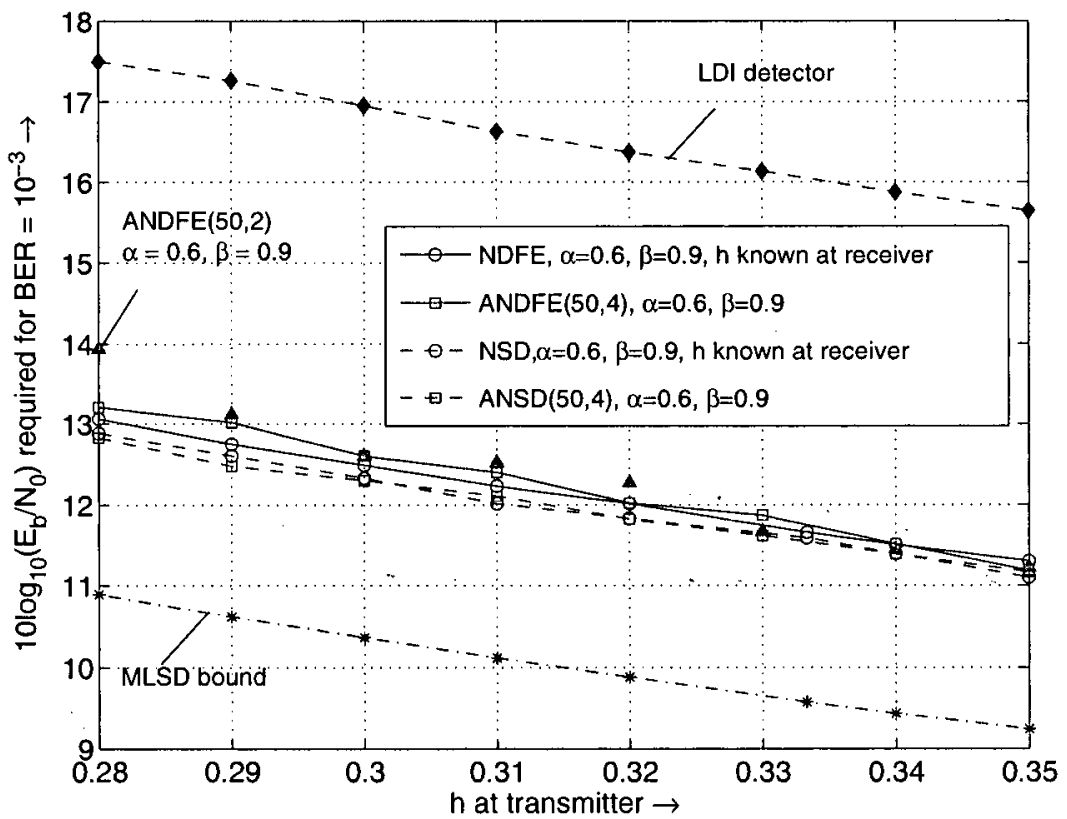

Figure 4.20: $10 \log _{10}\left(E_{b} / N_{0}\right)$ required for BER $=10^{-3}$ for ANDFE and ANSD with the favorable pair $(\alpha=0.6, \beta=0.9)$ and with constant channel phase. 
previously shown for NDFE.

Fig. 4.20 compares the performance of the ANDFE with the adaptive NSD (ANSD), which employs an adaptive scheme similar to that of ANDFE. The results show very close performance between the adaptive schemes of NDFE and NSD when the same values of $\alpha$ and $\beta$ are used (the maximum difference is about $0.4 \mathrm{~dB}$ ), even with a smaller set of $|\mathcal{H}|=2$. We can thus conclude that NDFE allows highly power-efficient detection also for a priori unknown modulation index.

\subsubsection{Performance in the Presence of Interference}

Due to the operation of Bluetooth in the ISM band, evaluating the proposed Bluetooth receiver in the presence of interference is necessary. We consider the performance with interference, such that the signal-to-interference power ratio (SIR) and the carrier frequency difference $\Delta f_{c, i}$ are chosen as specified in the Bluetooth standard and presented in Table 2.1. The notation $\operatorname{IF}\left(\operatorname{SIR}, \Delta f_{c, i}\right)$ is used to convey the SIR in $\mathrm{dB}$ and $\Delta f_{c, i}$ in MHz. Fig. 4.21 shows the performance results for the optimal WMF and the proposed SRC filter, where $h=1 / 3$ is known at the receiver. The effective interference suppression provided by both filters is verified, with the BER for adjacent channel interference almost equal to the curve obtained when no interference is considered. The results for both filters are quite similar, with the SRC outperforming the WMF in case of adjacent channel interference, and the reverse is observed with co-channel interference. As a matter of fact, when the SRC filter is applied, there is no loss due to adjacent channel interference for the cases $\operatorname{IF}(-30,2)$, and $\operatorname{IF}(-40,3)$. Moreover, the BER curve for $\operatorname{IF}(-$ $40,3)$ is shown when the SRC is applied without the Hamming window, and a loss of $\simeq 0.5 \mathrm{~dB}$ is observed compared to the case when the Hamming window is applied.

Fig. 4.22 illustrates the performance of the ANDFE in the presence of interference as a function of the modulation index. We observe that the results of ANDFE are almost identical to those for NDFE in the presence of adjacent channel interference. As for 


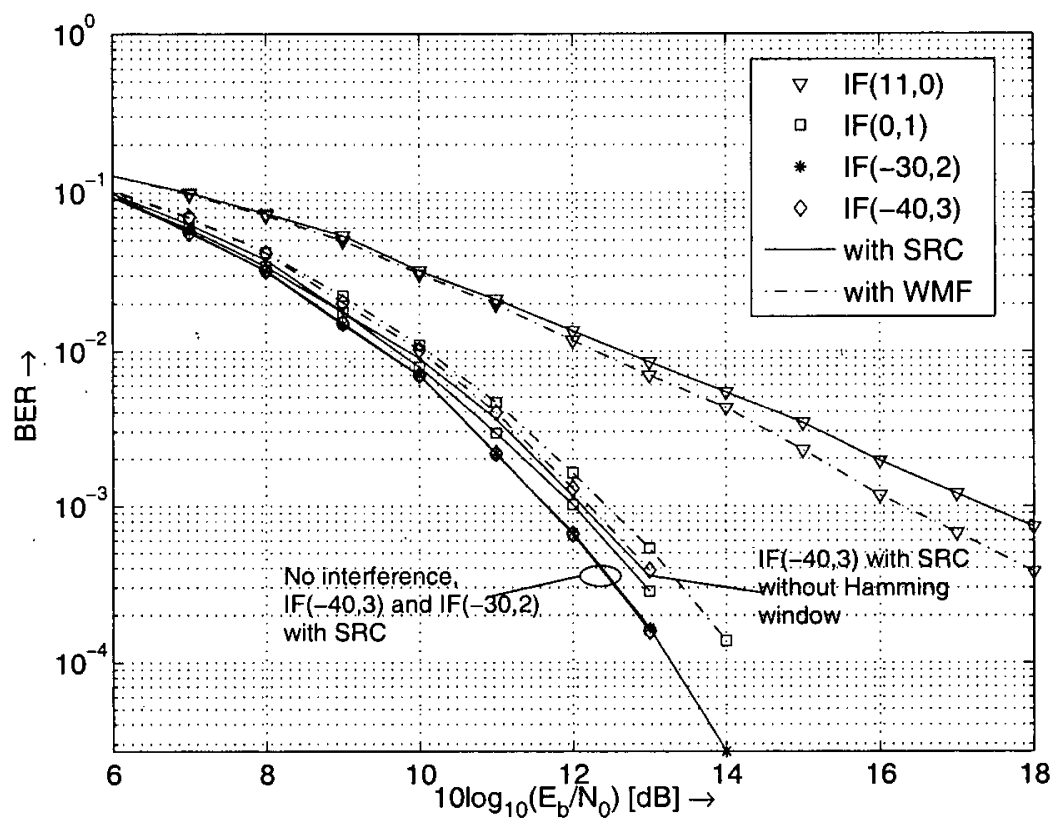

Figure 4.21: Performance of NDFE in the presence of interference for the WMF and the SRC filter. The channel phase is constant and the notation $\operatorname{IF}\left(\operatorname{SIR}, \Delta f_{c, i}\right)$ applies.

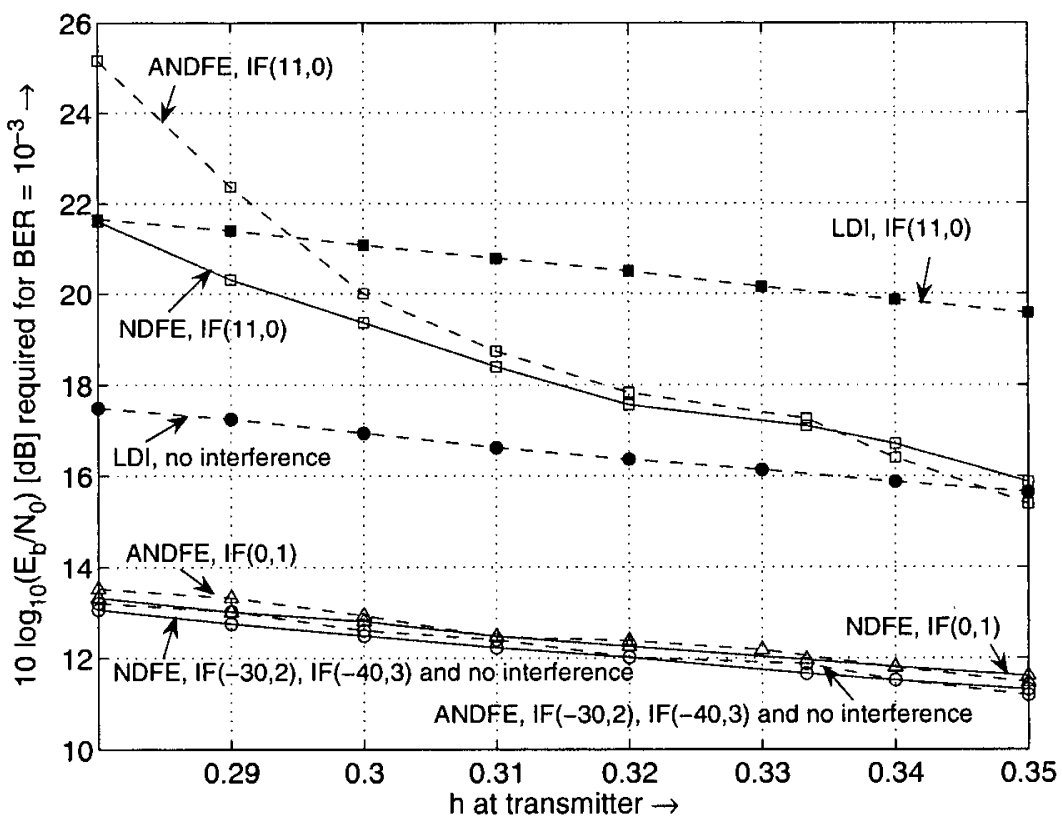

Figure 4.22: $10 \log _{10}\left(E_{b} / N_{0}\right)$ required for BER $=10^{-3}$ for NDFE and ANDFE in the presence of interference. The channel phase is constant and the notation $\operatorname{IF}\left(\operatorname{SIR}, \Delta f_{c, i}\right)$ applies. 
co-channel interference, an accountable performance loss of up to $3 \mathrm{~dB}$ over the NDFE is recorded for $h<0.3$. Also, ANDFE performs worse than the LDI for $h<0.295$. However, in practice, co-channel interference is a rare occurrence, and, adjacent channel interference is of greater interest in general. 


\section{Chapter 5}

\section{Conclusions}

The complexity constraints coupled with the varying modulation index and the high frequency offset variations in Bluetooth devices creates a highly challenging environment for the design of power-efficient Bluetooth receivers. The conventional LDI detector is a simple receiver, but is highly suboptimum in terms of power efficiency, and can not cope with the high frequency offsets of up to $\pm 100 \mathrm{KHz}$ admissible in Bluetooth systems. Several sequence detectors proposed in literature have proven to be extremely power efficient compared to the LDI detector since they properly take the CPM memory into account. These include the MLM-LDI and the MAP receivers. However, the MLM-LDI has very high complexity and the MAP receiver assumes perfect channel phase estimation and knowledge of the modulation index at the receiver, and, therefore, they are impractical. The NSD receiver recently proposed in [31] tackles these problems by designing a noncoherent sequence detector with a modulation index estimator. Although the complexity of the NSD is low compared to the MLM-LDI and the MAP receivers, it still requires a 2-state Viterbi decoder.

In this research work, we have presented a simple noncoherent power-efficient receiver design for Bluetooth transmission based on Laurent's decomposition, which transforms the actual nonlinear modulation scheme into a linear modulation over an intersymbol 
interference channel. It was shown that the combination of an SRC filter, symbolrate sampling, and a decision-feedback equalizer achieves a performance close to the theoretical MLSE limit. We have proposed a noncoherent version, namely NDFE, which offers high robustness to local-oscillator dynamics and phase jitter present in Bluetooth devices. NDFE was also extended to allow adaptation of the decision metric to an a priori unknown modulation index $h$. The presented simulation results showed that NDFE performs very similar to 2-state NSD proposed in [31]. The key advantages of the proposed design are outlined in the following and a summary of the corresponding simulation results presented in Chapter 4 is given.

- Using Laurent's decomposition, the optimal receiver input filter for the resulting PAM signal is the whitened matched filter (WMF) [17]. However, since the WMF is dependent on the modulation index $h$, we propose replacing it by the practical SRC filter which has similar characteristics. The simulations (cf. Fig. 4.1) prove that the resulting performance using the WMF and SRC are almost identical. Moreover, high adjacent channel interference suppression is achieved through the $\mathrm{SRC}$, as illustrated in Fig. 4.22.

- Following the SRC filter, a noncoherent decision feedback equalizer is employed, where the phase is implicitly estimated using the developed phase reference (3.46). This phase reference is formulated in terms of a forgetting factor $\alpha$ and shows remarkable performance improvement compared to the LDI, closely approaching the MLSD lower bound as $\alpha$ increases (cf. Fig. 4.6). However, since this phase reference fails in the presence of extreme frequency offset variations and phase jitter, a modified phase reference (3.53) is devised. Again, the modified phase reference is controlled by a forgetting factor $\beta$, and the resulting decision rule was found to be extremely robust against channel phase variations, as shown in Fig. 4.16. The pair ( $\alpha=0.6, \beta=0.9$ ) which was found to be favorable in NDFE for various scenarios, has almost the same power efficiency as the 2-state NSD 
using the same values of $\alpha$ and $\beta$.

- The NDFE is the simplest sequence detector possible, consisting of a one-state trellis, thus, providing an extremely high reduction in complexity compared to the $2 p$-state trellis required by the optimal detector. Only a slight performance loss was recorded by decreasing the trellis from a full-state to a one-state trellis (cf. Fig. 4.4). This is a remarkable result since the one-state trellis in the NSD receiver was shown to have worse performance than the low-complexity LDI detector [27].

- One of the primary disadvantages of the sequence detectors proposed in literature is assuming a nominal modulation index $h$ at the receiver. In the new receiver design, we propose employing an adaptive $h$-estimation scheme with an adaptation period of $N_{e}$ symbols, and a set $\mathcal{H}$ of hypothetical modulation indices. This adaptive version of NDFE, which does not require knowledge of the modulation index $h$, was found highly effective with $N_{e}=50$ and $|\mathcal{H}|=2$ or 4 , only adding a slight increase in complexity. The simulation results presented in Figs. 4.19-4.20 show that it performs almost identically to the case when $h$ is known, and very close to the adaptive version of NSD with the same values of $N_{e}$ and $|\mathcal{H}|$.

In conclusion, the new receiver design is highly attractive for a practical implementation, achieving a performance gain of more than $4 \mathrm{~dB}$ over the LDI detector with only a slight increase in complexity, and a performance loss of only about $2 \mathrm{~dB}$ compared to the MLSD lower bound with an extreme decrease in complexity. In addition, it achieves performance results similar to the NSD in various scenarios, inspite of its complexity advantage over NSD. Consequently, the NDFE receiver is favorable over all state-of-the-art Bluetooth receivers available in literature. For practical applicability, we suggest developing a cost-effective hardware implementation for NDFE. We believe that we have presented a complete framework in the present work, and, therefore, no additional theoretical analysis is required. 


\section{ก}

\section{Bibliography}

[1] The official Bluetooth website. http://www.bluetooth.com.

[2] J.B. Anderson, T. Aulin, and C.-E. Sundberg. Digital Phase Modulation. Plenum Press, New York, 1986.

[3] M.E. Austin. Decision-feedback equalization for digital communication over dispersive channels. Technical report 437, MIT Lincoln Laboratory, Lexington, MA, August 1967.

[4] L.R. Bahl, J. Cocke, F. Jelinek, and J. Raviv. Optimal decoding of linear codes for minimizing symbol error rate. IEEE Transactions on Information Theory, 20(2):284-287, March 1974.

[5] D.M. Bakker and D.M. Gilster. Bluetooth End to End. John Wiley \& Sons, Inc, 2002.

[6] O. Bar-Shalom, G. Chinn, K. Fleming, and U. Gadamsetty. On the union of WPAN and WLAN in mobile computers and hand-held devices. Intel Technology Journal, 7(3), August 2003.

[7] C.A. Belfiore and Jr. J.H. Park. Decision feedback equalization. Proceedings of the IEEE, 67(8):1143-1156, August 1979.

[8] E. Biglieri. Coding for Wireless Channels. Springer, 2005. 
[9] C. Bisdikian. An overview of the Bluetooth wireless technology. IEEE Communications Magazine, pages 86-94, December 2001.

[10] G. Colavolpe and R. Raheli. Reduced-complexity detection and phase synchronization of cpm signals. IEEE Transactions on Communications, 45(9):1070-1079, September 1997.

[11] G. Colavolpe and R. Raheli. Noncoherent sequence detection of continuous phase modulations. IEEE Transactions on Communications, 47(9):1303-1307, September 1999 .

[12] G. Colavolpe and R. Raheli. Detection of linear modulations in the presence of strong phase and frequency instabilities. IEEE Transactions on Communications, 50(10):1617-1626, October 2002.

[13] A.C. Davies. An overview of Bluetooth wireless technology and some competing LAN standards. In Proceedings of the IEEE International Conference on Circuits and Systems for Communications, pages 206-211, St.Petersburg, Russia, June 2002 .

[14] A. Demir, A. Mehrota, and J. Roychowdhury. Phase noise in oscillators: A unifying theory and numerical methods for characterization. IEEE Transactions on Circuits and Systems-I: Fundamental Theory and Applications, 47(5):655-674, May 2000 .

[15] A. Duel-Hallen and C. Heegard. Delayed decision-feedback sequence estimation. IEEE Transactions on Communications, 37(5):428-436, May 1989.

[16] M. V. Eyuboğlu and S.U.H. Quershi. Reduced-state sequence estimation with set partitioning and decision feedback. IEEE Transactions on Communications, 36(1):13-20, January 1988. 
[17] Jr. G.D. Forney. Maximum-likelihood sequence estimation of digital sequences in the presence of intersymbol interference. IEEE Transactions on Information Theory, 18(3):363-378, May 1972.

[18] Jr. G.D. Forney. The Viterbi algorithm. Proceedings of the IEEE, 61(3):268-278, May 1973.

[19] W. Gerstacker, R. Schober, and J. Huber. Noncoherent equalization algorithms based on sequence estimation. In Proceedings IEEE Global Conference on Communications, pages 3485-3490, Sydney, Australia, November 1998.

[20] W.H. Gerstacker, F. Obernosterer, R. Mayer, and J.B. Huber. On prefilter computation for reduced-state equalization. IEEE Transactions on Wireless Communications, 1(4):793-800, October 2002.

[21] J.C. Haarsten. Bluetooth- The universal radio interface for ad-hoc, wireless connectivity. Ericsson Review, Issue 3:110-117, 1998.

[22] J.C. Haartsen and S. Mattison. Bluetooth- A new low-power radio interface providing short-range connectivity. Proceedings of the IEEE, 88(10):1651-1661, October 2000 .

[23] X. Huang and Y. Li. The PAM decomposition of CPM signals with integer modulation index. IEEE Transactions on Communications, 51(4):543-546, April 2003.

[24] X. Huang and Y. Li. Simple noncoherent CPM receivers by PAM decomposition and MMSE equalization. In Proceedings IEEE International Symposium on Personal, Indoor, and Mobile Radio Communications, volume 1, pages 707-711, Beijing, China, September 2003.

[25] J.B. Huber and W. Liu. An alternative approach to reduced-complexity CPM receivers. IEEE Journal on Selected Areas in Communications, 7(9):1437-1449, December 1989. 
[26] IEEE documentation IEEE P802.15-01/444R1. IEEE P802.15 wireless personal area networks, October 2001.

[27] Mani Jain. Noncoherent sequence detection for Bluetooth systems. Master's thesis, University of British Columbia, September 2004.

[28] G.K. Kaleh. Simple coherent receivers for partial response continuous phase modulation. IEEE Journal on Selected Areas in Communications, 7(9):1427-1436, December 1989.

[29] S-C. Kim, H.L. Bertoni, and M. Stern. Pulse propagation characteristics at 2.4 GHz inside buildings. IEEE Transactions on Vehicular Technology, 45(3):579-592, August 1996.

[30] L. Lampe, R. Schober, G. Ezner, and J. Huber. Coded continuous phase modulation with low complexity noncoherent reception. IEEE Transactions on Communications, 50(4):517-520, April 2002.

[31] L. Lampe, R. Schober, and M. Jain. Noncoherent sequence detection receiver for Bluetooth systems. IEEE Journal on Selected Areas in Communications, 23(9):1718-1727, September 2005.

[32] P.A. Laurent. Exact and approximate construction of digital phase modulations by superposition of amplitude modulated pulses (AMP). IEEE Transactions on Communications, 34(2):150-160, February 1986.

[33] T.H. Lee and A. Hajimiri. Oscillator phase noise: A tutorial. IEEE Journal of Solid-state circuits, 35(3):326-336, March 2000.

[34] Patricia McDermott-Wells. What is Bluetooth? IEEE Potentials, 23(5):33-35, December 2004/ January 2005. 
[35] U. Mengali and M. Morelli. Decomposition of M-ary CPM signals into pam waveforms. IEEE Transactions on Information Theory, 41(5):1265-1275, September 1995

[36] P. Monsen. Feedback equalization for fading dispersive channels. IEEE Transactions on Information Theory, 17(1):56-64, January 1971.

[37] L. Lo Presti and M. Mondin. Design of optimal FIR rasied-cosine filters. Electronic letters, 25(7):467-467, March 1989.

[38] J.G. Proakis. Digital Communications. McGraw-Hill, fourth edition, 2000.

[39] R. Raheli, A. Polydoros, and C.-K. Tzou. Per-survivor processing: A general approach to MLSE in uncertain environments. IEEE Transactions on Communications, 43(2/3/4):354-364, Feb./Mar./Apr. 1995.

[40] T.S. Rappaport. Wireless Communications Principles and Practice. Prentice Hall, second edition, 2002.

[41] B.E. Rimoldi. A decomposition approach to CPM. IEEE Transactions on Information Theory, 34(2):260-270, March 1988.

[42] C. Robinson and A. Purvis. Demodulation of Bluetooth GFSK signals under carrier frequency error conditions. In Proceedings of the IEE Colloquium on DSPEnabled Radio, Scotland, UK, September 2003.

[43] R. Schirphorst, F.W. Hoeksema, and C.H. Slump. A (simplified) Bluetooth maximum a posteriori probability (MAP) receiver. In Proceedings of IEEE Workshop on Signal Processing Advances in Wireless Communications, pages 160-164, Rome, Italy, June 2003.

[44] R. Schober and W.H. Gerstacker. Metric for noncoherent sequence estimation. Electronic Letters, 35(25):2178-2179, December 1999. 
[45] R. Schober and W.H. Gerstacker. Adaptive noncoherent DFE for MDPSK signals transmitted over ISI channels. IEEE Transactions on Communications, 48(7):1128-1140, July 2000

[46] Robert Schober. Noncoherent detection and equalization for MDPSK and MDAPSK signals. Dissertation im Fachbereich Elektrotechnik, Universität Erlangen-Nürnberg, May 2000.

[47] T. Scholand and P. Jung. Least squares based post-integration filtering for robust digital LDI receivers. In Proceedings of IEEE International Symposium on Signal Processing and Information Technology, pages 818-821, New York, USA, December 2003.

[48] T. Scholand and P. Jung. Novel receiver structure for Bluetooth based on modified zero-crossing demodulation. In Proceedings of IEEE Global Telecommunications Conference, volume 2, pages 729-733, San Fransisco, USA, December 2003.

[49] T. Scholand, A. Waadt, T. Faber, and P. Jung. Maximum-likelihood symbol-bysymbol postprocessing applied to digital limiter-discriminator-integrator based IF detectors. In Proceedings of IEEE Vehicular Technology Conference, volume 6, pages 3838-3842, Los Angeles, USA, September 2004.

[50] T. Scholand, A. Waadt, and P. Jung. Max-log-ML symbol estimation postprocessor for intermediate frequency LDI detectors. Electronics Letters, 40:183-185, February 2004.

[51] Bluetooth Special Interest Group (SIG). Bluetooth standard core version $2.0+$ EDR, November 2004.

[52] M.K. Simon and C.C. Wang. Differential detection of Gaussian MSK in a mobile radio environment. IEEE Transactions on Vehicular Technology, 33(4):307-320, November 1984. 
[53] A. Soltanian and R.E. Van Dyck. Performance of the Bluetooth system in fading dispersive channels and interference. In Proceedings of the IEEE Global Telecommunications Conference, volume 6, pages 3499-3503, San Antonio, TX, November 2001.

[54] A. Soltanian and R.E. Van Dyck. Physical layer performance for coexistance of Bluetooth and IEEE 802.11b. In Proceedings of Virginia Technology Symposium on Wireless Personal Communications, Blacksburg, VA, June 2001.

[55] A. Viterbi. Error bounds for convolutional codes and an asymptotically optimum decoding algorithm. IEEE Transactions on Information Theory, 13(2):260-269, April 1967.

[56] K.C. Yu and A.J. Goldsmith. Linear models and capacity bounds for continuous phase modulation. In Proceedings of IEEE International Conference on Communications, volume 2, pages 722-726, New York City, USA, April 2002. 